I N T ER N ATIONAL MONETARY FUND

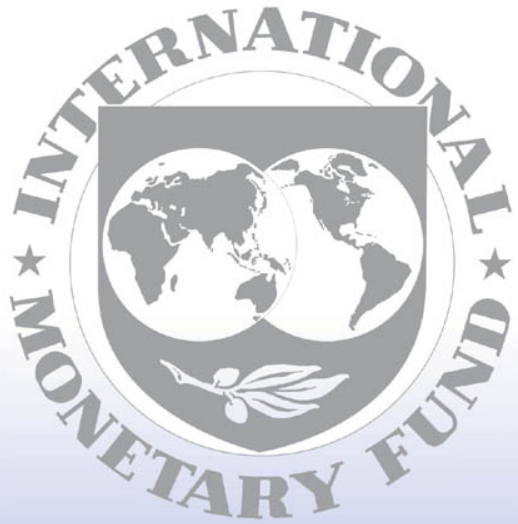

Staff

Country

Reports 


\title{
Democratic Republic of Timor-Leste: 2010 Article IV Consultation-Staff Report; Joint World Bank/IMF Debt Sustainability Analysis; Staff Statement; Public Information Notice on the Executive Board Discussion; and Statement by the Executive Director for Timor-Leste
}

Under Article IV of the IMF's Articles of Agreement, the IMF holds bilateral discussions with members, usually every year. In the context of the 2010 Article IV consultation with the Democratic Republic of Timor-Leste, the following documents have been released and are included in this package:

- The staff report for the 2010 Article IV consultation, prepared by a staff team of the IMF, following discussions that ended on November 9, 2010, with the officials of the Democratic Republic of Timor-Leste on economic developments and policies. Based on information available at the time of these discussions, the staff report was completed on December 3, 2010. The views expressed in the staff report are those of the staff team and do not necessarily reflect the views of the Executive Board of the IMF.

- $\quad$ A supplement consisting of the joint World Bank/IMF debt sustainability analysis.

- $\quad$ A staff statement of January 19, 2011, updating information on recent developments.

A Public Information Notice (PIN) summarizing the views of the Executive Board as expressed during its January 28, 2011 discussion of the staff report that concluded the Article IV consultation.

- $\quad$ A statement by the Executive Director for the Democratic Republic of Timor-Leste.

The policy of publication of staff reports and other documents allows for the deletion of market-sensitive information.

Copies of this report are available to the public from

International Monetary Fund $\bullet$ Publication Services

$70019^{\text {th }}$ Street, N.W. $\bullet$ Washington, D.C. 20431

Telephone: (202) 623-7430 • Telefax: (202) 623-7201

E-mail: publications@imf.org Internet: http://www.imf.org

\author{
International Monetary Fund \\ Washington, D.C.
}




\section{INTERNATIONAL MONETARY FUND}

\section{DEMOCRATIC REPUBLIC OF TIMOR-LESTE}

\section{Staff Report for the 2010 Article IV Consultation ${ }^{1}$}

Prepared by Staff Representatives for the 2010 Consultation with the Democratic Republic of Timor-Leste

Approved by Ray Brooks and Dhaneshwar Ghura

December 3, 2010

\section{Key Issues and Recommendations:}

- Context: Petroleum-financed government spending supported double-digit growth in 200709 and commendable progress was made in reducing poverty.

- Growth prospects: in 2010 non-oil GDP growth is expected to slow to around 6 percent due to the impact of adverse weather on harvests. Scaled up public investment will be a key driver of growth in the medium-run, though there is uncertainty about the size, timing, and quality of capital spending.

- Focus: Discussions focused on how to manage the petroleum wealth to develop the non-oil economy.

- Appropriate pace of public spending growth: The government plans to fast-track development by ratcheting up capital spending rapidly. The mission supported wellmanaged infrastructure investment but suggested a more-gradual-than-planned pace of growth in capital spending while lowering capacity constraints.

- External stability: Officially dollarized, competitiveness depends on business-enabling reforms, raising labor skills, and a slower pace of government spending to contain inflationary pressures.

\footnotetext{
${ }^{1}$ The report is based on discussions during October 26-November 9, 2010, with Prime Minister Gusmao, Finance Minister Pires, General Manager of the Banking and Payments Authority Vasconcelos, State Secretaries of agriculture, commerce and tourism, and other senior officials, and representatives of donors, the business community, and civil society. The team comprised Messrs. Schule (head), Ochirkhuu, Ms. Sun (all APD), Mr. Cardoso (OED), Messrs. Rab, Suri (both World Bank), and Kang (Asian Development Bank).
} 


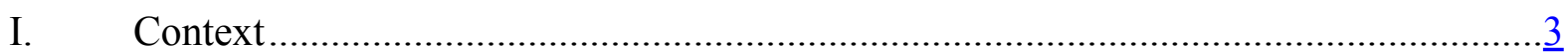

II. Recent Developments ....................................................................................

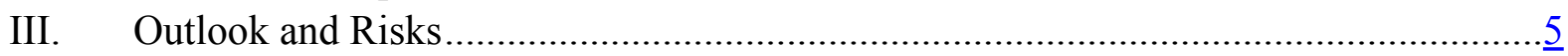

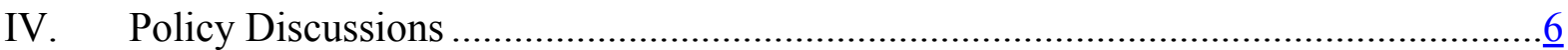

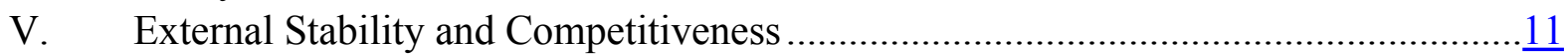

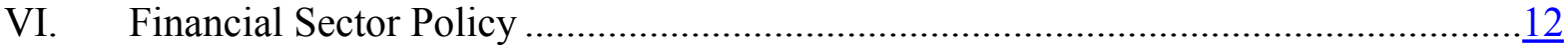

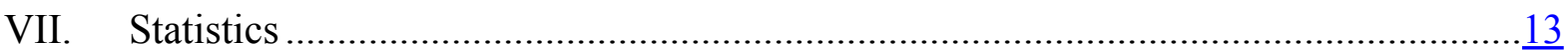

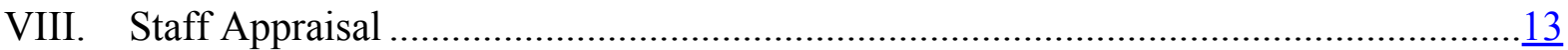

Box

1. Exchange Rate, Competitiveness, and External Sustainability Assessment..................15

Figures

1. Regional and Global Comparisons …………………..........................................16

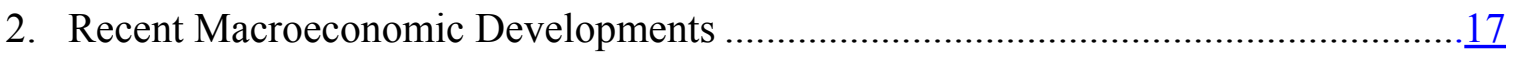

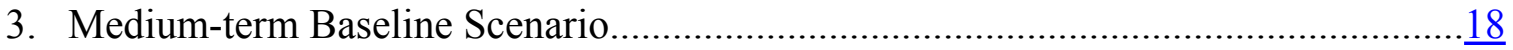

Tables

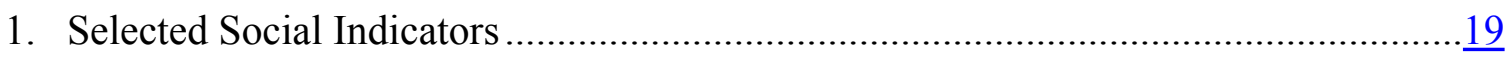

2. Selected Social and Economic Indicators ……….....................................................

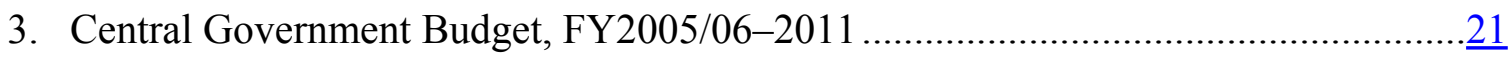

4. Combined Sources Budget, 2004-2011 ………….....................................................

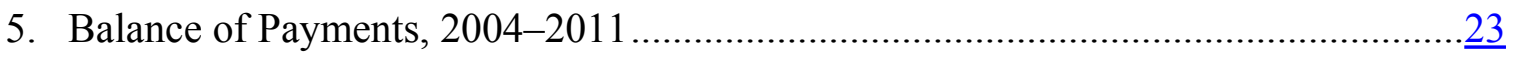

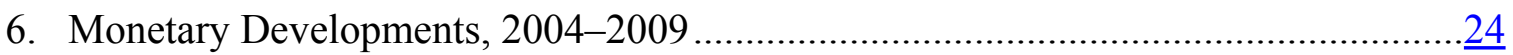

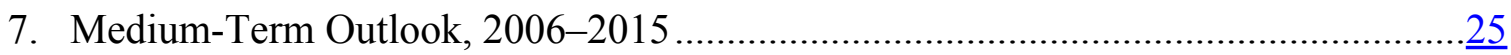

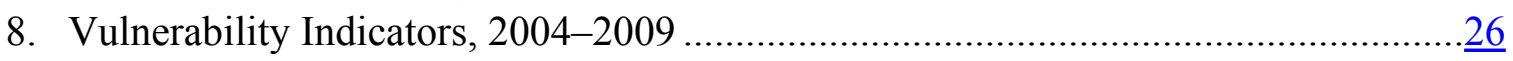

9. Millennium Development Goals Progress, 1990-2008 …………………………......27

Appendix Table

1. Authorities' Response to Recent Fund Policy Advice................................................

Annexes

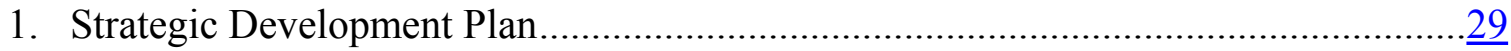

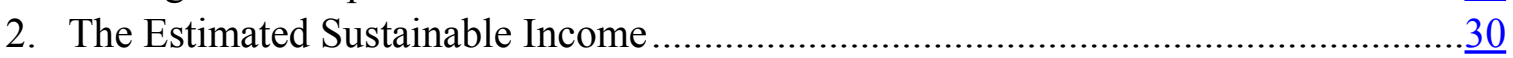

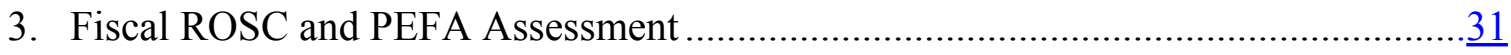

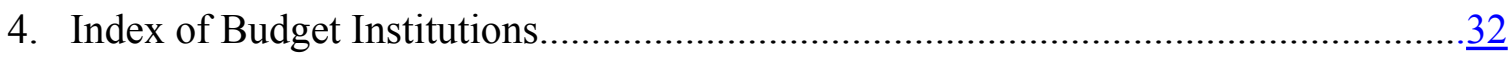




\section{ConteXt}

\section{In its first eight years as an independent nation, Timor-Leste has made} commendable economic progress, though it still faces many development challenges. About 40 percent of the population still lives in poverty and the 2010 Human Development Report (based on 2009/10 data) ranked Timor-Leste at 120 out of a total of 169 countries (Figure 1). Years of conflict have destroyed a large part of its infrastructure. However, the country is blessed with natural resources, with offshore oil production starting in 2004. Although its oil wealth is currently estimated at $\$ 24.3$ billion or $\$ 22,000$ per capita, and more reserves such as the Greater Sunrise have been discovered, it is neither a major oil producer nor an upper middle income country. It stands out as the most oil dependent country globally. In 2009, petroleum income accounted for about 95 percent of total government revenue and almost 80 percent of GNI.

\section{An overarching challenge for Timor-Leste is to manage petroleum wealth} effectively to raise growth and living standards on a sustainable basis. The 2010 Article IV Consultations took place against a backdrop of the authorities' plans to fast track development by scaling up public investment in infrastructure and human capital. The government has outlined its strategy in a summary of the Strategic Development Plan (SDP) for 2011-2030 (Annex 1). The authorities have been generally responsive to the Fund's past policy advice (Appendix Table 1).

\section{RECENT DEVELOPMENTS}

3. The past three years saw rapid economic growth and reduced poverty. Improved security following the 2006 civil unrest, higher oil-financed public spending, and a rebound in agriculture from the 2007 drought have contributed to non-oil GDP growth averaging 11 percent during 2007-09 (Figure 2). Staff expects non-oil GDP growth to drop to about 6 percent in 2010, largely reflecting the detrimental impact of unseasonal rainfall and floods on agricultural production. Foreign direct investment increased, mainly in the petroleum sector, but private investment remains modest at about 10 percent of non-oil GDP. A recent estimate by the World Bank shows a decline of poverty incidence from 50 percent in 2007 to

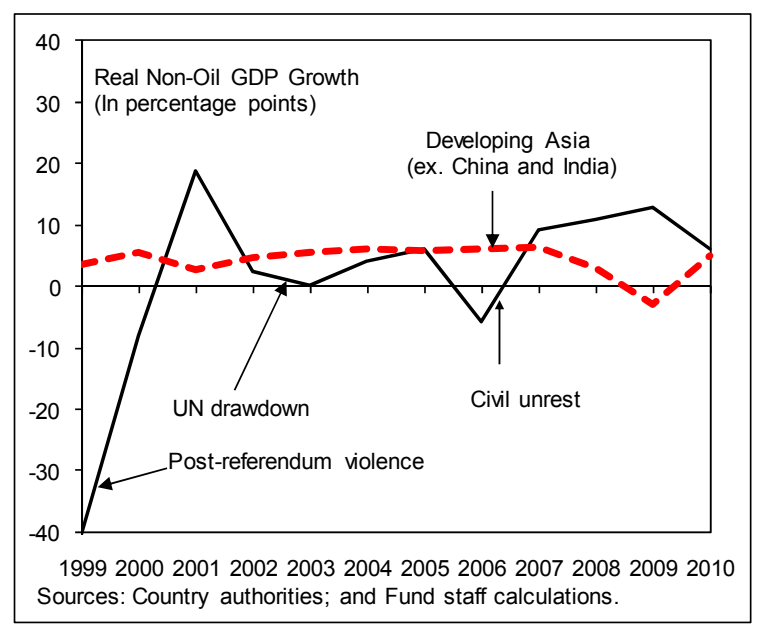
41 percent in 2009 .

4. Inflation largely reflected movements in international food and energy prices. Annual CPI inflation fell sharply from 12 percent in mid-2008 to just 1 percent at end-2009, and has since risen to 5.3 percent year on year in the September quarter of 2010. Domestic 
price pressures have been rising and are particularly high in some segments of the economy, such as housing and construction, and in the capital Dili where annual inflation reached 7.5 percent in September 2010. In each of the past two years, wages in the public sector increased by 25 percent and, according to a provisional survey by the Ministry of Finance, 9 percent in the private sector in 2010.

\section{The fiscal position strengthened in 2009 .}

Following a 130 percent increase in 2008 , public

spending grew by a more moderate rate of 8 percent to $\$ 604$ million in 2009 , with capital expenditure accounting for most of the increase. As a result, the non-oil fiscal deficit fell by 17 percentage points to 92 percent of non-oil GDP. For the first time, the deficit was financed by $\$ 512$ million withdrawals from the petroleum fund (PF) in excess of the $\$ 408$ million certified estimated sustainable income from petroleum (ESI). The ESI guides fiscal policy as to the amount that can be withdrawn from the PF without depleting it over time (Annex 2).

\section{The original 2010 budget foresaw continued moderate spending growth.}

However, in June 2010, the authorities introduced a supplemental budget that enlarged this year's spending envelop to $\$ 838$ million, an increase of almost 40 percent over actual spending in 2009, largely financed by withdrawals of $\$ 811$ million from the PF. But given procurement delays, particularly for capital spending, staff suggest that 2010 cash spending will be around $\$ 650$ million, well below the revised spending envelope.

\section{The current account surplus in percent of non-oil GDP declined by almost half to} 245 percent in 2009. Lower international oil prices reduced oil earnings, recorded as income on the balance of payments. Non-oil exports such as coffee and tourism have remained insignificant compared to oil. Merchandise imports grew by 24 percent, reflecting higher public spending and the economy's limited absorptive capacity.

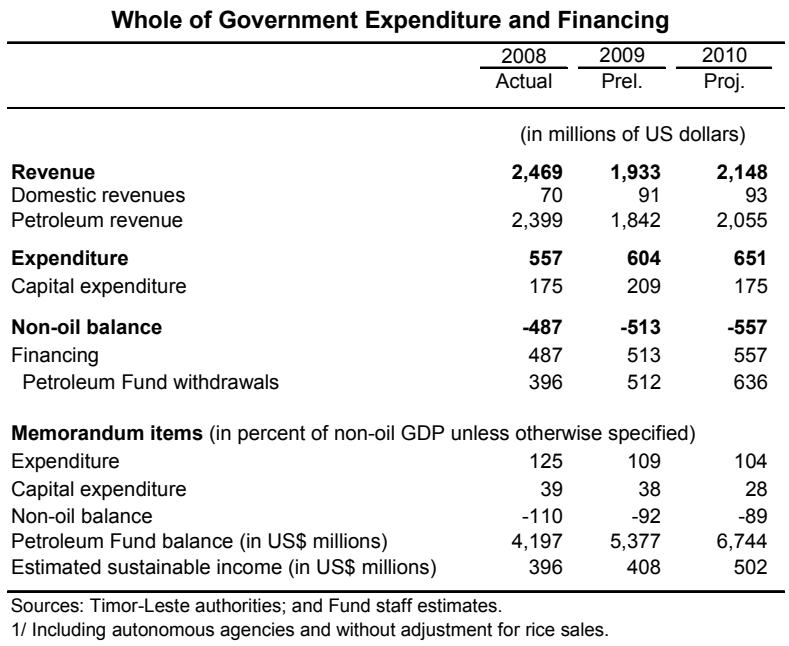

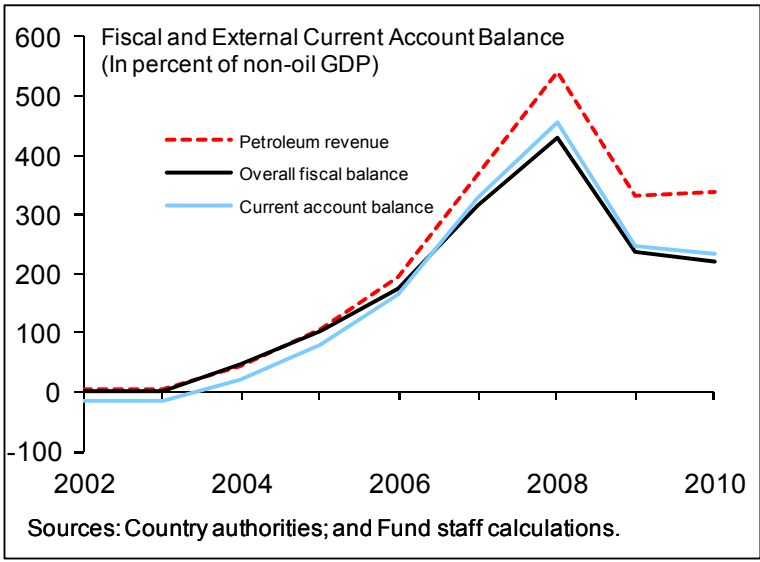


8. The PF assets have accumulated substantially over the past 5 years to reach \$6.5 billion or about ten times non-oil GDP at end-August 2010. Owing to lower international oil prices, petroleum-related revenue declined in 2009 , but is expected to recover to $\$ 2.1$ billion in 2010 . As a result, the overall fiscal surplus to non-oil GDP ratio is projected to decline from 430 percent in 2009 to 240 percent in 2010.
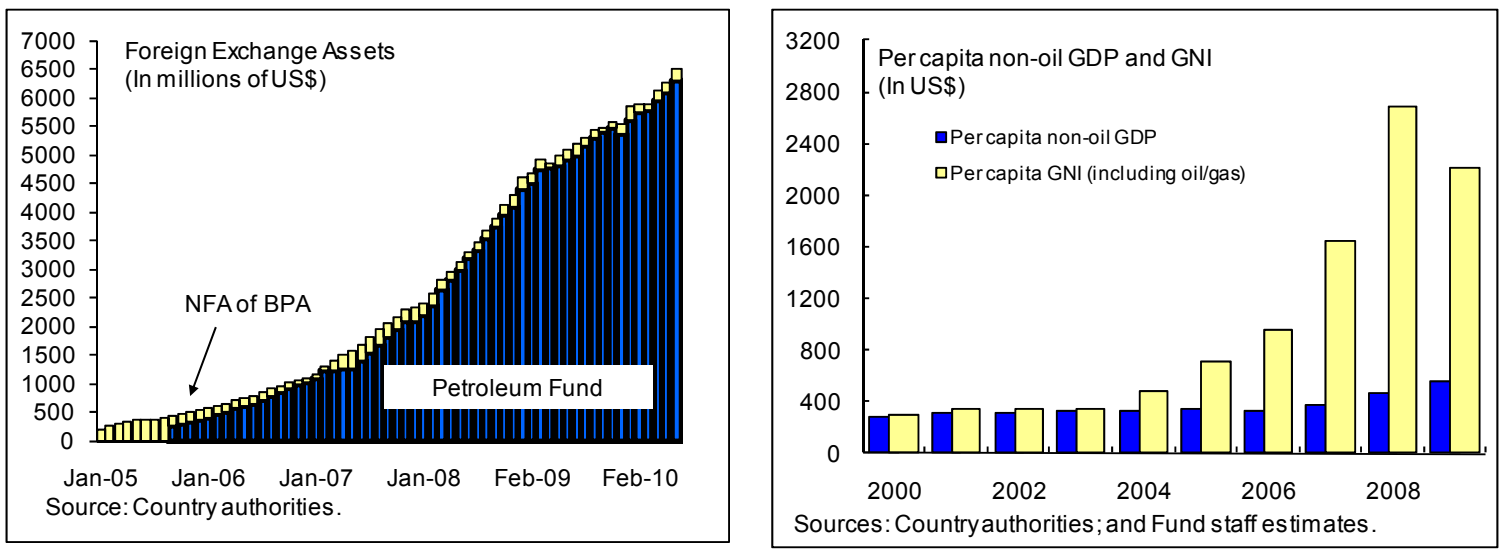

\section{OUTLOOK AND RISKS}

9. The growth outlook remains positive, supported by social and political stability and increased government spending.

- Near term. GDP growth is expected to increase to around $71 / 3$ percent in 2011 , driven by government spending, which is projected to increase by almost 40 percent in 2011 . Activity in private construction and commerce is also expected to be dynamic, while the recovery in agriculture remains gradual. Inflation is expected to average $4 \frac{1}{2}$ percent in 2010, and to increase to 6 percent in 2011 .

- Medium term. Scaled-up public investment will remain a key driver of growth in the medium term, although its effectiveness may be limited by Timor-Leste's weak public financial management capacity and a disproportionally small size of the private sector. As a result, annual non-oil growth is expected to re-accelerate in 2012 and to peak at 93/4 percent in 2013 (Figure 3).

- Long term. Non-oil growth is to gradually converge to around 6 percent in the long run, when the private sector will have to become the main driver of growth.

10. However, large uncertainties surround the outlook. Oil revenues are sensitive to volatile international oil prices and to interruptions in production. Domestically, there is uncertainty about the size, timing, and quality of capital spending, especially for the medium term. Moreover, the depth and pace of business-enabling reforms is difficult to predict. 


\section{Authorities' Views}

11. The authorities shared the mission's positive assessment of the economy, but expected double-digit growth rates. They projected a higher growth impact of public investment and more private sector growth and noted that staff's 2008-09 growth projections were consistently below actual outturns. For 2010, the authorities agreed that the economy would slow down, but could still approach a double-digit growth rate. The authorities projected the economy to continue recording double-digit growth in the medium and long run, with improving budget planning and execution. There was an agreement that any projections are surrounded by large uncertainties given data constraints and a rapid change in the structure of the non-oil economy. The authorities suggested that staff provide upper and lower bounds of projections in the future pursuant to the conditions of Timor-Leste.

\section{Policy Discussions}

\section{A. Fiscal Policy}

\section{The Pace of Public Spending Growth}

12. Pressing development needs call for more and better public spending. Petroleum wealth provides a unique opportunity to transform Timor-Leste into a higher middle income country. Following rapid growth in recent years, the level of public expenditure per capita exceeds that of many other petroleum-exporting LICs. A key challenge going forward is to enhance the effectiveness of public spending and address supply and structural constraints in the non-oil economy. The SDP is a positive step toward defining a growth and povertyreduction strategy. However, concrete implementation plans remain to be worked out and projects need to be costed and
Public Expenditure ratios in low and middle income countries

\begin{tabular}{lrrrrr}
\multicolumn{5}{c}{ Countries 1/ } & \multicolumn{4}{c}{ Per capita primary spending (US\$) } \\
\cline { 2 - 3 } \cline { 5 - 6 } & 2000 & 2007 & 2008 & 2009 & 2010 \\
\hline \hline Congo DR (USD 171) & 6 & 26 & 38 & 41 & 61 \\
Yemen, Republic of (USD 1061) & 46 & 55 & 56 & 41 & 45 \\
Cameroon ( USD 1114) & 84 & 165 & 222 & 202 & 205 \\
Sudan (USD 1398) & 39 & 313 & 340 & 271 & 333 \\
Vietnam (USD 1068) & 96 & 223 & 278 & 341 & 342 \\
Indonesia (USD 2330) & 105 & 339 & 416 & 362 & 466 \\
Timor-Leste (USD 543) & $\mathbf{5 3}$ & $\mathbf{2 3 1}$ & $\mathbf{5 1 5}$ & $\mathbf{5 4 2}$ & $\mathbf{5 6 6}$ \\
Colombia (USD 5167) & 563 & 1158 & 1211 & 1345 & 1555 \\
Azerbaijan, Rep. of (USD 4807) & 132 & 971 & 1618 & 1664 & 1834 \\
Kazakhstan (USD 7019) & - & 1613 & 2302 & 1713 & 2212 \\
Gabon (USD 7468) & 662 & 1510 & 1844 & 1744 & 1814 \\
Algeria (USD 3996) & 443 & 1271 & 1856 & 1646 & 2144 \\
Equatorial Guinea (USD 9580) & 273 & 1978 & 3208 & 4688 & 2977 \\
Libya (USD 9511) & 2034 & 3850 & 5697 & 5259 & 5534 \\
Bahrain (USD 19817) & 2798 & 6708 & 7682 & 6007 & 5902 \\
\hline
\end{tabular}

1/ All countries are net petroleum exporters for which data are available. 2009 GDP per capita in parenthesis. prioritized. The timing of large infrastructure projects should take account of Timor-Leste's institutional weaknesses, administrative capacity constraints, and limited absorptive capacity of the economy, in particular due to the shortage of skilled labor and managerial capacity.

\section{The government's fiscal projections foresee significant and front-loaded scaling up of public investment and moderate growth in current spending.}

- $\quad$ For 2011, the draft budget presents an envelope of $\$ 985$ million, an increase of over 50 percent from the spending expected by staff in 2010 . However, such a large 
increase may be challenging to execute and staff expect cash expenditure in 2011 to grow at a slower pace and reach about $\$ 900$ million. The draft budget foresees current spending to grow moderately at 4 percent without taking account of the recurrent cost associated with public investment. The government plans to limit withdrawals from the PF to the 2011 ESI of $\$ 734$ million. The remaining financing will come from nonoil domestic revenue and the government's cash balance that has built up due to under-execution of the budget in 2010.

- In 2012-13, the authorities plan to increase public investment at an average rate of about 30 percent, and to maintain this level in 2014-15. Without taking account of the recurrent cost of capital spending, the government projects current spending to grow by only 4 percent per year over 2012-15. Staff projects that the government's spending plans, if largely implemented, will require withdrawals from the PF exceeding the ESI by an annual average of about $\$ 300$ million. Staff expects a significantly reduced overall fiscal surplus of 107 percent of non-oil GDP in 2015, while the non-oil fiscal deficit averages 108 percent of non-oil GDP during 2012-15.

\section{Staff recommended a more moderate increase in spending, accompanied by capacity building. Specifically:}

- Staff supports government investment in public infrastructure, health and education, if such spending has a sufficiently high rate of return, is well managed and efficiently and transparently implemented. Such investment would raise economic growth and the standard of living of Timorese people.

- $\quad$ However, present spending levels are stretching administrative capacities and creating bottlenecks in the economy. Outsourcing can help ease administrative capacity constraints, and staff encouraged the authorities to take full advantage of the expertise provided by the World Bank, ADB, and bilateral donors. Yet, managing outsourcing is a challenge in itself.

- $\quad$ The ESI continues to serve as guidance for public spending that should anchor fiscal policy over the medium term. The PF framework does not stand in the way of financing worthy investment projects even if that implies temporarily spending in excess of the ESI. Moreover, the move from using the low oil price forecast published by the U.S. Energy Information Administration (EIA) to using the average of EIA low and reference case oil price ${ }^{1}$ has raised the ESI from $\$ 502$ million in 2010 to $\$ 734$ million in 2011. The sum of domestic revenues and higher ESI, projected at $\$ 835$ million in 2011, forms a sustainable spending envelope for anchoring fiscal policy. Specifically, given the need to build up capacity, staff advised an average

\footnotetext{
${ }^{1}$ This mid-price is still some $\$ 15$ below market futures and can be considered prudent, as required by the PF law. See The Estimated Sustainable Income Calculation and Selected Petroleum Fiscal Issues, IMF TA report September 2010.
} 
spending growth of around 10 percent annually over the medium term. This spending path would entail that withdrawals from the PF are in line with the ESI levels on average (Annex 2) over the medium term, with withdrawals from the PF below the ESI in early years and above the ESI in later years.

\begin{tabular}{|c|c|c|c|c|c|c|c|c|c|}
\hline \multicolumn{10}{|c|}{ Timor-Leste: Medium-Term Fiscal Outlook } \\
\hline & \multicolumn{9}{|c|}{ Baseline Scenario } \\
\hline & 2008 & 2009 & 2010 & 2011 & 2012 & 2013 & 2014 & 2015 & $2011-152 /$ \\
\hline & Act. & Prel. & Proj. & Proj. & Proj. & Proj. & Proj. & Proj. & Proj. \\
\hline Real non-oil GDP growth (percent) & 11.0 & 12.9 & 6.1 & 7.3 & 8.6 & 9.7 & 9.4 & 8.2 & 8.7 \\
\hline Expenditure of the whole government (US\$ millions) $1 /$ & 557 & 604 & 651 & 898 & 1062 & 1205 & 1278 & 1271 & 1143 \\
\hline Non-oil balance of the whole government (US\$ millions) & -487 & -513 & -557 & -798 & -946 & -1075 & -1133 & -1109 & -1012 \\
\hline in percent of non-oil GDP & -110 & -92 & -89 & -113 & -117 & -116 & -106 & -91 & -109 \\
\hline Withdraw from the PF (US\$ millions) & 396 & 512 & 636 & 734 & 929 & 1045 & 1091 & 1054 & 971 \\
\hline Change of government's cash balance (US\$ millions) & -4 & 8 & 79 & -64 & 0 & 0 & 0 & 0 & -13 \\
\hline Loan disbursement (US\$ millions) & - & - & - & 0 & 17 & 30 & 42 & 55 & 29 \\
\hline Estimated sustainable income (US\$ millions) & 396 & 408 & 502 & 734 & 744 & 745 & 743 & 740 & 741 \\
\hline \multirow[t]{4}{*}{ Petroleum Fund assets (US\$ millions) } & 4,197 & 5,377 & 6,744 & 8,271 & 9,735 & 10,834 & 12,176 & 13,524 & 13,524 \\
\hline & \multicolumn{9}{|c|}{ Alternative Scenario } \\
\hline & 2008 & 2009 & 2010 & 2011 & 2012 & 2013 & 2014 & 2015 & $2011-15 / 2$ \\
\hline & Act. & Prel. & Proj. & Proj. & Proj. & Proj. & Proj. & Proj. & Proj. \\
\hline Real non-oil GDP growth (percent) & 11.0 & 12.9 & 6.1 & 6.1 & 6.6 & 7.4 & 8.2 & 8.9 & 7.4 \\
\hline Expenditure of the whole government (US\$ millions) $1 /$ & 557 & 604 & 651 & 724 & 800 & 892 & 985 & 1,088 & 898 \\
\hline Non-oil balance of the whole government (US\$ millions) & -487 & -513 & -557 & -625 & -687 & -762 & -833 & -910 & -763 \\
\hline in percent of non-oil GDP & -110 & -92 & -89 & -91 & -91 & -91 & -89 & -86 & -89 \\
\hline Withdraw from the PF (US\$ millions) & 396 & 512 & 636 & 624 & 669 & 732 & 791 & 855 & 734 \\
\hline Change of government's cash balance (US\$ millions) & -4 & 8 & 79 & - & - & - & - & - & - \\
\hline Loan disbursement (US\$ millions) & - & - & - & 0 & 17 & 30 & 42 & 55 & 29 \\
\hline Estimated sustainable income (US\$ millions) & 396 & 408 & 502 & 734 & 747 & 757 & 765 & 771 & 755 \\
\hline Petroleum Fund assets (US\$ millions) & 4,197 & 5,377 & 6,744 & 8,383 & 10,116 & 11,551 & 13,227 & 14,819 & 14,819 \\
\hline
\end{tabular}

Sources: Fund staff estimates.

$1 /$ Without adjustment for rice sales.

2/ PF assets end of 2015.

\section{The key advantage of the staff's alternative strategy is balanced and sustainable}

economic growth. A more gradual increase in public investment-accompanied by

strengthening public financial management and accelerating reforms to improve the business environment-would raise the efficiency of public investment and likely crowd-in private investment. While GDP growth may be slightly lower than in the baseline in the short run, economic development would become more balanced, with a stronger role of the private sector, and yield higher long-run growth rates. Spending would be more aligned with the absorptive capacity of Timor-Leste's economy, exert less inflationary pressures, and therefore reduce the risk of Dutch disease. The recommended spending path would also maintain fiscal sustainability without reliance on oil revenues from Greater Sunrise.

Moreover, a binding medium-term budget constraint would help improve the budget process by providing incentives for cost control, prioritization and better planning.

16. The composition of public spending warrants close examination. The implications of higher public investment for recurrent spending need to be carefully considered to ensure that the scaling-up of public investment does not jeopardize fiscal sustainability. Furthermore, the composition of public expenditure should take account of both economic and social objectives. Within current spending, there is room to restrain wage growth, phase out subsidies, including on rice and electricity, and to better target transfers to the most needy.

\section{The sustainability of public spending will also depend on the government's} ability to realize the fiscal dividends of growth. Compliance with tax obligations is weak, 
despite low statutory tax rates- 10 percent on income, and $2 \frac{1}{2}$ percent customs duty and sales tax. As a result, in 2009 the domestic tax to non-oil GDP ratio was only 8 percent. The authorities are aware that tax collection is ineffective outside the petroleum sector. Staff welcomed the governments' ongoing efforts to strengthen the tax administration, including by improving taxpayer registration, following through on non-compliance, and building tax audit capacity. However, should these measures prove insufficient to reach a short-term revenue target of \$200 million (about 25 percent of non-oil GDP in 2012), higher tax rates, and new taxes, including on land, should be considered. Conversely, new tax breaks in the draft Investment Law go in the wrong direction and may prove ineffective in raising private sector investment.

\section{The government is considering borrowing to finance large infrastructure}

projects. Current development plans could be exclusively financed by withdrawals from the $\mathrm{PF}$. However, development partners may offer financing at concessional terms or at interest rates below expected returns on PF assets, which could increase from the historical annual return of $4 \frac{1}{2}$ percent with the planned change of PF investment strategy. Importantly, such borrowing may come with the transfer of knowledge from these institutions. The mission welcomed the authorities' intention to channel borrowing through the budget subject to parliament approval, and suggested that any borrowing should substitute for withdrawals from the PF. Staff cautioned against borrowing from international financial markets.

\section{A first joint World Bank-IMF debt sustainability analysis (DSA) found that} Timor-Leste is at low risk of debt distress. A key assumption is that Timor-Leste would resort to limited borrowings from development partners over the next 20 years, which carry an average interest rate of not more than $4 \frac{1}{2}$ percent. The DSA also highlights Timor-Leste's sensitivity to petroleum price and production shocks. Therefore, to minimize the risk of debt distress, it is advisable for Timor-Leste to take a gradual and moderate approach to borrowing from development partners and be fiscally prudent.

\section{Public Financial Management}

\section{Staff commended the authorities' ongoing efforts to improve the public financial} management (PFM) system and their resolve to address remaining weaknesses. The recent fiscal ROSC and PEFA missions found solid progress albeit from a low base.

Nevertheless, despite of the government's ongoing efforts, significant gaps remain in PFM, notably little project assessment capacity, in particular on the capital side, weak planning and implementation capacity in line ministries, a haphazard procurement process, and an absence of internal audits (Annexes 3 and 4). The draft SDP is a welcome first step, but further work is needed to improve medium-term budgeting and planning. Reforms should be prioritized and consolidated while line ministries should participate fully in the PFM reform agenda. The Ministry of Finance is taking steps in this direction through the development of a Strategic Plan for PFM. 
21. The PF remains the cornerstone for managing the country's oil-based financial resources. The ESI has proven to be a useful benchmark to inform fiscal policy and public discussion, while the PF framework is flexible enough to allow for spending above the ESI in the long-term interest of Timor-Leste, subject to parliament approval. Staff welcomed that planned revisions to the PF Law leave its basic principles - transparency, accountability, and inter-generational equity - intact. However, some specifics as to what "justifies" withdrawals from the PF in excess of the ESI, such as financial or social returns, would be helpful to focus the public debate. Also, ambiguity as to who will be the manager of the PF is likely to create uncertainty.

\section{Staff encouraged the authorities to address under-spending problems, in particular regarding multi-annual capital projects and capacity building.}

- $\quad$ The first best approach would be to undertake comprehensive budget modernization to enhance budget credibility, both in terms of quantity and quality, in line with the recommendations of the Fund's fiscal ROSC, PEFA, and treasury management missions.

- $\quad$ The second option, adopted by the government, of creating special investment funds can be effective, if adequate yet flexible controls are established to prevent the funds from taking on undesirable characteristics of extra-budgetary funds. Therefore, staff welcomed the government's commitment to allocate money into these funds through the same budget rules as all other expenditures; to appropriate the use of fund resources to an identified entity and project; and to link non-lapsing provisions to a list of projects that have been approved through the normal budget process. The government is also committed to keep resources in the treasury single account and use the IFMIS system for the funds.

\section{The authorities' efforts to improve cost and quality control and combat} corruption are commendable. The planned centralization of project evaluation and approval in a new agency, which will report directly to the Prime Minister and be staffed by well-qualified professionals, can help address the lack of administrative assessment capacity and ensure more effective cost and quality control. At the same time, greater oversight and transparency of project approval and monitoring could help reduce corruption.

\section{Authorities' Views}

24. The authorities emphasized the need to scale up public investment quickly. They noted that many years of conflict have left the country's infrastructure in shambles. Given the country's stage of development, the state has to play a leading role in providing basic infrastructure. Rehabilitating existing and building new roads, providing access to reliable electrical power and clean water are seen by the authorities as a prerequisite to attract private investment. The government is conscious of many difficulties and has been working 
tirelessly to overcome capacity constraints. It has identified main investment priority areas, in line with the Strategic Development Plan, and felt the urgency to act without delay in order not to lose another generation.

\section{The authorities felt that front-loaded scaling up of public investment would be} consistent with long-term fiscal sustainability. Their position was that such spending serves the long-run interest of the country even though it requires withdrawals from the PF in excess of the ESI in the short run.

- Ultimately, a vital private sector will need to become the source of jobs, income, and fiscal revenue. However, without an essential public infrastructure in place, no such economy is likely to emerge.

- $\quad$ Once basic infrastructure is in place, large public spending can be reduced and withdrawals from the PF can return to sustainable levels.

- $\quad$ The current ESI does not capture potential revenues from the Greater Sunrise field, a confirmed petroleum resource. Moreover, more natural resources might be discovered.

\section{The authorities felt that the composition of public spending reflects adequately} the country's needs. Spending was increased rapidly in recent years to address the people's immediate social needs, laying the foundation of peace and security. Nonetheless, the authorities thought that the mission's recommendations of curbing wage growth, phasing out rice and electricity subsidies, and better targeting transfers to the most needy are not supported by the importance of maintaining overall stability and would have ramifications in a post-conflict and fragile nation. They considered that now is the time to shift the focus to public capital.

\section{EXTERNAL STABILITY AND COMPETITIVENESS}

27. Official dollarization has provided a strong nominal anchor. Dollarization has served the country well and there is currently no obvious alternative. Running an independent monetary policy would be extremely difficult given the absence of a well functioning financial market and limited administrative capacity.

\section{Fiscal policy holds key to competitiveness and external sustainability. Standard} analysis of exchange rate misalignment is currently neither feasible nor very meaningful for Timor-Leste. The lack of data on its nascent economy leaves little scope for econometric techniques. The external current account balance is driven largely by oil and gas revenue, with a small non-oil export sector, contributing only about 3 percent to BoP income. Against this background, it is hard to ascertain a current account norm. The sustainability of the current account in the medium-term is primarily dependent on fiscal policy (Box 1). 
29. Expediting business-enabling reforms is critical to enhance competitiveness and promote private sector-led growth. Commendable progress includes the establishment of a one-stop-shop for investors and the passage of the Transitional Land Law by the Cabinet (though Parliamentary approval is pending). According to the World Bank's Doing Business Indicator, improving land access, easing business start-up, enforcing contract, and enhancing access to finance are key interrelated areas to focus on. Staff welcomed the authorities close cooperation with the World Bank in designing and implementing an action plan guided by the recommendations of the Timor-Leste Diagnostic Trade Integration Study.

\section{Authorities' Views}

30. The authorities agreed that dollarization has served the country well and indicated that there are currently no plans to change the exchange rate regime. However, they raised questions about the impact of dollarization on the competitiveness of Timor-Leste relative to its neighboring countries.

\section{Financial Sector Policy}

\section{The financial sector remains at an embryonic stage of development, with} financial services reaching only a small portion of the population. Three foreign bank branches dominate the financial sector, in addition to a small state-owned microfinance institution and a newly established insurance company. Despite bank deposit growth (30 percent in 2009), private sector credit has remained stagnant since 2005, with surplus funds held mainly abroad, and the loan-to-deposit ratio is about 20 percent. The nonperforming loan to asset ratio has remained at 18 percent since 2008 , but provisioning remains sufficient.
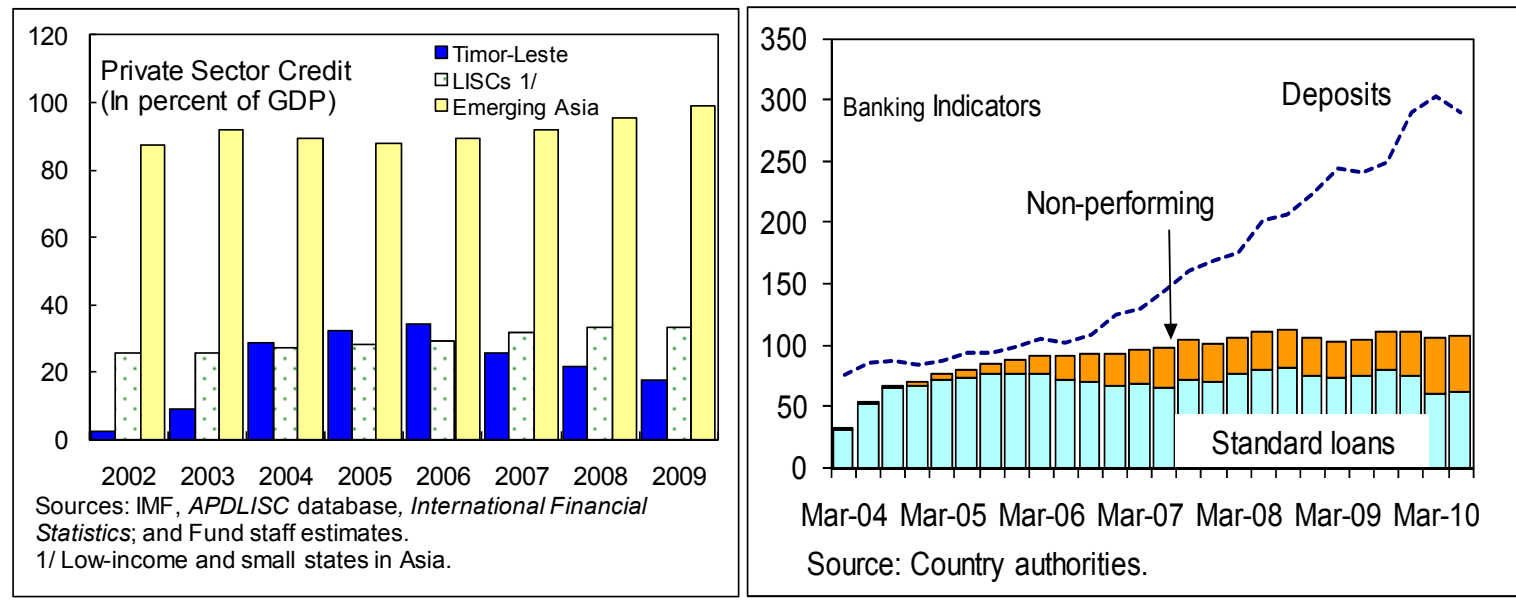

32. Staff suggested speeding up passage of key legislations and regulations to stimulate financial sector development. Unclear property rights, in particular regarding land ownership, and weak contract enforcement prevent efficient collateralization of loans. 
The central bank law to safeguard Banking and Payments Authority's autonomy and the antimoney laundering law were submitted for discussion by relevant Parliament committees.

33. The authorities have continued to strengthen financial supervision. Close coordination with foreign banks' home supervisors is also warranted. Staff encouraged continued efforts of data collection and analysis, and on-site and off-site supervision.

\section{Authorities' Views}

\section{The authorities indicated that commercial banks' high lending rates of 15-} 20 percent are disincentives for credit growth. They expressed interest in receiving technical assistance to promote financial sector development.

\section{Statistics}

35. The availability, quality and timeliness of data continue to pose challenges to policy formulation and surveillance. With extensive technical assistance (TA) from the Fund and other development partners, Timor-Leste has made welcome progress in improving statistics. For the first time, the authorities provided historical non-oil GDP data that entered into staff's analysis. However, important data remain unavailable, and the quality of data, notably national accounts, continues to have significant shortcomings that hamper policy analysis and surveillance. The mission suggested that adequate resources be provided to compile key economic statistics. Participation in the General Data Dissemination System (GDDS) would be beneficial to Timor-Leste.

\section{STAFF APPRAisal}

36. After three years of double-digit expansion, economic growth is likely to slow to about 6 percent in 2010, mainly because adverse weather has slashed agricultural production. As from next year, growth is expected to gradually gather speed and peak at around $93 / 4$ percent in 2013-14, mainly driven by scaling up public investment, but also supported by dynamism in the non-oil private sector, including a recovery in agriculture. In the long term, growth is expected converge to about 6 percent given by supply-side fundamentals. Price pressures have been rising in 2010 and are particularly notable in some segments of the economy, such as construction, and in the skilled labor market. Inflation is expected to increase to 6 percent, somewhat above the average rate in neighboring countries.

37. Timor-Leste should use its oil wealth wisely to build a strong, sustainable nonoil economy that would permanently reduce poverty and raise living standards. The governments' resolve to step up development is admirable. After years of conflict, there is a need to reconstruct basic infrastructure. If well selected and managed, investment in infrastructure could have sufficiently high returns to justify withdrawals from the PF in excess of sustainable income. However, the planned rapid expansion of public investment in infrastructure risks overstretching the country's current ability to plan, evaluate, and 
implement given its institutional weaknesses and administrative capacity constraints. There are also signs of rising inflation as the economy is reaching its absorptive capacity limit, due to the shortage of skilled labor and the small size of the private sector.

38. Therefore, a more-gradual-than-planned pace of spending increases is advisable. Recent years have seen rapid spending growth to address pressing social needs and to lay the foundation for peace in the aftermath of the 2006/7 civil unrest. By 2009, public spending per head has reached a level that is above many comparable countries. The revised higher ESI has created room for additional investment in a limited number of priority projects while gaining experience with the management of capital spending. Spending at a measured pace would stand a better chance of realizing high quality investment, crowding in private investment, and thereby achieve balanced and sustainable growth.

\section{At the same time capacity constraints should be addressed and business-}

enabling reforms implemented. The government's plan to address budget under-execution and to build administrative capacities (to "invest in investing") is encouraging. A new agency, reporting directly to the Prime Minister, will be in charge of major project evaluation, approval, and quality control. It will be important that the agency recruits well-qualified staff. A more efficient administration and business friendlier regulations would make life easier for businesses. The introduction of a one-stop-shop for business is a welcome step in this direction. Reforms to enhance property rights, such as the pending land law should be accelerated. Efficient collateralization would enable the financial sector to extend credits to local businesses.

40. Fiscal policy should be mindful of competitiveness and external stability. Official dollarization has helped keep inflation under control and should be maintained. But dollarization needs to be supported by accelerating productivity-enhancing structural reforms and raising labor skills to improve competitiveness in non-oil industry and services. A slower pace of public spending growth would help to contain inflationary pressures.

41. Staff recommends that the next Article IV consultation be held on the standard 12-month cycle. 


\section{BoX 1. EXCHANGE RATE, COMPETITIVENESS, AND EXTERNAL SUSTAINABILITY ASSESSMENT}

The real effective exchange rate is a poor indicator of Timor-Leste's competitiveness. Lack of data prevents the estimation of equilibrium exchange rates. Moreover, petroleum and coffee, the country's main sources of foreign income, are denominated in U.S. dollars, and the CPI is poorly measured. Competitiveness indicators based on unit costs are unfortunately not available. However, wages, driven by the public sector, have been rising fast, while productivity has been lagging behind and terms-of-trade have improved significantly.

\section{Timor-Leste's business climate has little} improved. The 2010 Doing Business ranking improved due to the 2008 cuts in business and income taxes. However, in terms of overall ranking Timor-Leste is still among the lowest performers out of the 181 countries which participated in the survey. Timor-Leste fell back one rank on the 2010-11 Global Competitiveness ranking, and is at the bottom of the AsiaPacific region. According to the GCI, the most problematic factors for doing business in Timor-Leste are access to financing, poor skills and work ethic, inefficient government bureaucracy, inadequate infrastructure, and corruption. ${ }^{1}$

\section{Current spending plans risk undermining} external stability. From a macroeconomic balance perspective the current account mirrors petroleumfinanced public spending. In 2011, the current account surplus is expected to fall below its sustainable level, computed as the difference between petroleum income and the ESI. In the medium run, the current account surplus is projected to remain below its sustainable level by about 30 percent of GDP.

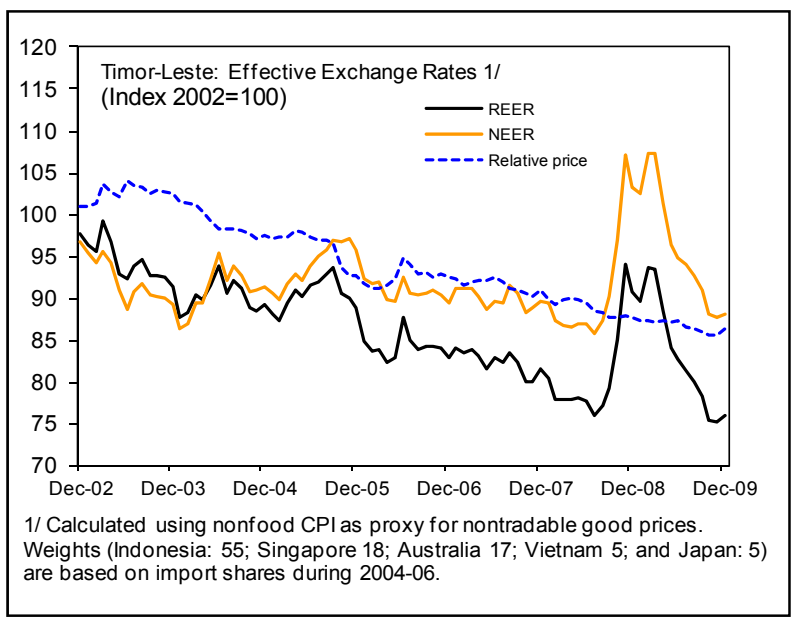

\begin{tabular}{|c|c|c|c|c|c|}
\hline \multicolumn{6}{|c|}{ Assessment of Business Climate, $2009-2010$} \\
\hline \multicolumn{3}{|c|}{ Doing Business } & \multicolumn{3}{|c|}{ Global Competitiveness Index } \\
\hline Countries & $\begin{array}{r}\text { Rank } \\
\text { among } \\
181 \\
(2010)\end{array}$ & $\begin{array}{r}\text { Rank } \\
\text { among } \\
181 \\
(2009)\end{array}$ & Countries & $\begin{array}{r}\text { Rank } \\
\text { among } \\
133 \\
(2010)\end{array}$ & $\begin{array}{r}\text { Rank } \\
\text { among } \\
133 \\
(2009)\end{array}$ \\
\hline Vietnam & 93 & 92 & Thailand & 38 & 36 \\
\hline Papua New Guinea & 102 & 95 & Indonesia & 44 & 54 \\
\hline Sri Lanka & 105 & 102 & Vietnam & 59 & 75 \\
\hline Bangladesh & 119 & 110 & Sri Lanka & 62 & 79 \\
\hline Nepal & 123 & 121 & Philippines & 83 & 87 \\
\hline Indonesia & 122 & 129 & Bangladesh & 103 & 106 \\
\hline Cambodia & 145 & 135 & Cambodia & 105 & 110 \\
\hline Philippines & 144 & 140 & Nepal & 124 & 125 \\
\hline Timor-Leste & 164 & 170 & Timor-Leste & 127 & 126 \\
\hline
\end{tabular}

Sources: World Bank, World Development Indicators; and World Economic Forum.

${ }^{1}$ The Global Competitiveness Report 2010-2011, World Economic Forum. 
Figure 1. Timor-Leste: Regional and Global Comparisons 1/

Timor Leste's living standards remain low in the developing world.
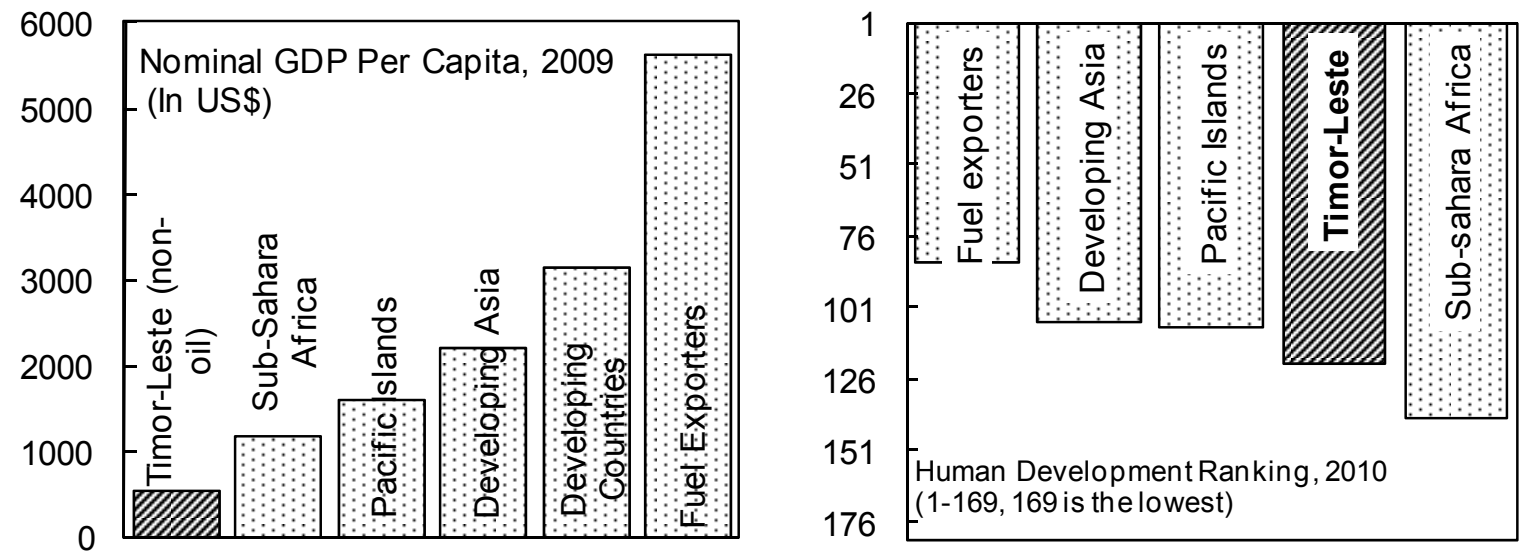

However, if the good macroeconomic policies so far are maintained, with low inflation and no public or external debt,...
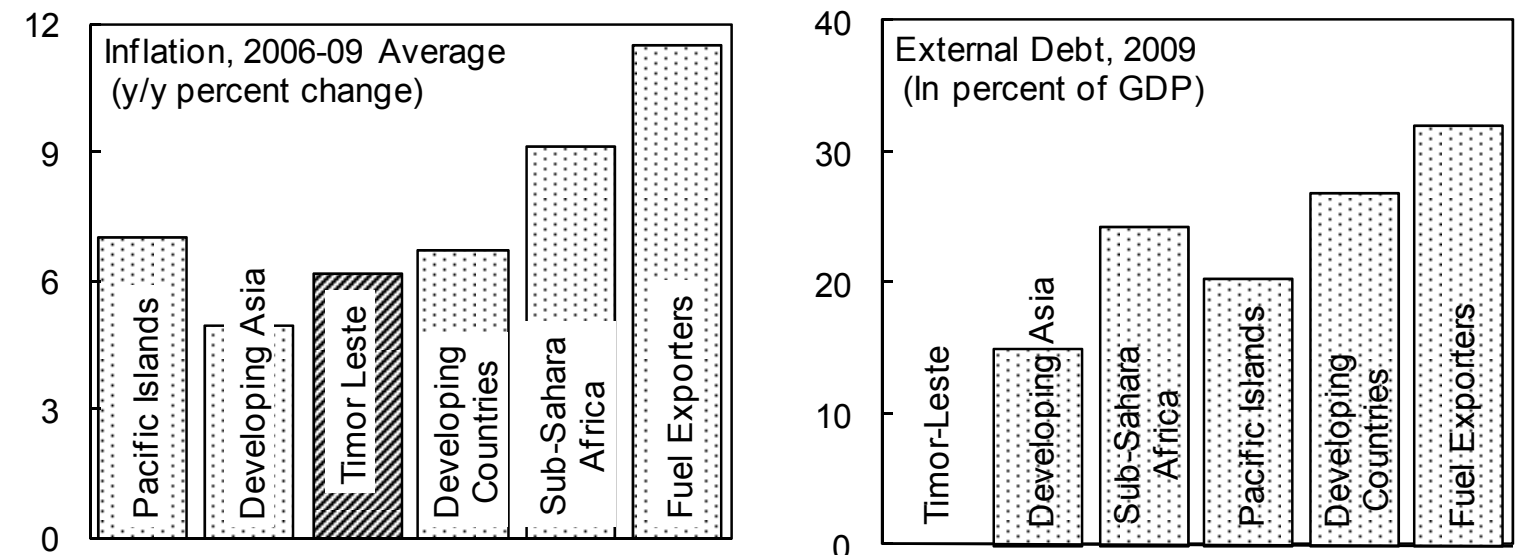

....and the considerable oil wealth is effectively and sustainably harnessed, there is significant scope for promoting non-oil growth.2/
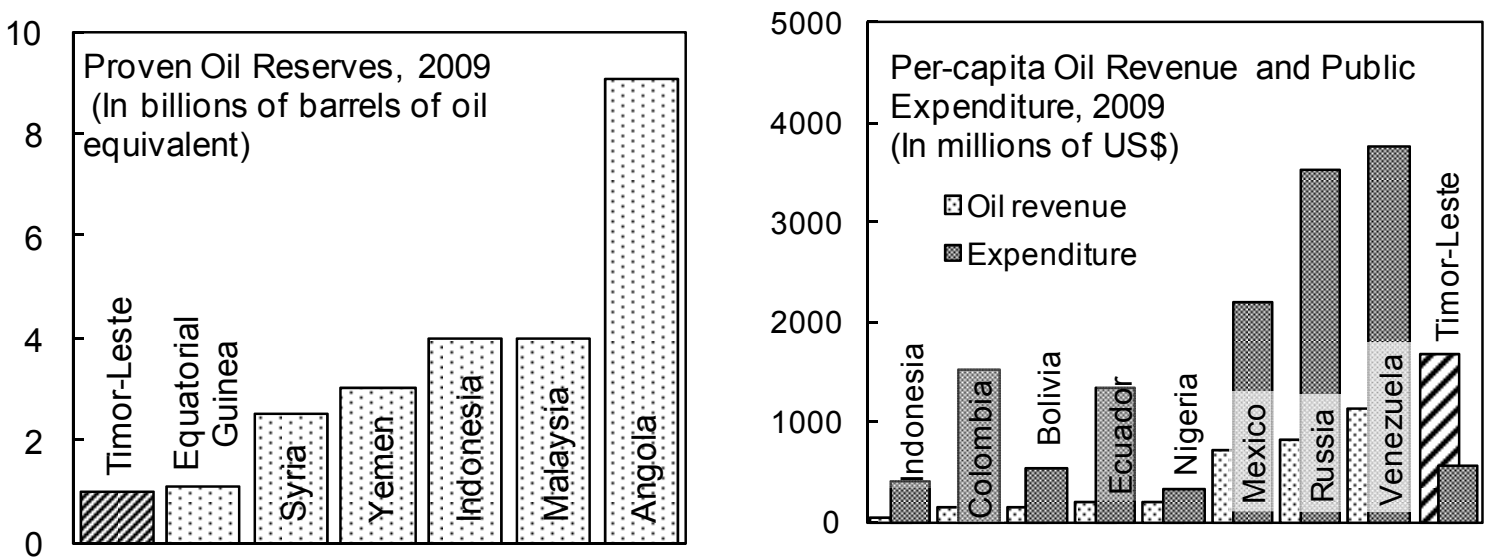

Sources: Authorities; UN, Human Development Indicators, 2009/10; IMF, World Economic Outlook and FAD-DEME databases; PennWell Corporation, Oil \& Gas Journal,December, 2009; and Fund staff estimates.

1/For Timor-Leste, calculations in non-oil GDP.

2/Bayu Undan field only. 
Figure 2. Timor Leste: Recent Macroeconomic Developments

Rapidly rising public spending and an agricultural rebound have fueled high non-oil growth in the last three years. Inflation rose in 2008 along with food prices, but has abated somewhat.
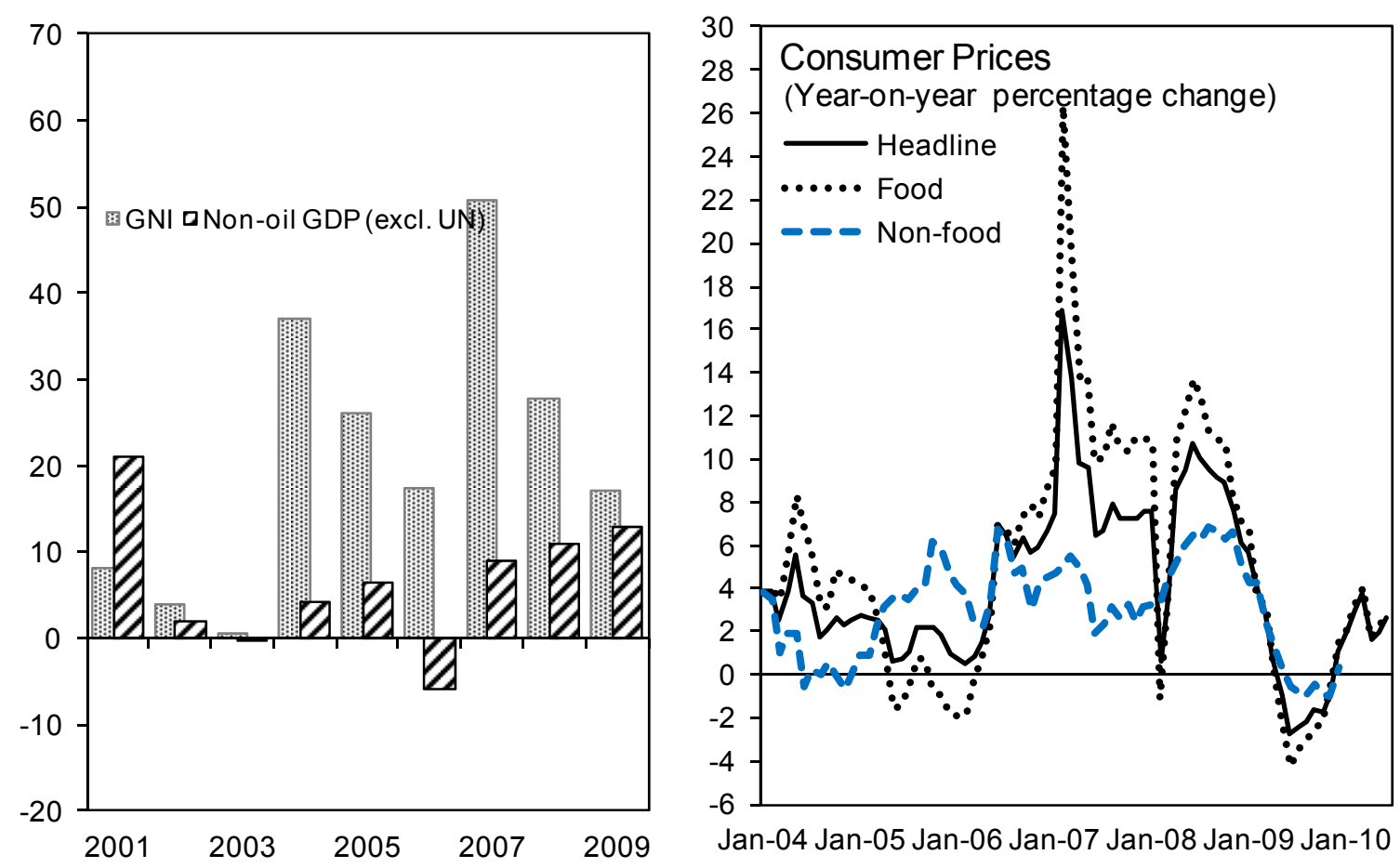

Oil/gas revenue has dominated national income and the current account surplus. Non-oil exports remain very small.
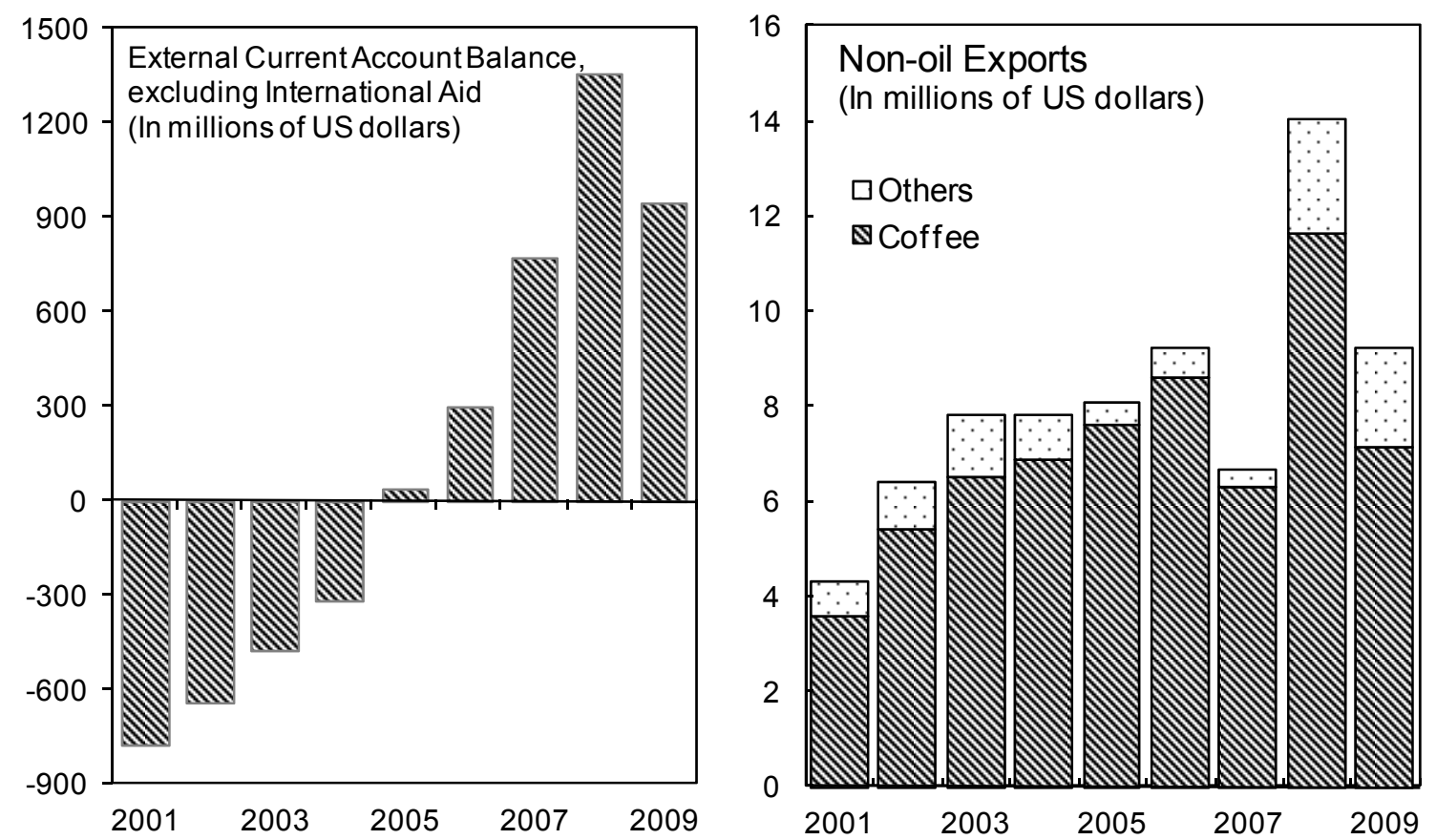

Sources: Timor-Leste authorities; and Fund staff estimates and projections. 
Figure 3: Timor Leste: Medium-term Baseline Scenario
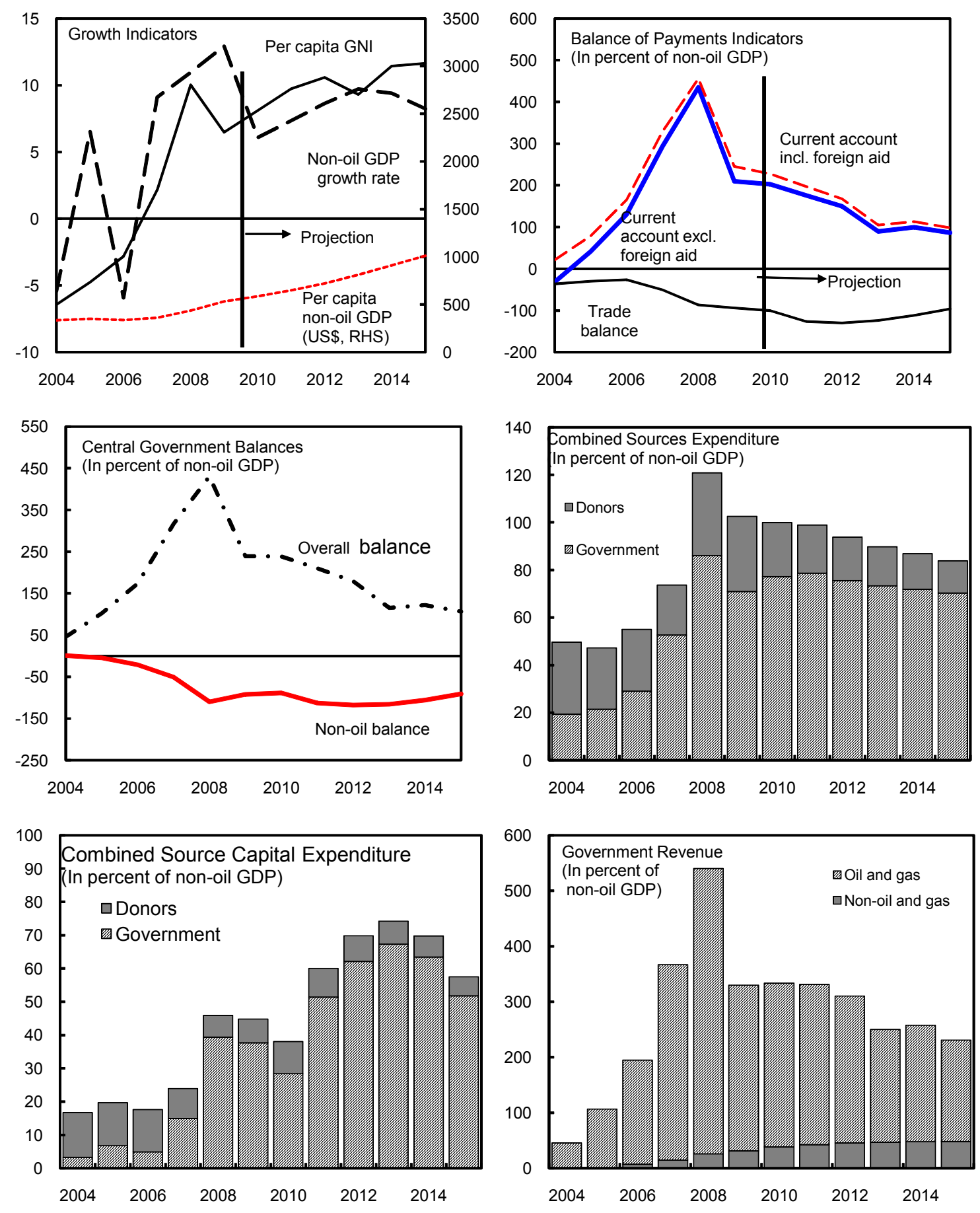

Sources: Country authorities and Fund staff estimates. 
Table 1. Timor-Leste: Selected Social Indicators 1/

\begin{tabular}{|c|c|c|c|c|c|c|}
\hline & $\begin{array}{l}\text { Timor- } \\
\text { Leste }\end{array}$ & & $\begin{array}{c}\text { East Asia } \\
\text { and Pacific } \\
\text { Island } \\
\text { countries }\end{array}$ & & $\begin{array}{c}\text { Low } \\
\text { income } \\
\text { countries }\end{array}$ & \\
\hline Gross national income per capita (US\$) 2/ & 2306 & & 2,644 & & 523 & \\
\hline GDP per capita (PPP, current US\$) & 534 & & 5,446 & & 1,352 & \\
\hline Area (in thousands of square kilometers) & 15 & & 16 & & 28 & \\
\hline \multicolumn{7}{|l|}{ Demography } \\
\hline Total population (in millions) & 1.1 & & 1,930 & & 976 & \\
\hline Population growth (in percent) $3 /$ & 2.4 & & 0.8 & & 2.1 & \\
\hline \multicolumn{7}{|l|}{ Life expectancy and mortality $4 /$} \\
\hline Life expectancy at birth (years) & 61 & & 72.2 & & 59.0 & \\
\hline Male & 60 & & 70.4 & & 57.7 & \\
\hline Female & 62 & & 74.0 & & 60.3 & \\
\hline Infant mortality (per thousand live births) & 67 & & 23.1 & & 75.9 & \\
\hline Under 5 mortality rate (per thousand live births) & 93 & & 28.5 & & 117.9 & \\
\hline \multicolumn{7}{|l|}{ Education (in percent) } \\
\hline Literacy rate $5 /$ & 47 & & 93.1 & & 83.0 & \\
\hline Male & 56 & & 96.0 & & 75.8 & \\
\hline Female & 39 & & 90.1 & & 63.0 & \\
\hline Gross primary school enrollment rate & 107 & & 112.2 & & 100.9 & \\
\hline \multicolumn{7}{|c|}{ Health } \\
\hline \multicolumn{7}{|c|}{ Immunization rate (in percent of children less than 5 years old) } \\
\hline Measles & 57 & & 91.4 & $4 /$ & 78.0 & 4/ \\
\hline DPT & 68 & & 92.3 & $4 /$ & 79.9 & $4 /$ \\
\hline \multicolumn{7}{|l|}{ Other indicators (in percent) } \\
\hline The population below the poverty line & 41 & $6 /$ & $\ldots$ & & $\ldots$ & \\
\hline Households with access to electricity & 36 & & - & & $\ldots$ & \\
\hline Population with access to improved water source & 62 & & 87.0 & $4 /$ & 86.0 & 4/ \\
\hline
\end{tabular}

Sources: World Bank, World Development Indicators; and Country authorities.

1/ Data are for 2009, unless otherwise indicated.

2/ For comparators, GNI is calculated using Atlas method.

3/ Average growth over 2000-09.

4/ Figures are for 2008.

5/ For Timor-Leste, ability to read and write a letter without difficulty among population above age 18.

6/ Figure is for 2009. 
Table 2. Timor-Leste: Selected Social and Economic Indicators

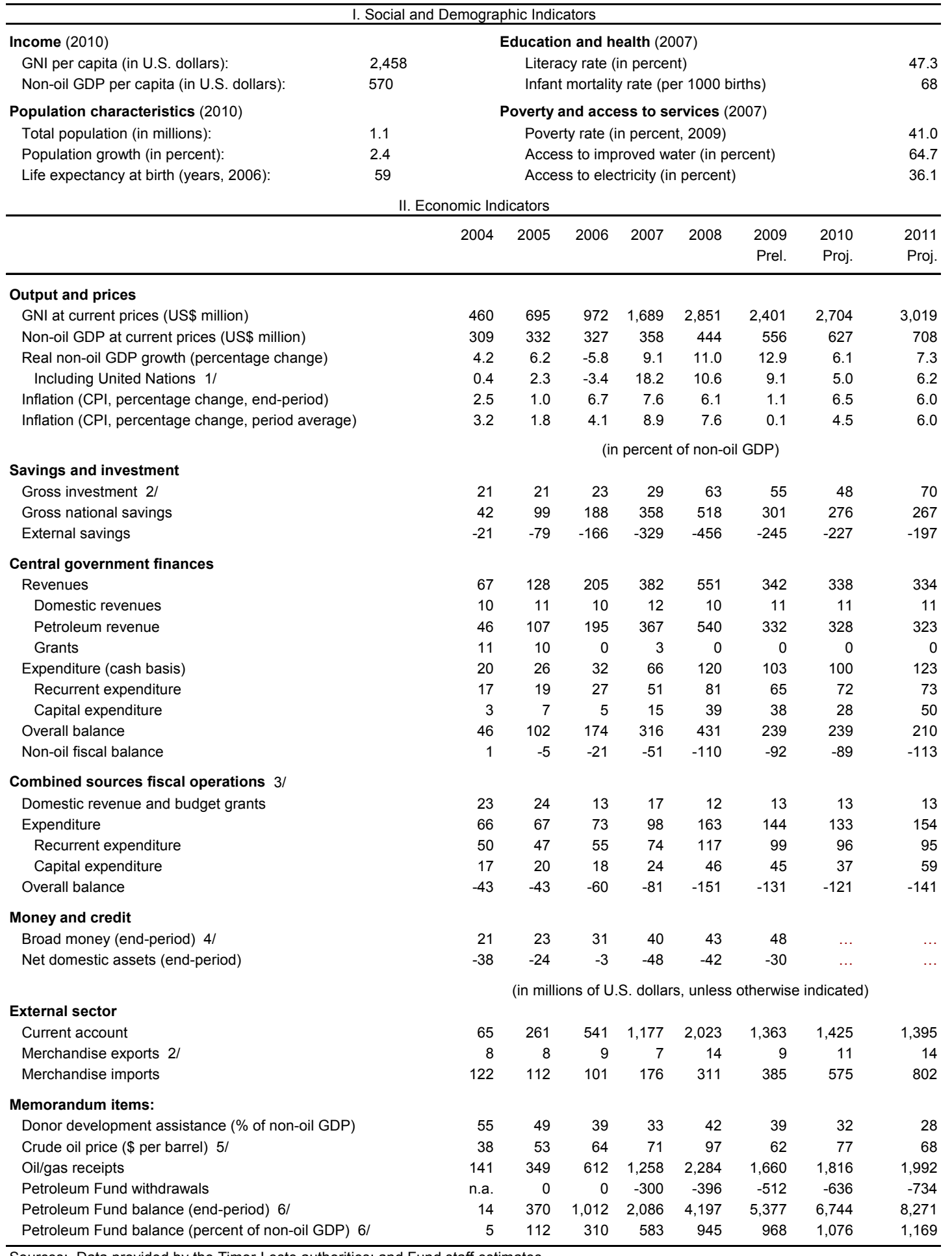

Sources: Data provided by the Timor-Leste authorities; and Fund staff estimates.

$1 /$ Includes locally paid compensation of UN peacekeeping mission staff.

2/ Excludes oil/gas sector.

3/ Includes autonomous agencies and quasi-fiscal expenditure by donors outside the central government budget. The revenue decline in 2005 reflects the creation of the Petroleum Fund to which all oil revenue now accrues. Income from the fund and donor assistance finances the deficit.

4/ Excludes currency holdings by the public, for which no data are available.

5/ Simple average of EIA's low case and reference prices.

6/ Figure for 2004 refers to the Timor-Sea account, which preceded the August 2005 establishment of the Petroleum Fund. 
Table 3. Timor-Leste: Central Government Budget FY2005/06-2011 1/

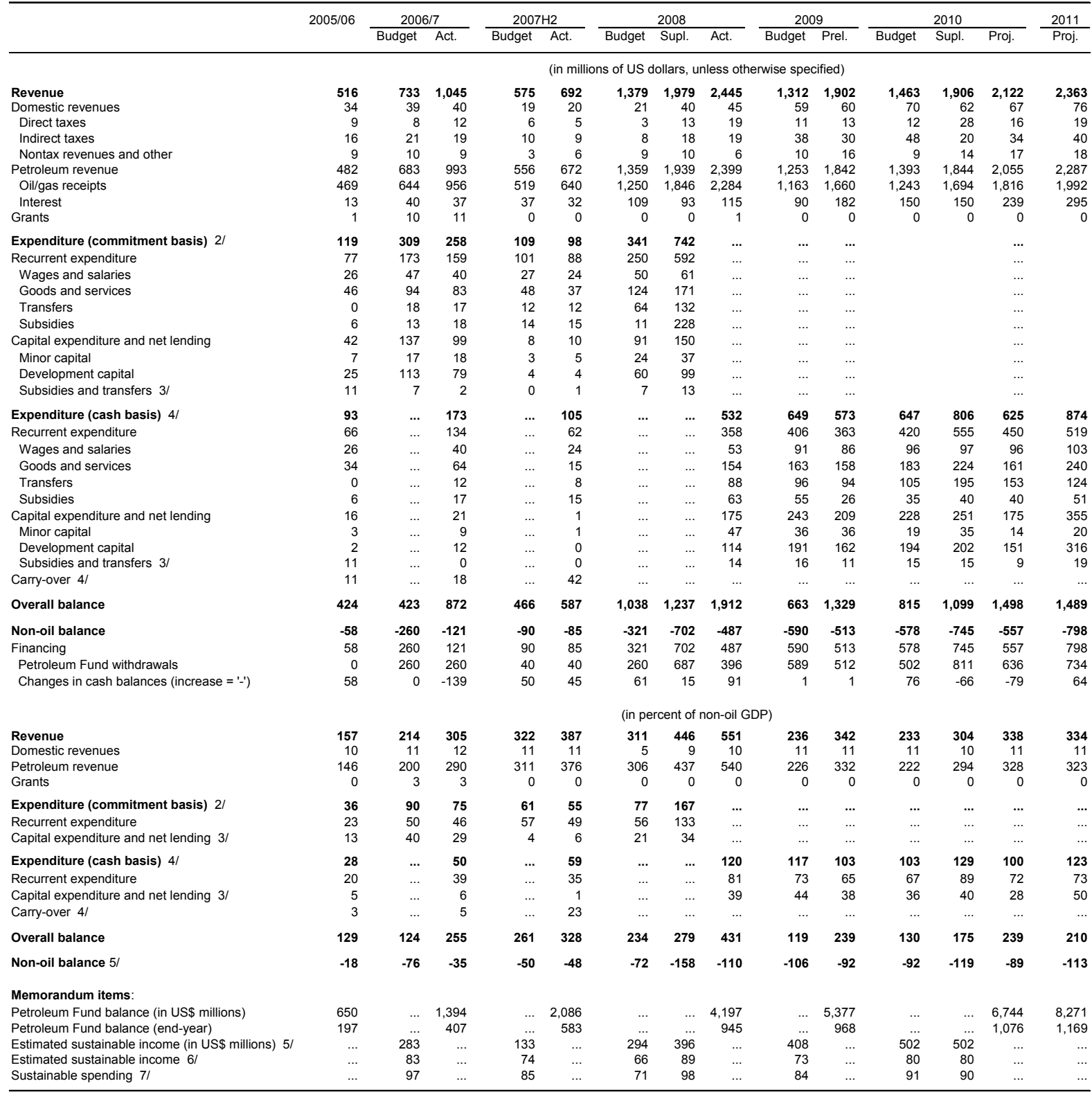

Sources: Timor-Leste authorities; and Fund staff estimates.

1/ Fiscal year: July-June until June 2007; calendar year starting 2008. Central Government includes subsidies to Autonomous Agencies.

Revenue from government rice sales (estimated at $\$ 17$ million in 2008, \$20 million in 2009, and \$14 million in 2010 ) is excluded and only the net subsidy included in recurrent expenditure.

2/ The budget moved toward a cash basis in 2008 and the authorities stopped reporting commitments. Of the $\$ 528$ million reported as spent in 2008 ,

an estimated $\$ 80$ million was actually paid out in 2009 , with spending under the 2008 budget allowed to continue in early 2009 as a transitional

measure before fully applying cash-based principles.

$3 /$ Includes allocations for BPA capitalization (\$10.5 million in 2006/07)

4/ Cash expenditure includes carry-over expenditure from previous years (the reporting of which was not disaggregated prior to 2008).

5/ Defined as overall balance minus petroleum revenue.

6 / Permanent income from oil and gas, estimated according to the Petroleum Fund law for each budget.

7/ Adds non-petroleum revenue to estimated sustainable income from petroleum. 
Table 4. Timor Leste: Combined Sources Budget 2004 - 2011

\begin{tabular}{|c|c|c|c|c|c|c|c|c|}
\hline & 2004 & 2005 & 2006 & 2007 & 2008 & $\begin{array}{l}2009 \\
\text { Prel. }\end{array}$ & $\begin{array}{l}2010 \\
\text { Proj. }\end{array}$ & $\begin{array}{l}2011 \\
\text { Proj. }\end{array}$ \\
\hline & \multicolumn{8}{|c|}{ (in millions of U.S. dollars) } \\
\hline Revenue & 72 & 79 & 42 & 60 & 53 & 70 & 79 & 90 \\
\hline Domestic revenue $1 /$ & 30 & 37 & 34 & 42 & 45 & 60 & 67 & 76 \\
\hline Direct taxes & 7 & 11 & 10 & 13 & 19 & 13 & 16 & 19 \\
\hline Indirect taxes & 19 & 17 & 17 & 19 & 19 & 30 & 34 & 40 \\
\hline Nontax revenues and other & 5 & 9 & 7 & 11 & 6 & 16 & 17 & 18 \\
\hline Autonomous agencies own revenue & 7 & 8 & 7 & 7 & 8 & 10 & 12 & 14 \\
\hline Grant financing (budget support) & 35 & 33 & 1 & 11 & 1 & 0 & 0 & 0 \\
\hline Expenditure (cash basis) & 205 & 222 & 238 & 349 & 724 & 799 & 837 & 1,088 \\
\hline Recurrent expenditure & 154 & 157 & 180 & 263 & 520 & 550 & 602 & 673 \\
\hline Central government and autonomous agencies & 60 & 71 & 95 & 188 & 365 & 374 & 462 & 533 \\
\hline Donor projects $2 /$ & 94 & 86 & 85 & 75 & 155 & 176 & 141 & 141 \\
\hline Capital expenditure & 52 & 66 & 58 & 86 & 204 & 249 & 234 & 415 \\
\hline Central government and autonomous agencies & 10 & 22 & 16 & 53 & 175 & 209 & 175 & 355 \\
\hline Donor projects $2 /$ & 41 & 43 & 42 & 32 & 29 & 40 & 59 & 59 \\
\hline Overall balance & -133 & -144 & -196 & -289 & -671 & -728 & -757 & -998 \\
\hline Financing & 133 & 144 & 196 & 289 & 671 & 728 & 757 & 998 \\
\hline Oil fund financing of central govt. non-oil fiscal deficit & 137 & -4 & 0 & 300 & 396 & 512 & 636 & 734 \\
\hline Project financing by donors $3 /$ & 135 & 129 & 126 & 107 & 184 & 216 & 200 & 200 \\
\hline \multirow[t]{2}{*}{ Change in cash balances (increase -) } & -122 & 21 & 62 & -126 & 4 & -8 & -79 & 64 \\
\hline & \multicolumn{8}{|c|}{ (in percent of non-oil GDP) } \\
\hline Revenue & 23 & 24 & 13 & 17 & 12 & 13 & 13 & 13 \\
\hline Domestic revenue 1/ & 10 & 11 & 10 & 12 & 10 & 11 & 11 & 11 \\
\hline Autonomous agencies own revenue & 2 & 2 & 2 & 2 & 2 & 2 & 2 & 2 \\
\hline Grant financing (budget support) & 11 & 10 & 0 & 3 & 0 & 0 & 0 & 0 \\
\hline Expenditure (cash basis) 2/ & 66 & 67 & 73 & 98 & 163 & 144 & 133 & 154 \\
\hline Recurrent expenditure & 50 & 47 & 55 & 74 & 117 & 99 & 96 & 95 \\
\hline Capital expenditure & 17 & 20 & 18 & 24 & 46 & 45 & 37 & 59 \\
\hline Overall Balance & -43 & -43 & -60 & -81 & -151 & -131 & -121 & -141 \\
\hline Financing & 43 & 43 & 60 & 81 & 151 & 131 & 121 & 141 \\
\hline Oil fund financing of central govt. non-oil fiscal deficit & 44 & -1 & 0 & 84 & 89 & 92 & 101 & 104 \\
\hline Project financing by donors & 44 & 39 & 39 & 30 & 41 & 39 & 32 & 28 \\
\hline Change in cash balances (increase -) & -39 & 6 & 19 & -35 & 1 & -1 & -13 & 9 \\
\hline
\end{tabular}

Sources: Timor-Leste authorities; and Fund staff estimates.

1/ Oil/gas revenue is not included as a budget revenue item and flows directly to the petroleum fund. The amount necessary to cover the non-oil fiscal deficit is withdrawn from the fund and transferred to the budget and recorded as a financing item. Rice sales are excluded from domestic revenue in this table.

2/ Includes sector projects which the government has identified as necessary to achieve development objectives, but may not be wholly

funded by the government.

3/ Donor grants excluding budget support. 
Table 5. Timor-Leste: Balance of Payments 2004-2011

\begin{tabular}{|c|c|c|c|c|c|c|c|c|}
\hline & 2004 & 2005 & 2006 & 2007 & 2008 & $\begin{array}{l}2009 \\
\text { Prel. }\end{array}$ & $\begin{array}{l}2010 \\
\text { Proj. }\end{array}$ & $\begin{array}{r}2011 \\
\text { Proj. }\end{array}$ \\
\hline & \multicolumn{8}{|c|}{ (In millions of U.S. dollars) } \\
\hline Current account & 65 & 261 & 541 & 1,177 & 2,023 & 1,363 & 1,425 & 1,395 \\
\hline Trade balance & -115 & -104 & -91 & -169 & -297 & -376 & -564 & -788 \\
\hline Exports of goods $1 /$ & 8 & 8 & 9 & 7 & 14 & 9 & 11 & 14 \\
\hline Of which: Coffee & 7 & 8 & 9 & 6 & 12 & 7 & 8 & 11 \\
\hline Imports of goods & 122 & 112 & 101 & 176 & 311 & 385 & 575 & 802 \\
\hline Services (net) & -186 & -130 & -198 & -263 & -444 & -505 & -409 & -444 \\
\hline Exports & 47 & 37 & 34 & 63 & 44 & 47 & 68 & 72 \\
\hline Of which: Travel & 19 & 21 & 20 & 26 & 14 & 18 & 20 & 22 \\
\hline Imports & 233 & 167 & 232 & 325 & 488 & 552 & 477 & 516 \\
\hline Of which: UN and donor related & 201 & 137 & 204 & 286 & 364 & 375 & 351 & 345 \\
\hline Income (net) & 151 & 363 & 645 & 1,331 & 2,407 & 1,846 & 2,077 & 2,311 \\
\hline Of which: Oil/gas receipts and interest & 141 & 354 & 637 & 1,312 & 2,399 & 1,842 & 2,055 & 2,287 \\
\hline Current transfers (net) & 215 & 133 & 185 & 278 & 357 & 399 & 321 & 316 \\
\hline Of which: International assistance & 215 & 133 & 184 & 281 & 355 & 362 & 321 & 316 \\
\hline Capital and financial accounts & 56 & -291 & -610 & $-1,031$ & $-2,043$ & $-1,324$ & $-1,338$ & $-1,458$ \\
\hline Official capital transfers & 41 & 43 & 42 & 32 & 17 & 27 & 59 & 59 \\
\hline Financial account $2 /$ & 14 & -334 & -652 & $-1,063$ & $-2,060$ & $-1,351$ & $-1,397$ & $-1,518$ \\
\hline Of which: Oil/gas savings & -3 & -358 & -637 & $-1,012$ & $-2,003$ & $-1,330$ & $-1,419$ & $-1,553$ \\
\hline Overall balance & 121 & -29 & -70 & 147 & -20 & 39 & 88 & -63 \\
\hline \multirow[t]{2}{*}{ Changes in foreign assets (increase -) } & -121 & 29 & 70 & -147 & 20 & -39 & -88 & -64 \\
\hline & \multicolumn{8}{|c|}{ (In percent of non-oil GDP) } \\
\hline Current account & 21 & 79 & 166 & 329 & 456 & 245 & 227 & 197 \\
\hline Trade balance & -37 & -31 & -28 & -47 & -67 & -68 & -90 & -111 \\
\hline Services (net) & -60 & -39 & -61 & -73 & -100 & -91 & -65 & -63 \\
\hline Income (net) & 49 & 109 & 197 & 372 & 542 & 332 & 331 & 327 \\
\hline Current transfers (net) & 70 & 40 & 57 & 78 & 80 & 72 & 51 & 45 \\
\hline Capital and financial accounts & 18 & -88 & -187 & -288 & -460 & -238 & -213 & -206 \\
\hline \multirow[t]{2}{*}{ Overall balance } & 39 & -9 & -21 & 41 & -4 & 7 & 14 & -9 \\
\hline & \multicolumn{8}{|c|}{ (In millions of U.S. dollars) } \\
\hline Memorandum item: & & & & & & & & \\
\hline Public foreign assets (end-period) & 135 & 524 & 1,096 & 2,316 & 4,407 & 5,627 & 7,081 & 8,545 \\
\hline
\end{tabular}

Sources: Data provided by the Timor-Leste authorities, and Fund staff estimates.

1/ Excludes oil/gas receipts, which are recorded under the income account because of lack of detailed data on the oil/gas sector (including production, exports, service payments, and profit remittances).

2/ Includes errors and omissions. 
Table 6. Timor-Leste: Monetary Developments, 2004-2009

\begin{tabular}{|c|c|c|c|c|c|c|}
\hline & 2004 & 2005 & 2006 & 2007 & 2008 & 2009 \\
\hline & \multicolumn{5}{|c|}{ (in millions of U.S. dollars) } & \\
\hline \multicolumn{7}{|l|}{ Banking System } \\
\hline Net foreign assets & 184 & 159 & 113 & 318 & 393 & 443 \\
\hline Assets 1/ & 227 & 206 & 144 & 356 & 422 & 469 \\
\hline Gross reserves & 182 & 153 & 84 & 230 & 210 & 250 \\
\hline Liabilities & 43 & 47 & 31 & 38 & 29 & 26 \\
\hline Net domestic assets & -116 & -80 & -11 & -172 & -187 & -169 \\
\hline Claims on government (net) & -187 & -151 & -83 & -219 & -230 & -206 \\
\hline Claims on private sector & 88 & 106 & 111 & 101 & 102 & 104 \\
\hline Other ("net other assets") & 0 & 0 & 0 & 0 & 0 & 0 \\
\hline Other items, net (incl capital account) & -18 & -35 & -40 & -54 & -60 & -66 \\
\hline Broad money 2/ & 66 & 78 & 100 & 144 & 193 & 268 \\
\hline Narrow money & 32 & 39 & 54 & 75 & 104 & 157 \\
\hline Currency in circulation & 1 & 2 & 2 & 2 & 2 & 3 \\
\hline Demand deposits & 31 & 38 & 52 & 73 & 102 & 155 \\
\hline Other deposits & 34 & 38 & 46 & 68 & 88 & 111 \\
\hline Non-liquid liabilities & 2 & 1 & 2 & 2 & 13 & 5 \\
\hline \multicolumn{7}{|l|}{ Banking and Payments Authority (central bank) } \\
\hline Net foreign assets $1 /$ & 182 & 153 & 84 & 230 & 210 & 238 \\
\hline Assets & 182 & 153 & 84 & 230 & 210 & 250 \\
\hline Gross reserves & 182 & 153 & 84 & 230 & 210 & 250 \\
\hline Others & 0 & 0 & 0 & 0 & 0 & 0 \\
\hline Liabilities & 0 & 0 & 0 & 0 & 0 & 0 \\
\hline Net domestic assets & -175 & -147 & -74 & -210 & -173 & -179 \\
\hline Central Government (net position) & -169 & -129 & -56 & -190 & -196 & -163 \\
\hline Claims on domestic banks and other claims & -8 & -19 & -19 & -20 & 22 & -17 \\
\hline Other items, net & 1 & 1 & 1 & 1 & 1 & 1 \\
\hline Liabilities & 7 & 6 & 9 & 21 & 37 & 59 \\
\hline Currency in circulation $2 /$ & 1 & 2 & 2 & 2 & 2 & 3 \\
\hline Financial institutions & 5 & 5 & 6 & 18 & 23 & 53 \\
\hline Other deposits and liquid liabilities & 0 & 0 & 0 & 0 & 0 & 0 \\
\hline Non-liquid liabilities & 1 & 0 & 1 & 0 & 12 & 3 \\
\hline \multicolumn{7}{|l|}{ Commercial banks $3 /$} \\
\hline Net foreign assets & 1 & 6 & 29 & 88 & 182 & 205 \\
\hline Assets & 44 & 52 & 61 & 126 & 211 & 219 \\
\hline Liabilities & 43 & 47 & 31 & 38 & 29 & 14 \\
\hline Net domestic assets & 65 & 71 & 70 & 55 & 9 & 63 \\
\hline Deposits with BPA & 5 & 3 & 5 & 16 & -19 & 51 \\
\hline Claims on government (net) & -18 & -23 & -27 & -28 & -34 & -44 \\
\hline Claims on private sector & 88 & 106 & 111 & 100 & 102 & 103 \\
\hline Capital accounts & -14 & -15 & 23 & 19 & 12 & 4 \\
\hline Other items (net) & 4 & 1 & -43 & -52 & -52 & -52 \\
\hline Deposit liabilities & 66 & 77 & 99 & 143 & 191 & 266 \\
\hline Demand deposits & 31 & 38 & 52 & 73 & 102 & 155 \\
\hline Time and Savings Deposits & 34 & 38 & 46 & 68 & 88 & 111 \\
\hline Deposits Excluded from Broad Money & 1 & 1 & 1 & 2 & 1 & 0 \\
\hline \multicolumn{7}{|l|}{ Memorandum items } \\
\hline Petroleum Fund balance (US\$ millions) 1/ & $\ldots$ & 370 & 1,012 & 2,086 & 4,197 & 5,377 \\
\hline Net foreign assets (percentage change) & 70.0 & -13.4 & -28.8 & 181.1 & 23.4 & 12.8 \\
\hline Credit to the private sector (percentage change) & 237.2 & 19.8 & 5.2 & -9.8 & 1.9 & 0.9 \\
\hline Credit ratio to non-oil GDP (percent) & 28.6 & 31.9 & 34.1 & 28.1 & 23.1 & 18.6 \\
\hline Broad money ratio to non-oil GDP (percent) & 21.3 & 23.5 & 30.6 & 40.2 & 43.4 & 48.3 \\
\hline Credit ratio to deposits (percent) & 133.9 & 137.4 & 112.5 & 70.1 & 53.5 & 38.9 \\
\hline
\end{tabular}

Sources: Banking and Payments Authority; and Fund staff estimates.

1/ An oil fund was created in September 2005 and the deposits were moved off-shore and onto the Government balance sheet. $2 /$ Includes only coinage issued by the BPA. No data is available for notes due to dollarization of the financial system.

$3 /$ Includes 3 commercial banks (branches of foreign banks) and a micro-finance institution. 
Table 7. Timor-Leste: Medium-Term Outlook, 2006-2015

\begin{tabular}{|c|c|c|c|c|c|c|c|c|c|c|}
\hline & 2006 & 2007 & 2008 & 2009 & 2010 & 2011 & 2012 & 2013 & 2014 & 2015 \\
\hline & & & & & \multicolumn{6}{|c|}{ Projections } \\
\hline \multicolumn{11}{|l|}{ Output and prices } \\
\hline GNI at current prices (in millions of US dollars) & 972 & 1,689 & 2,851 & 2,401 & 2,704 & 3,019 & 3,225 & 3,096 & 3,520 & 3,637 \\
\hline Of which: Non-oil GDP & 327 & 358 & 444 & 556 & 627 & 708 & 806 & 930 & 1,068 & 1,215 \\
\hline Petroleum revenue & 637 & 1,312 & 2,399 & 1,842 & 2,055 & 2,287 & 2,393 & 2,145 & 2,433 & 2,401 \\
\hline Real non-oil GDP growth (percentage change) & -5.8 & 9.1 & 11.0 & 12.9 & 6.1 & 7.3 & 8.6 & 9.7 & 9.4 & 8.2 \\
\hline Including United Nations $1 /$ & -3.4 & 18.2 & 10.6 & 9.1 & 5.0 & 6.2 & 7.4 & 4.2 & 5.4 & 8.2 \\
\hline Inflation (percentage change, period average) & 4.1 & 8.9 & 7.6 & 0.1 & 4.5 & 6.0 & 6.0 & 6.0 & 6.0 & 6.0 \\
\hline & \multicolumn{10}{|c|}{ (in percent of non-oil GDP) } \\
\hline \multicolumn{11}{|l|}{ Investment-saving balance } \\
\hline Gross investment 2/ & 23 & 29 & 63 & 55 & 48 & 70 & 78 & 80 & 74 & 62 \\
\hline of which: public & 18 & 24 & 46 & 45 & 37 & 59 & 67 & 68 & 62 & 49 \\
\hline Gross national saving & 188 & 358 & 518 & 301 & 276 & 267 & 246 & 185 & 187 & 160 \\
\hline External saving & -166 & -329 & -456 & -245 & -227 & -197 & -168 & -105 & -113 & -99 \\
\hline \multicolumn{11}{|l|}{ Central government finances } \\
\hline Revenues & 205 & 382 & 551 & 342 & 338 & 334 & 308 & 242 & 238 & 208 \\
\hline Domestic revenue & 10 & 12 & 10 & 11 & 11 & 11 & 11 & 11 & 11 & 10 \\
\hline Petroleum revenue & 195 & 367 & 540 & 332 & 328 & 323 & 297 & 231 & 228 & 198 \\
\hline Grants & 0 & 3 & 0 & 0 & 0 & 0 & 0 & 0 & 0 & 0 \\
\hline Expenditure & 32 & 66 & 120 & 103 & 100 & 123 & 128 & 126 & 116 & 101 \\
\hline Recurrent expenditure & 27 & 51 & 81 & 65 & 72 & 73 & 69 & 64 & 60 & 57 \\
\hline Capital expenditure & 5 & 15 & 39 & 38 & 28 & 50 & 59 & 62 & 56 & 44 \\
\hline Overall balance & 174 & 316 & 431 & 239 & 239 & 210 & 179 & 115 & 122 & 107 \\
\hline Non-oil fiscal balance & -21 & -51 & -110 & -92 & -89 & -113 & -117 & -116 & -106 & -91 \\
\hline \multicolumn{11}{|l|}{ Combined sources fiscal operations $3 /$} \\
\hline Revenues & 13 & 17 & 12 & 13 & 13 & 13 & 13 & 13 & 13 & 13 \\
\hline Expenditure & 73 & 98 & 163 & 144 & 133 & 154 & 155 & 150 & 137 & 120 \\
\hline Overall balance & -60 & -81 & -151 & -131 & -121 & -141 & -142 & -137 & -125 & -108 \\
\hline & \multicolumn{10}{|c|}{ (in millions of U.S. dollars, unless otherwise specified) } \\
\hline \multicolumn{11}{|l|}{ External sector } \\
\hline Current account balance & 541 & 1,177 & 2,023 & 1,363 & 1,425 & 1,395 & 1,352 & 975 & 1,205 & 1,198 \\
\hline Trade balance & -91 & -169 & -297 & -376 & -564 & -788 & -915 & $-1,006$ & $-1,040$ & $-1,019$ \\
\hline Merchandise exports 4/ & 9 & 7 & 14 & 9 & 11 & 14 & 17 & 23 & 29 & 35 \\
\hline Merchandise imports & 101 & 176 & 311 & 385 & 575 & 802 & 933 & 1,029 & 1,068 & 1,054 \\
\hline Petroleum revenue & 637 & 1,312 & 2,399 & 1,842 & 2,055 & 2,287 & 2,393 & 2,145 & 2,433 & 2,401 \\
\hline Overall balance & -70 & 147 & -20 & 39 & 88 & -63 & 0 & 0 & 0 & 0 \\
\hline Public foreign assets (end-period) & 1,096 & 2,316 & 4,407 & 5,627 & 7,081 & 8,545 & 10,009 & 11,108 & 12,450 & 13,798 \\
\hline (in months of merchandise imports) & 131 & 158 & 170 & 175 & 148 & 128 & 129 & 130 & 140 & 157 \\
\hline & \multicolumn{10}{|c|}{ (in percent of non-oil GDP, unless otherwise specified) } \\
\hline Current account & 166 & 329 & 456 & 245 & 227 & 197 & 168 & 105 & 113 & 99 \\
\hline Trade balance & -28 & -47 & -67 & -68 & -90 & -111 & -114 & -108 & -97 & -84 \\
\hline Merchandise exports 4/ & 3 & 2 & 3 & 2 & 2 & 2 & 2 & 2 & 3 & 3 \\
\hline Merchandise imports & 31 & 49 & 70 & 69 & 92 & 113 & 116 & 111 & 100 & 87 \\
\hline Overall balance & -21 & 41 & -4 & 7 & 14 & -9 & 0 & 0 & 0 & 0 \\
\hline \multicolumn{11}{|l|}{ Memorandum items: } \\
\hline Crude oil price ( $\$$ per barrel) $5 /$ & 64 & 71 & 97 & 62 & 77 & 68 & 71 & 75 & 79 & 81 \\
\hline Petroleum Fund withdrawals & 0 & -84 & -89 & -92 & -101 & -104 & -115 & -112 & -102 & -87 \\
\hline Petroleum Fund balance (end-period) & 310 & 583 & 945 & 968 & 1,076 & 1,169 & 1,208 & 1,165 & 1,140 & 1,113 \\
\hline
\end{tabular}

Sources: Data provided by the Timor Leste authorities; and Fund staff estimates and projections.

$1 /$ Includes locally paid compensation of UN peacekeeping mission staff.

2/ Excludes oil/gas sector investment.

$3 /$ Includes autonomous agencies and fiscal and quasi-fiscal expenditure programs undertaken by bilateral donors and international financial institutions outside the central government budget.

4/ Excludes oil/gas revenue, which is recorded under the income account.

5/ Simple average of EIA's low case and reference prices. 
Table 8. Timor-Leste: Vulnerability Indicators, 2004-2009

\begin{tabular}{|c|c|c|c|c|c|c|}
\hline & 2004 & 2005 & 2006 & 2007 & 2008 & 2009 \\
\hline \multicolumn{7}{|l|}{ Financial sector risk indicators } \\
\hline Public debt $1 /$ & 0.0 & 0.0 & 0.0 & 0.0 & 0.0 & 0.0 \\
\hline Broad money (percent change, 12-month basis) $2 /$ & & 18.3 & 28.2 & 43.9 & 34.1 & 39.3 \\
\hline Private Sector credit (percent change, 12-month basis) & & 19.8 & 5.2 & -9.8 & 1.9 & 1.1 \\
\hline \multicolumn{7}{|l|}{ External indicators } \\
\hline Goods exports (percent change, 12-month basis in U.S. dollars) & 0 & 3 & 14 & -28 & 111 & -34 \\
\hline Goods imports (percent change, 12-month basis in U.S. dollars) & 6 & -8 & -11 & 75 & 77 & 24 \\
\hline Current account balance & 21 & 79 & 166 & 329 & 456 & 245 \\
\hline Public foreign assets (in millions of U.S. dollars) $3 /$ & 182 & 524 & 1,096 & 2,316 & 4,407 & 5,627 \\
\hline (in months of corresponding imports of goods) & 18 & 56 & 131 & 158 & 170 & 175 \\
\hline Net Foreign assets of commercial banks (in millions of U.S. dollars) & 1 & 6 & 29 & 88 & 182 & 205 \\
\hline Total external debt $1 /$ & 0 & 0 & 0 & 0 & 0 & 0 \\
\hline External debt-service (in percent of exports of goods and services) & 0 & 0 & 0 & 0 & 0 & 0 \\
\hline
\end{tabular}

Sources: Timor-Leste authorities; and Fund staff estimates.

$1 /$ No external or domestic debt has been contracted.

2/ Excludes currency in circulation on which no data are available.

3 / Includes investments of Petroleum Fund assets overseas. 


\begin{tabular}{|c|c|c|c|c|c|}
\hline & 1990 & 1995 & 2000 & 2005 & 2008 \\
\hline \multicolumn{6}{|l|}{ Goal 1: Eradicate extreme poverty and hunger } \\
\hline \multicolumn{6}{|l|}{ Target 1.A: Halve, between 1990 and 2015, the proportion of people whose income is less than $\$ 1$ a day } \\
\hline Poverty gap at $\$ 1.25$ a day (PPP) $(\%)$ & .. & .. & 19 & 9 & 9 \\
\hline Poverty headcount ratio at $\$ 1.25$ a day (PPP) (\% of population) & .. & .. & 53 & 37 & 37 \\
\hline Income share held by lowest $20 \%$ & .. & .. & 7 & 9 & 9 \\
\hline Malnutrition prevalence, weight for age ( $\%$ of children under 5 ) & .. & .. & 41 & .. & .. \\
\hline \multicolumn{6}{|c|}{ Target 1.B: Achieve full and productive employment and decent work for all, including women and young people } \\
\hline Employment to population ratio, $15+$, total $(\%)$ & 64 & 64 & 65 & 65 & 67 \\
\hline Employment to population ratio, ages $15-24$, total $(\%)$ & 51 & 51 & 51 & 55 & 58 \\
\hline \multicolumn{6}{|l|}{ Goal 2: Achieve universal primary education } \\
\hline \multicolumn{6}{|c|}{ Target 2.A: Ensure that, by 2015 , children everywhere, boys and girls alike, will be able to complete a full course of primary schooling } \\
\hline Primary completion rate, total (\% of relevant age group) & .. & .. & .. & 70 & 80 \\
\hline Total enrollment, primary (\% net) & .. & .. & .. & 69 & 77 \\
\hline \multicolumn{6}{|l|}{ Goal 3: Promote gender equality and empower women } \\
\hline \multicolumn{6}{|c|}{ Target 3.A: Eliminate gender disparity in primary and secondary education, preferably by 2005 , and in all levels of education no later than 2015} \\
\hline Proportion of seats held by women in national parliaments $(\%)$ & .. & .. & .. & 25 & 29 \\
\hline Ratio of female to male primary enrollment (\%) & .. & .. & .. & 92 & 94 \\
\hline Ratio of female to male secondary enrollment $(\%)$ & .. & .. & .. & 100 & .. \\
\hline Ratio of female to male tertiary enrollment (\%) & .. & .. & 127 & .. & 71 \\
\hline Share of women employed in the nonagricultural sector (\% of total nonagricultural employment) & .. & .. & 35.0 & .. & .. \\
\hline \multicolumn{6}{|l|}{ Goal 4: Reduce child mortality } \\
\hline \multicolumn{6}{|l|}{ Target 4.A: Reduce by two-thirds, between 1990 and 2015, the under-five mortality rate } \\
\hline Mortality rate, infant (per 1,000 live births) & 138 & 114 & 84 & 61 & 51 \\
\hline Mortality rate, under-5 (per 1,000$)$ & 184 & 150 & 106 & 74 & 60 \\
\hline \multicolumn{6}{|l|}{ Goal 5: Improve maternal health } \\
\hline \multicolumn{6}{|l|}{ Target 5.A: Reduce by three quarters, between 1990 and 2015, the maternal mortality ratio } \\
\hline Maternal mortality ratio (modeled estimate, per 100,000 live births) & 650 & 590 & 520 & 420 & 370 \\
\hline Births attended by skilled health staff ( $\%$ of total) & .. & 26 & 24 & 18 & .. \\
\hline \multicolumn{6}{|l|}{ Target 5.B: Achieve, by 2015, universal access to reproductive health } \\
\hline Contraceptive prevalence (\% of women ages $15-49)$ & .. & .. & 8 & 20 & 20 \\
\hline Adolescent fertility rate (births per 1,000 women ages $15-19$ ) & .. & .. & 89 & 59 & 53 \\
\hline Pregnant women receiving prenatal care (\%) & .. & .. & 43 & 61 & .. \\
\hline \multicolumn{6}{|l|}{ Goal 6: Combat HIVIAIDS, malaria, and other diseases } \\
\hline \multicolumn{6}{|l|}{ Target 6.C: Have halted by 2015 and begun to reverse the incidence of malaria and other major diseases } \\
\hline Tuberculosis case detection rate (all forms) & .. & & 64 & 76 & 60 \\
\hline Incidence of tuberculosis (per 100,000 people) & 500 & 500 & 500 & 500 & 500 \\
\hline $\begin{array}{l}\text { Children with fever receiving antimalarial drugs (\% of children under age } 5 \text { with fever) } \\
\text { Goal 7: Ensure environmental sustainability }\end{array}$ & .. & .. & 47 & .. & .. \\
\hline
\end{tabular}

Target 7.A: Integrate the principles of sustainable development into country policies and programmes and reverse the loss of environmental resources

Target 7.B: Reduce biodiversity loss, achieving, by 2010, a significant reduction in the rate of loss

Forest area (\% of land area)

Marine protected areas ( $\%$ of total surface area)

Terrestrial protected areas (\% of total surface area)

$\begin{array}{lllll}65.0 & 61.2 & 57.4 & 53.7 & 52.2\end{array}$

Improved sanitation facilities (\% of population with access)

Improved water source (\% of population with access)

Goal 8: Develop a global partnership for development

Target 8: Various

Net ODA received per capita (current US\$)

Other

Fertility rate, total (births per woman)

GNI per capita, Atlas method (current US\$)

GNI, Atlas method (current US\$) (billions)

Gross capital formation (\% of GDP)

Life expectancy at birth, total (years)

$\begin{array}{lr}. . & . . \\ . . & . . \\ . . & . .\end{array}$

.. $\quad$.

0

15

$32 \quad 44 \quad 50$

$52 \quad 63 \quad 69$

Figures in italics refer to periods other than those specified.

Source: World Development Indicators database, 2010. 
Appendix Table 1. Authorities' Response to Recent Fund Policy Advice 1/

\begin{tabular}{l} 
Fund Recommendations \\
\hline \hline Monetary and Exchange Rate Policy \\
Official dollarization has helped keep inflation under control, and \\
should be maintained. \\
Fiscal Policy \\
Public spending should return to a sustainable path over the \\
medium term, and be targeted to growth promotion and poverty \\
reduction.
\end{tabular}

Policy Actions

Monetary and Exchange Rate Policy

Official dollarization has helped keep inflation under control, and Continued commitment to build up institutional capacity before should be maintained. any policy change.

Spending growth in 2009 has been moderate and cash expenditures are expected to remain below sustainable levels in 2010. However, the authorities plan to step up capital spending in 2011-15 to promote growth and poverty reduction.

Fiscal prudence is also needed to support competitiveness and external sustainability.

The authorities are aware of competitiveness problems but consider infrastructure investment a priority.

Petroleum Fund remain at the center of petroleum based revenue management.

Current draft amendment to Petroleum Fund law leaves its core principles intact, and increases flexibility in investment and budget process. However, accountability to parliament might be weakened.

A medium-term expenditure framework should be introduced, and capital investment projects should be carefully prioritized and costed.

The Strategic Development Plan 2011-30 lays out the five-year public investment budget plan with sectoral priorities. But details have to be worked out and administrative capacity constraints hamper public investment management.

Foreign borrowing should be subject to Parliamentary approval and proper justification.

The authorities are considering the option of borrowing, and parliament approval has been made a legal requirement.

\section{Financial Sector Policy}

Continued efforts are needed to strengthen financial sector supervision and promote financial development.

Banking supervision enhancement continues. As part of the effort to improve financial sector supervision, the authorities established the Credit Registry Agency.

\section{Structural Reforms}

Reforms to enhance property rights are also needed to promote Land Law progressing but other steps are still pending. financial development.

Source: IMF Staff.

1/ Advice from the 2009 Article IV Consultation.

\section{CInternational Monetary Fund. Not for Redistribution}




\section{Annex 1. Timor-Leste: Strategic Development Plan ${ }^{1}$}

\section{In April 2010, the Timor-Leste authorities published a summary of Strategic} Development Plan (SDP) for 2011-2030, with the full SDP yet to be officially released. Immediately after the publication, the Prime Minister started country-wide consultation of the SDP.

The SDP summary defines challenges faced by the country. It outlines target growth rates for public investment and financing until 2015, provides a "framework of action until 2020" and the government's "visions" of the country's stage of development in 2030. The authorities plan to establish an Economic Planning and Investment Agency (EPIA) in charge of the design, budgeting, and implementation of the SDP.

- Main challenges that the SDP will address include urban-rural and regional imbalances, shortage of skilled human resources, fragile institutions of the state, and post-conflict mentality.

- The SDP aims to put Timor-Leste among the upper-middle-income countries by 2030, supported by strong growth of the non-oil economy at an average rate of 12 percent per year during 2010-20 and 10 percent per year during 2020-30.

- With public investment taking the lead, the framework of action to 2020 entails major public and private investment projects in human capital, infrastructure, and strategic sectors such as agriculture, petroleum (including downstream), and tourism. Improving the business environment is also seen as a fundamental priority.

- The public investment plan to 2015 defines a five-year public investment budget in the order of 30 percent of GNP per year and focuses on eight priority areas: health and nutrition, education and research, roads, power, seaports and airports, agriculture, petroleum, and tourism. Financing options of the public investment will include withdrawals from the PF in excess of the ESI or concessional borrowing. The government intends to keep current expenditure within the ESI.

\footnotetext{
${ }^{1}$ Based on the 2010 "Public Financial Management-Performance Report" and the "Report on Observance of Standards and Codes (ROSC)—Fiscal Transparency Module."
} 


\section{ANNEX 2. The ESTIMATED SUSTAINABLE INCOME}

- The Petroleum Fund (PF) Law defines the estimated sustainable income (ESI) as 3 percent of Timor-Leste's total petroleum wealth, including the current PF balance plus the net present value of future petroleum receipts.

- Rather than a rigid fiscal ceiling, the ESI is a benchmark to guide budget withdrawals from the PF and inform policy discussions. Actual withdrawals can exceed the ESI as long as the government delivers a detailed explanation to parliament why doing so is in the long-run interest of country.

- The ESI is calculated every year based on a set of assumptions on petroleum output and prices. The PF Law requires prudent assumptions to be used, as 70 percent of TimorLeste's oil wealth remains under the sea and there is no diversification across multiple projects.

- Only projects with an approved development plan and firm investment commitment (i.e., the Bayu Undan and Kitan fields) have been included in the ESI calculation. The Greater Sunrise field has been declared a commercial discovery, but does not have an approved development plan, and therefore according to current policy should not be included in ESI. Further, its exclusion appears justified given the material uncertainties surrounding the technical and commercial configuration of the project and the potential for significant delays. However, Sunrise is a confirmed petroleum resource and its exclusion from ESI arguably puts some strain on the credibility of the policy.

- Nevertheless, the Ministry of Finance should begin evaluating its potential effect on petroleum wealth and fiscal financing. Perceived petroleum wealth from Sunrise is already informing wider thinking in government and civil society about sustainable spending levels, and quantification of the potential revenue flows would help to inform and guide that discussion. In particular, this exercise would allow the authorities to manage expectations and encourage a more realistic assessment of the potential addition to petroleum wealth from Sunrise.

- Until 2010, the oil price used for ESI calculation has been the "low" case from the U.S. Energy Information Administration (EIA) Annual Energy Outlook. To address the concern within the government that the low case lies materially below market and other respected forecasters expected prices, the authorities recently explored other alternative methodologies for determining a price forecast more reflective of expectations but still prudent. It came to a conclusion of using the average of the EIA low and reference cases, which balances between being realistic and prudent. For 2011, the average price generates an ESI of \$734 million compared to \$548 million under the EIA low case price. 


\section{AnNeX 3. Timor-Leste: Fiscal ROSC ANd PEFA Assessments}

\section{The 2010 fiscal ROSC and PEFA exercises evaluated the strengths and weaknesses of} Timor-Leste's public financial management (PFM) system. In response to the authorities' request, the Fund carried out both exercises with participation of the WB and the ADB. This diagnostic work will form the basis for the IMF's input in helping the authorities elaborate a comprehensive PFM reform strategy in close cooperation with the World Bank.

\section{The assessments suggest that overall Timor-Leste has made solid progress in} strengthening the PFM system from a low base. Key strengths in fiscal transparency include: a well-structured, basic budget process; a well-managed and supervised framework for the petroleum sector; comprehensive budget documentation and regular fiscal reporting; a modern integrated financial management information system; and an efficient Treasury Single Account. Notable gains have also been made in funding predictability, timeliness and quality of bank reconciliation and financial statements, legislative scrutiny, functionality of the treasury system, and external audit process.

\section{However, substantial weaknesses in the PFM system remain.}

- $\quad$ The budget is not a good predictor of actual spending, mainly reflecting large supplementary budgets.

- $\quad$ Budget Review in the Ministry of Finance has very little assessment capacity, especially on the capital side.

- $\quad$ Budget planning and implementation capacity in line ministries are still very weak. Budget oversight of autonomous government agencies and public enterprises, albeit relatively few in number, is weak and associated fiscal risks are not monitored.

- Independent internal and external audit process is lacking.

- $\quad$ Procurement has been in a state of flux for many years, with less competitive instruments becoming more prevalent.

- $\quad$ The budget is only weakly linked to policy objectives and the implications of capital spending for recurrent budgets are ignored.

- The Ministry of Finance has little capacity to adequately review the rationale, costing, and impact of public investment.

- A solid multi-year perspective on fiscal planning, expenditure policy, and budgeting is missing.

Despite improved transparency in tax payer obligations, compliance and tax audit remain a major issue. 


\section{ANNEX 4. INDEX OF BUDGET INSTITUTIONS}

Staff constructed two inter-related indexes of budget institutions to capture the quality of budget institutions in Timor-Leste. ${ }^{1}$ The first dimension (stage index) is along three budget stages including planning, approval, and implementation. The second dimension (category index) covers five cross-cutting categories at each budgetary stage: top-down procedures; rules and controls; sustainability and credibility; comprehensiveness; and transparency. Based on the recent fiscal ROCS and PEFA results, a score ranging from 0-4 is assigned to each dimension and category according to a common scoring methodology documented in Dabla-Norris et al (2010). The overall stage index and category index are simple averages of sub-indexes.

\section{Results suggest that Timor-Leste's budget institutions are relatively strong on} transparency and comprehensiveness, but score below low-income countries (LICs) on average. The overall stage index (1.6) is lower than the average of LICs (1.9), reflecting weaknesses in budget planning and approval. Important shortcomings include (i) unclear fiscal targets; (ii) a dual budget process where capital and current budgets are developed separately; and (iii) inadequate analysis of fiscal risks. The overall category index (1.7) is also lower than the average of LICs (1.9). Particularly, Timor-Leste scores poorly in fiscal rules and controls, and sustainability and credibility, reflecting the absence of a solid medium-term fiscal perspective, large gaps in internal and external audit, and inadequate monitoring of fiscal risks. A commendable achievement for Timor-Leste is its relatively high fiscal transparency, which is measured to be close to the average of middle-income countries (MICs). Budget documents also provide fairly comprehensive information compared with those of many other LIC peers.

\footnotetext{
${ }^{1}$ The methodology is taken from "Budget Institutions and Fiscal Performance in Low-Income Countries" by E. Dabla-Norris et al (IMF Working Paper 10/80). The lower is the index, the weaker are budget institutions.
} 


\section{Timor-Leste: Stage Index of Budget Institutions}

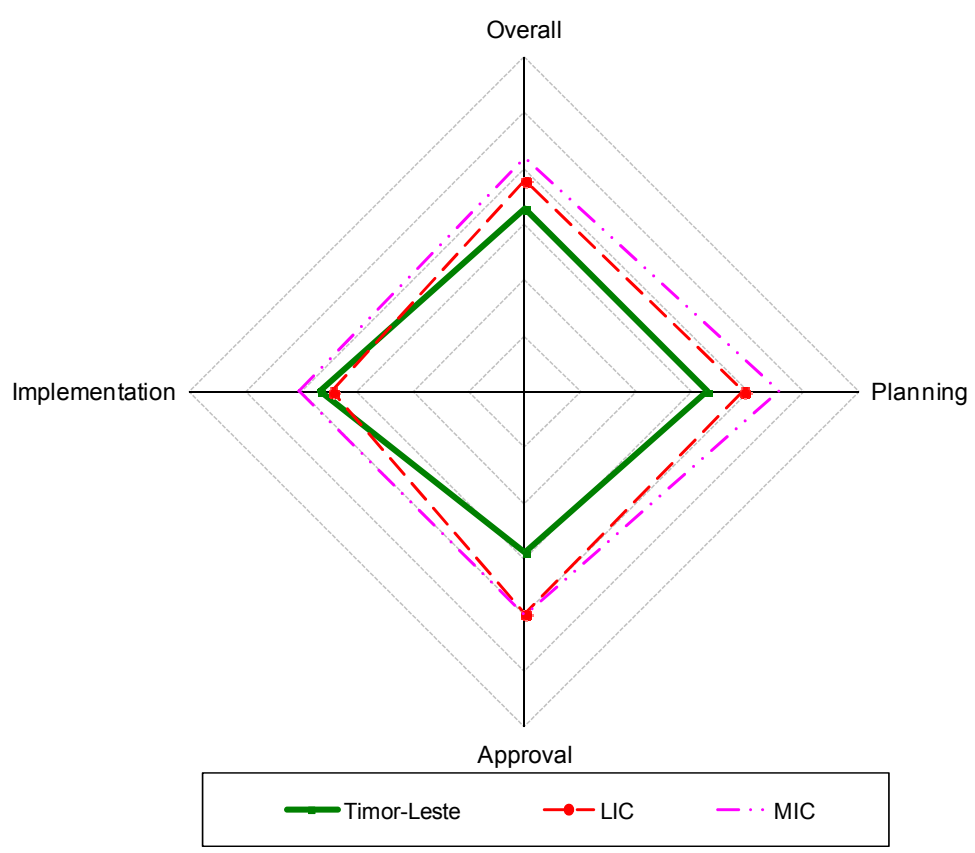

Timor-Leste: Category Index of Budget Institutions

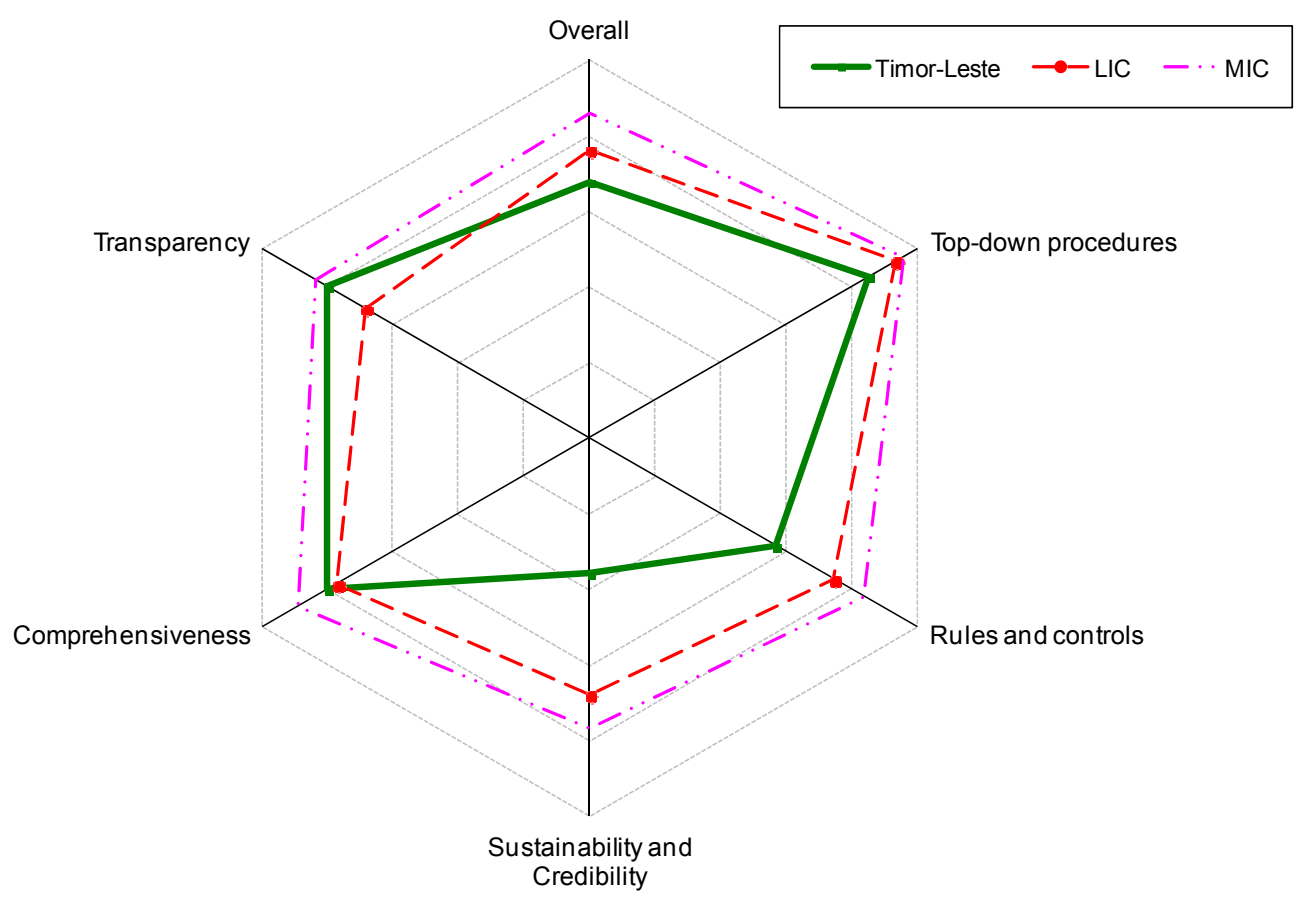

CInternational Monetary Fund. Not for Redistribution 
This page intentionally left blank

CInternational Monetary Fund. Not for Redistribution 


\section{INTERNATIONAL MONETARY FUND \\ DEMOCRATIC REPUBLIC OF TIMOR-LESTE}

\section{Staff Report for the 2010 Article IV Consultation-Informational Annex}

Prepared by the Staff Representatives for the 2010 Consultation with the Democratic Republic of Timor-Leste

(In Consultation with Other Departments)

December 3, 2010

Contents

Page

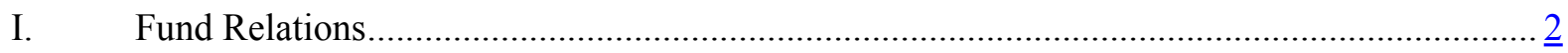

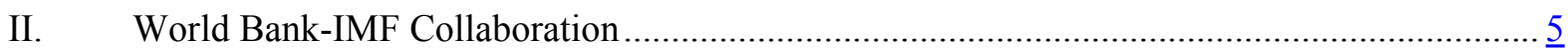

III. Relations with the Asian Development Bank .............................................................. $\underline{8}$

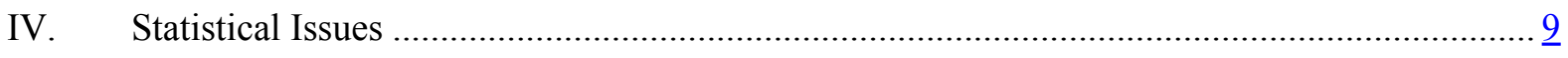




\section{ANNEX I. TIMOR-LESTE: FUND RELATIONS}

(As of November 01, 2010)

I. Membership Status: Joined 7/23/2002; Article VIII

II. General Resources Account:

SDR Million

$\%$ Quota

Quota

100.00

Fund holdings of currency

8.20

100.00

Reserve position in Fund

0.00

0.01

III. SDR Department:

SDR Million

7.73

7.73
\% Allocation

100

100

IV. Outstanding Purchases and Loans: None

V. Latest Financial Arrangements: None

VI. Projected Obligations to Fund: None

VII. Exchange Rate Arrangements

On January 24, 2000, the U.S. dollar was adopted as the official currency of then East Timor by the United Nations Transitional Administration in East Timor (UNTAET). This arrangement has been maintained after Timor-Leste's independence on May 20, 2002. At present, the monetary authority does not undertake foreign exchange transactions; they are handled by commercial banks on the basis of rates quoted in the international markets.

\section{Article IV Consultations}

The 2009 Article IV consultation discussions with the authorities were held during June 4-15, 2009; the consultation was concluded on June 15, 2009. The Public Information Notice may be found at http://www.imf.org/external/np/sec/pn/2009/pn0988.htm.

\section{Technical Assistance}

Since late 1999, a significant amount of technical assistance has been provided by the Fund as part of the international community's efforts to reconstruct the Timor-Leste economy. The Fund's technical assistance has focused on establishing key economic institutions (especially fiscal and monetary authorities) essential for macroeconomic management, as well as on developing 
local capacity to manage them. It also played a key role in the adoption of a new currency arrangement based on the U.S. dollar. Since late 1999, Timor-Leste has been one of the largest recipients of Fund technical assistance.

FAD: A series of multi-topic FAD missions took place early on to establish and develop the Central Fiscal Authority (CFA, February 2000), which developed into the Ministry of Planning and Finance. Assistance was provided to develop budget preparation, establish tax policy and administration, strengthen treasury and expenditure management, and advise on oil sector fiscal regimes. Long-term resident advisors were assigned to the Ministry of Planning and Finance and the Revenue Service of Timor-Leste to cover a wide range of issues, partly under a cost sharing arrangement with the UN. Over August 2004-July 2005, a FAD resident advisor assisted in the design and establishment of the Petroleum Fund, which started operations in August 2005. A FAD resident advisor to the Treasurer of the ministry finance started work in December 2006 to help strengthen expenditure management, reporting and budget execution - after a short break, a replacement has continued this work since July 2008. In 2007, a FAD TA mission provided guidance for future reform of the non-oil tax regime. In April 2009 a FAD technical assistance mission provided advice on implementation of the fiscal regimes for petroleum. In addition to PEFA and fiscal ROSC, in 2010, FAD sent follow-up mission on treasury management. Also in 2010, FAD TA mission provided advice on the calculation of estimated sustainable income and other selected petroleum fiscal issues.

LEG: A number of LEG missions have been to Timor-Leste to advise the authorities on key fiscal and financial legislation. These included tax legislation, the budget and financial management law, and banking regulations (relating to the monetary authority, the banking system, and the payments system). Jointly with MFD (now MCM), technical assistance has been provided to assist in drafting an Anti-Money Laundering and Combating the Financing of Terrorism (AML/CFT) Law. LEG has also provided assistance, in collaboration with FAD, for the drafting of the Petroleum Fund Act and tax consolidation, and in collaboration with MCM, for the drafting of the Central Bank Law.

MCM: A large number of missions took place early on to establish and develop the Central Payments Office (CPO, January 2000), which was transformed subsequently into the Banking and Payments Authority (BPA) (November 2001). Assistance was provided to establish a payments system, prepare banking legislation, and develop key functions of the monetary authority, including banking supervision, asset management, accounting, and organization and management. Assistance was also provided on the introduction of a dollar-based currency system and to issue domestic coins to supplement the use of U.S. dollar coins. More recently, assistance has been provided to (i) draft the insurance law and develop a framework for insurance supervision; (ii) strengthen banking supervision; (iii) address a broad range of issues related to AML/CFT; (iv) establish and train the investment unit responsible for managing petroleum fund assets (funded by Norway, a resident adviser commenced in March 2005 and, after a break due to contract renegotiations, a replacement continued this work from November 2008); (v) prepare for the establishment of the central bank; (vi) design of a more effective organizational structure at the BPA; and (vii) modernize payment systems, and accounting for the 
Petroleum Fund. In addition, up until 2006 long-term resident advisors, including on banking supervision, payments, and accounting, have been assigned to the BPA under a cost-sharing arrangement with the UN. A long-term resident advisor assisted the BPA General Manager from February 2005 until February 2008, with that support subsequently provided on a peripatetic basis and funded by a Japanese grant.

STA: A multi-sector statistical mission took place in November 2000 to assess the availability and quality of official macroeconomic data, and help establish the Statistic Division of the Ministry of Planning and Finance. Assistance was provided by long term statistical advisors during November 2001-October 2005. Since 2007, a peripatetic statistical advisor has provided assistance for improving data compilation and developing local capacity to prepare balance of payments statistics - responsibility for this was transferred to the BPA in 2007. Provisional annual balance of payments statements for 2006 and 2007 were published in December 2008. In early 2008 a monetary and financial statistics (MFS) mission helped introduce the standardized report forms (SRFs) for reporting monetary data to the IMF and an integrated monetary database. Subsequently, Timor-Leste has, for the first time, been able to publish a country page in IFS. A March 2009 follow-up MFS mission assisted in expanding the institutional coverage of depository corporations to include microfinance institutions and in revising the call report form that will be used to collect balance sheet data. In 2010, STA sent a follow-up mission on balance of payments statistics and a mission providing assistance on updating the CPI basket to Dili.

\section{Resident Representative}

The resident representative office closed at end-June 2009, which was established in Dili in August 2000. 


\section{ANNEX II. TIMOR-LESTE: WORLD BANK-IMF COLLABORATION}

1. The Bank and the Fund country teams for Timor-Leste, led by Mr. H. Rab (acting country manager) and Mr. W. Schule (IMF mission chief for Timor-Leste), maintain a close working relationship and have an active dialogue on a range of macroeconomic and structural issues.

2. There has been close cooperation on a range of issues including:

(i) Macroeconomic developments and economic updates: There has been close dialogue throughout the year on macro policies and economic developments. There is regular sharing of information, including Fund comments on the Bank's semiannual economic updates.

(ii) Public Finance Management: The World Bank participated in the IMF led PEFA and ROSC missions. Bank staff have been following up on the recommendations, including helping MOF put together a Strategic Plan. There is regular coordination between Bank staff on the ground and the IMF's Treasury Adviser at the Ministry of Finance.

(iii) External debt: The teams have engaged very closely on discussions around government plans to borrow. A first joint DSA was carried out during the Article IV mission in October/November 2010.

(iv) Export competitiveness study: The Bank recently completed a trade diagnostic study for which the Fund provided the analysis of external competitiveness and sustainability.

3. Based on the above, the teams agreed that Timor-Leste's main macroeconomic challenge is to manage the country's petroleum wealth effectively and sustainably to develop a stronger non-oil economy and reduce poverty. To meet this challenge Timor-Leste needs a Strategic Development Plan to help prioritize public expenditure; to strengthen public financial management; and to accelerate reforms to promote productivity and competitiveness. The effectiveness of scaling up public investment depends critically on progress in these areas.

4. Based on this shared assessment the following structural reform areas were identified as macro-critical:

- $\quad$ Strategic Development Plan: Timor-Leste envisages a rapid scaling up of public investment. The SDP should help prioritize public expenditure, promote sustainable growth and fiscal sustainability.

- Public financial management reform: The recent fiscal ROSC and PEFA missions and the Bank's review of the Planning and Financial Management Capacity Building Program found solid progress but also highlighted pressing issues around medium-term budgeting, expenditure (in particular public investment) management and procurement, and auditing. These areas are critical for the quality of expenditure. 
- $\quad$ Promoting productivity and competitiveness: Timor-Leste faces a number of challenges to promoting productivity, competitiveness and diversification in the non-oil economy. Three key priorities include addressing skills deficit; improving access to land; and strengthening the business environment, in particular access to finance and contract enforcement.

5. The teams agreed to continue the close cooperation going forward. Appendix I details the specific activities planned by the two country teams over the September 2010/2011 period. In summary, the Fund will continue to lead on macro issues, and the Bank will continue to lead PFM and macro critical structural reform issues. 
Appendix I. Timor-Leste: Bank and Fund Planned Activities in Macro-critical Areas

September 2010-August 2011

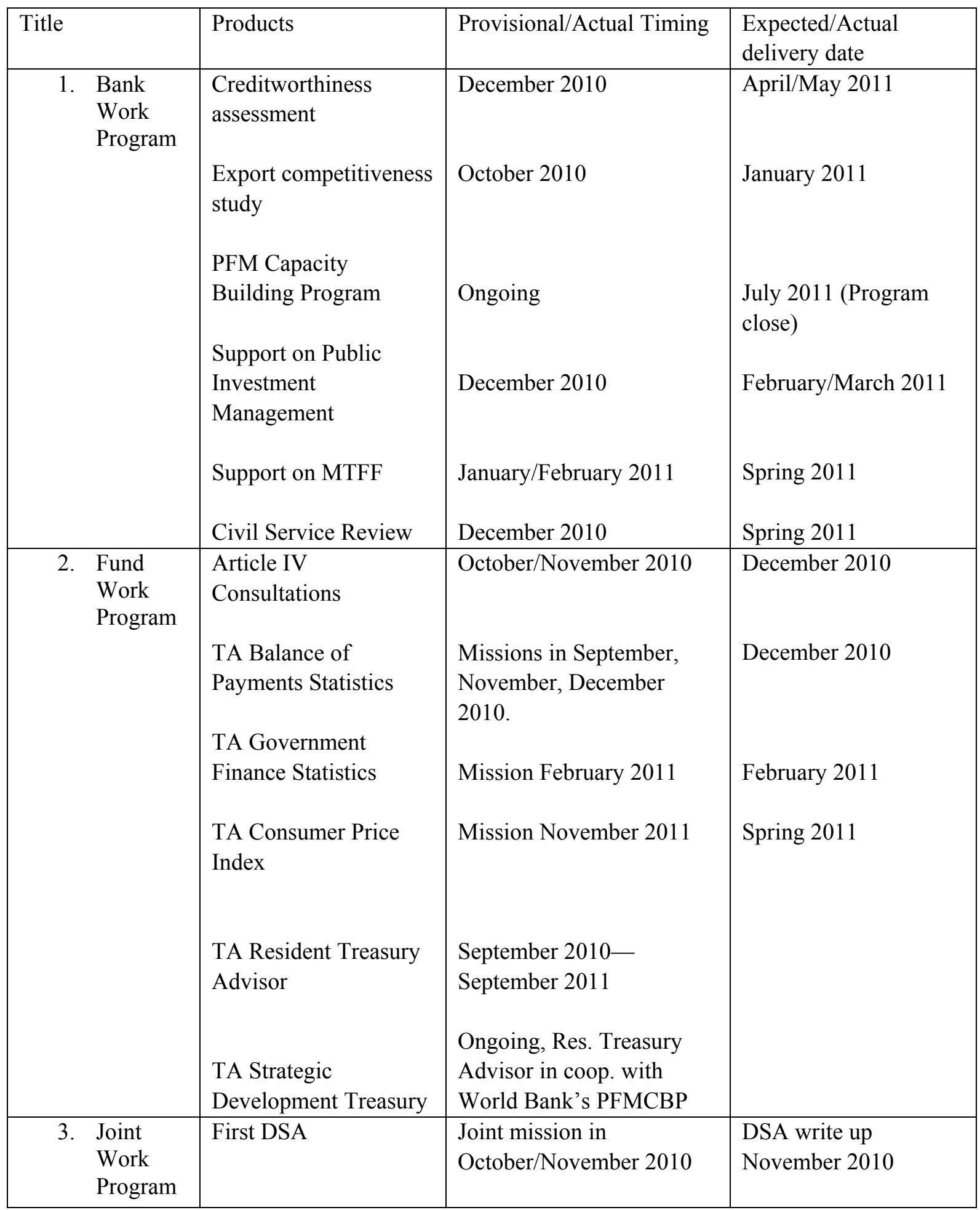




\section{ANNEX III. TIMOR-LESTE: RELATIONS WITH THE ASIAN DEVELOPMENT BANK}

(As of October 30, 2010)

Asian Development Bank (AsDB) assistance to Timor-Leste commenced in 1999. Assistance was initially conveyed through the United Nations Transitional Authority in East Timor. Timor-Leste joined the AsDB on July 24, 2002.

From 1999 to 2007, AsDB focused on the emergency rehabilitation of infrastructure, funded through the multidonor Trust Fund for East Timor (TFET). AsDB implemented about \$49 million in TFET projects covering urban and rural power, national roads, ports, and water and sanitation. \$4 million in TFET funds were used to establish and capitalize a microfinance institute.

AsDB's recent operations have been guided by the Country Program and Strategy Update 2006-2008 and the Country Operations Business Plans 2008-2011. ${ }^{1}$ AsDB support has been closely aligned with the Government's National Development Plan and its goals to improve physical infrastructure and build capacity to manage and deliver quality basic social services.

The Asian Development Fund (ADF) program commenced in 2005. \$62 million in ADF grant funding has been approved, focused on essential infrastructure. A national roads rehabilitation project ( $\$ 10.0$ million ADF) concluded in 2009 and has been succeeded by a new Road Network Development Sector Project (\$46.0 million ADF). AsDB administers a complementary \$3 million project in support of road side communities, funded by the Japan Fund for Poverty Reduction. AsDB also supports the Dili water supply and sanitation project ( $\$ 6.0$ million ADF).

Timor-Leste is the recipient of AsDB's largest ever technical assistance (TA) grant, the Infrastructure Project Management TA. This $\$ 15$ million package, funded with AusAID co-financing, commenced in 2007 and helps plan, prepare, and execute the Government's capital development program. Other AsDB activities include the strengthening of microfinance, statistics and macroeconomic management, and a civil service training institute.

To-date ADB has approved 9 grants ( 6 from the TFET and 3 from the ADF) for a total amount of $\$ 114.8$ million, and 30 technical assistance (TA) projects for a total of $\$ 28.3$ million. Three grant funded projects ( $\$ 52.0$ million) and 4 TA projects ( $\$ 17.1$ million) are active.

There has been no lending to Timor-Leste, with all ADF resources provided as grants. ADF resources are set to move from 100\% grant in 2010 to $67 \%$ grant and 33\% loan in 2011-2012 and then to 33\% grant and 67\% loan in 2013-2014. Timor-Leste's ADF Allocation for 2011-2012 is \$30.2 million.

\footnotetext{
${ }^{1}$ AsDB. 2005. Country Strategy and Program Update 2006-2008: Democratic Republic of Timor-Leste, and ADB, 2007. Country Operations Business Plan for Timor-Leste 2008-2010.
} 


\section{ANNEX IV. TIMOR-LESTE: STATISTICAL ISSUES}

As of October 27, 2010

\section{Assessment of Data Adequacy for Surveillance}

General: Data provision to the Fund has serious shortcomings that significantly hamper surveillance. Weaknesses remain in the macroeconomic data, especially the national accounts. A multi-sector Fund statistics mission visited Dili in November 2000 and assisted the authorities in establishing objectives for restoring economic statistics and in setting up a Statistical Division in the Ministry of Planning and Finance in 2001. A long-term resident statistical advisor assisted the Statistical Division during November 2001-February 2004, and was succeeded by a new resident advisor until September 2005. Technical assistance in balance of payments (peripatetic) and monetary statistics to the Banking and Payments Authority (BPA) began in mid 2007 and January 2008, respectively, and will continue through at least FY 2010.

National Accounts: Under a World Bank-financed project, national accounts data for 2000 (both sector and expenditure basis) were estimated by the Boston Institute for Developing Economies (BIDE). In late 2004, BIDE, under a follow-up World Bank project, produced GDP estimates for 2001-03 that suffer from limited source data and substantial extrapolation. The Statistics Directorate has not been able to replicate the BIDE estimation exercise. Since April 2009, the authorities have been employing a World Bank funded consultant to develop annual estimates of GDP.For the first time in 2010, estimates of GDP (including for the oil sector) have been prepared by the Macroeconomic Unit in the Ministry of Finance in cooperation with Fund staff. To improve national accounts data, appropriate staff and substantial efforts in data collection and compilation are needed.

Price statistics: A consumer price index (CPI) has been compiled for Dili since December 2001 and for the whole country since June 2003. Prices for all items in the basket are collected on a monthly basis in the Dili region. Elsewhere, prices are collected at quarterly intervals for a representative selection of other regions throughout Timor-Leste. The measure of the interim monthly price movements for each item outside the Dili region is then estimated using the corresponding Dili region item price movement as an indicator series. To address quality weaknesses, in particular inappropriate and outdated weights, the commodity basket and the weighting scheme were revised in mid-2003 under a donor-supported project. A technical assistance mission during November 8-19, 2010 will assist in rebasing the CPI using data derived from the Timor-Leste Living Standards Survey that was conducted in 2006-7. Shortcomings in data collection need to be addressed and products and outlets updated.

Labor market statistics: Annual data on public sector employment and wages are available in official budget documentation. No official data are compiled regularly on private sector employment and wages, except for partial data from various household surveys conducted by UNDP, ILO, ADB, and the World Bank. In 2010, the Macroeconomic Unit in the Ministry of Finance has started to conduct wage surveys.

Government finance statistics: Official data on central government revenue and expenditure are published quarterly. Audited annual financial statements of the executed budget are made available to APD as soon as they are released, normally three months after the end of the fiscal year. In addition, 
monthly data are available on request through the budget, treasury, and revenue services. The compilation of revenue and expenditure follows the classification of the Manual on Government Finance Statistics (GFSM 1986) (including expenditure based on both functional and economic classification). Only partial data are available on fiscal and quasi-fiscal activities undertaken by bilateral donors outside the central government budget. Currently, there are four non-financial public enterprises, which are treated as government agencies and data on their operations are available in the audited annual financial statements. No data are currently reported for the GFS Yearbook and the International Financial Statistics. A technical assistance mission is tentatively scheduled to the last quarter of FY 2011. This mission will aim to analyze the public sector's composition and accounting information as well as to set up a system to regularly report GFSM2001 data to users, including the IMF.

Monetary statistics: Monetary statistics are compiled by the BPA, generally following the methodology of the Monetary and Financial Statistics Manual. However, data are incomplete because of the absence of official data on public currency holdings - which are difficult to compile under the current currency regime - and of banks' positions with public nonfinancial corporations. The BPA publishes current monetary data on its website on a regular monthly basis, as well as a quarterly bulletin and an annual report with key monetary aggregates.

Detailed monthly monetary data are available from July 2002 onward following the introduction of the standardized report forms (SRFs). Beginning in February 2008, monetary data have been reported to the IMF for publication in the International Financial Statistics. The BPA is planning to introduce a new chart of accounts for the BPA in the coming months and a revised call report form (CRF) in late 2010 to obtain balance sheet information from other depository corporations (ODCs). A March 2010 mission assisted the authorities in finalizing the design of the CRF and derived the SRF for the ODCs based on this revised CRF. A follow-up mission could be scheduled for FY 2012 to assist the authorities in introducing the SRFs for the central bank based on the new chart of accounts of the BPA and for the ODCs based on the revised CRF and including the credit unions in the institutional coverage of the ODCs. Additionally, a new CRF could be designed to collect balance sheet data from insurance companies, and surveys compiled for financial corporations.

Balance of payments: Monthly data on merchandise exports and imports have been produced starting March 2000, based on customs reports with a breakdown for major commodity categories. A major data gap is the significant underestimation of imports of goods. Also not included are oil and gas exports; an estimate of the large, unrecorded border trade; and some goods exempt from customs duties. Further, customs data may underestimate imports because of undervaluation of declared goods. Monthly merchandise trade data are now published regularly but there are significant gaps in the series for 2006 and 2007. In the Fund staff estimates, data on oil/gas revenue are currently recorded as current income based on fiscal data.

With technical assistance provided by the IMF's Statistics Department, annual balance of payments data for 2006 and 2007 were published by the BPA in December 2008. Data are now available for 2006-2009.

While methodology for the production of basic annual estimates of the balance of payments statistics are in place, further development work is anticipated to address limitations of existing data sources, in particular grants, merchandise trade statistics, and travel exports. The coverage of the survey of 
foreign direct investment enterprises needs to be expanded, and the exploration and extraction activities, in the Joint Petroleum Development Area and in the exclusive territory, require ongoing monitoring. As the economy grows, it will be important to monitor new activities such as new direct investment. Currently, there is limited information on remittances from Timorese working abroad and improvement in the estimation/compilation procedures of such remittances should be pursued.

Some data on stocks of financial assets and liabilities have been collected as the balance of payments development has taken place, and the annual international investment position statements should be finalized. Development of quarterly statistics should also be pursued.

The next balance of payments technical assistance mission will be conducted in November 2010 .

\section{Data Standards and Quality}

Not a General Data Dissemination System

No data ROSC is available.

participant. 


\section{Timor-Leste: Table of Common Indicators Required for Surveillance}

(As of November 01, 2010)

\begin{tabular}{|c|c|c|c|c|c|}
\hline & $\begin{array}{c}\text { Date of } \\
\text { latest } \\
\text { observatio } \\
\text { n }\end{array}$ & $\begin{array}{c}\text { Date } \\
\text { receive } \\
\mathrm{d}\end{array}$ & $\begin{array}{c}\text { Frequenc } \\
y \text { of } \\
\text { Data }^{6}\end{array}$ & $\begin{array}{c}\text { Frequency } \\
\text { of } \\
\text { Reporting } \\
6\end{array}$ & $\begin{array}{c}\text { Frequency } \\
\text { of } \\
\text { publication } \\
6\end{array}$ \\
\hline Exchange Rates & $11 / 2010$ & $11 / 2010$ & $\mathrm{D}$ & $\mathrm{D}$ & $\mathrm{D}$ \\
\hline $\begin{array}{l}\text { International Reserve Assets and Reserve Liabilities of } \\
\text { the Monetary Authorities }{ }^{1}\end{array}$ & $9 / 2010$ & $10 / 2010$ & M & M & M \\
\hline Reserve/Base Money (excludes currency in circulation) & $9 / 2010$ & $10 / 2010$ & M & M & M \\
\hline Broad Money (excludes currency in circulation) & $9 / 2010$ & $10 / 2010$ & M & M & M \\
\hline Central Bank Balance Sheet & $9 / 2010$ & $10 / 2010$ & M & M & M \\
\hline Consolidated Balance Sheet of the Banking System & $9 / 2010$ & $10 / 2010$ & M & M & M \\
\hline Interest Rates ${ }^{2}$ & $9 / 2010$ & $10 / 2010$ & M & M & M \\
\hline Consumer Price Index & $6 / 2010$ & $7 / 2010$ & Q & Q & Q \\
\hline $\begin{array}{l}\text { Revenue, Expenditure, Balance and Composition of } \\
\text { Financing }{ }^{3}-\text { General Government }^{4}\end{array}$ & $\cdots$ & $\cdots$ & $\cdots$ & $\cdots$ & $\cdots$ \\
\hline $\begin{array}{l}\text { Revenue, Expenditure, Balance and Composition of } \\
\text { Financing }{ }^{3} \text { - Central Government }\end{array}$ & $12 / 2009$ & $9 / 2010$ & A & A & A \\
\hline $\begin{array}{l}\text { Stocks of Central Government and } \\
\text { Central Government-Guaranteed Debt }\end{array}$ & & & o debt cont & cted & \\
\hline External Current Account Balance & 2009 & $8 / 2010$ & A & A & A \\
\hline Exports and Imports of Goods and Services ${ }^{5}$ & $8 / 2010$ & $9 / 2010$ & M & M & $\mathrm{M}$ \\
\hline GDP/GNI & 2009 & $9 / 2010$ & $\ldots$ & $\ldots$ & $\ldots$ \\
\hline Gross External Debt & \multicolumn{5}{|c|}{ Not applicable } \\
\hline International Investment Position ${ }^{7}$ & \multicolumn{5}{|c|}{ Not available } \\
\hline
\end{tabular}

\footnotetext{
${ }^{1}$ Includes reserve assets pledged or otherwise encumbered as well as net derivative positions.

${ }^{2}$ Both market-based and officially-determined, including discount rates, money market rates, rates on treasury bills, notes and bonds.

${ }^{3}$ Foreign, domestic bank, and domestic nonbank financing.

${ }^{4}$ The general government consists of the central government (budgetary funds, extra budgetary funds, and social security funds) and state and local governments. For Timor-Leste it includes public spending from donors, on which regular data on execution are not available.

${ }^{5}$ Includes only goods. There are significant gaps in the series. No information on services is available.

${ }^{6}$ Daily (D), Weekly (W), Monthly (M), Quarterly (Q), Annually (A); NA: Not Available.

${ }^{7}$ Includes external gross financial asset and liability positions vis-à-vis nonresidents.
} 


\title{
INTERNATIONAL DEVELOPMENT ASSOCIATION AND \\ INTERNATIONAL MONETARY FUND
}

\section{DEMOCRATIC REPUBLIC OF TIMOR-LESTE}

\section{Joint World Bank/IMF 2010 Debt Sustainability Analysis}

\author{
Prepared by the Staffs of the International Development Association and \\ the International Monetary Fund \\ Approved by Ray Brooks and Dhaneshwar Ghura (IMF) \\ and Carlos A. Primo Braga and Tunc Tahsin Uyanik (World Bank)
}

December 3, 2010

The medium-term fiscal path for Timor-Leste is subject to uncertainties regarding spending commitments and income prospects from petroleum, which complicates the Debt Sustainability Analysis (DSA). The baseline macroeconomic scenario assumes a significant scaling-up of public spending, future petroleum income only from fields with approved development plans, and a moderate borrowing envelope. Under this scenario, the external low income country DSA indicates a low risk of debt distress. The public DSA suggests that overall public sector debt dynamics are sustainable in light of a gradual approach of moderate borrowing and substantial savings in the PF.

\section{INTRODUCTION}

\section{A young nation with a high incidence of poverty, Timor-Leste has been heavily} dependent on petroleum resources since the start of offshore production in 2004. Proven reserves or production levels stand out in comparison to the non-oil economy. However, they neither make Timor-Leste a major petroleum exporter in the world nor a rich country in per capita income terms. By 2009, petroleum revenue accounted for almost 80 percent of GNI and withdrawals from the Petroleum Fund for about 95 percent of total government revenue (including grants). The Petroleum Fund (PF), established in 2005, is the cornerstone of petroleum resource management and its assets reached $\$ 6.5$ billion or ten times the non-oil GDP in August 2010, which are invested entirely abroad.

2. The first joint DSA for Timor-Leste is conducted amid the authorities' quest for double-digit non-oil GDP growth in the next two decades by scaling up public

investment. Currently, Timor-Leste has no public or external debt and withdrawals from the $\mathrm{PF}$ have been financing non-oil fiscal deficits. But the government is contemplating external borrowing, as a way to substitute withdrawals from the $\mathrm{PF}$, to meet pressing development needs. Loans from development partners may come with technical assistance, which would be instrumental in strengthening the country's policy and institutional capacity, and in 
particular play an instrumental role for the government's planned complex infrastructure investments. At present, Timor-Leste has a weak Country Policy and Institutional Assessment (CPIA) performance rating, corresponding to the lowest set of indicative debt thresholds.

3. The authorities' agreed broadly with the DSA assessment. Given PF resources, there is no immediate financial need for borrowing. However, the authorities see moderate and gradual borrowing as a way to access technical assistance from development partners in particular to address capacity constraints in the appraisal, selection, and implementation of infrastructure projects. The authorities also see borrowing as a way to diversify sources of financing and help build debt management capacity from scratch.

\section{UNDERLYING DSA ASSUMPTIONS}

4. The first decade of Timor-Leste's independence saw a significant rise in national income, accompanied by large volatility associated with petroleum income. Thanks to petroleum income, real GNI grew at an annual average of 27 percent, but ranged from a contraction of 25 percent in 2009 to an expansion of over 50 percent each in 2007-08. Nonoil GDP expanded over the decade at an annual average rate of 5 percent with large volatility (standard deviation of 8), reflecting Timor-Leste's post-conflict status.

\section{The DSA takes account of both the non-oil economy and income from}

petroleum. Currently GDP does not include petroleum production that takes place in the joint production area between Timor-Leste and Australia. However, based on Timor-Leste's very small non-oil economy alone, any meaningful borrowing would be unsustainable. Therefore, to reflect the special feature of the economy, the following adjustments are made in measuring Timor-Leste's repayment capacity: (i) GNI is used to capture the size of the wider economy; (ii) wider exports cover exports of non-oil goods and services plus oilrelated income, including oil exports (recorded as oil income in the balance of payments) and the PF investment income net of interest payments abroad; and (iii) total public sector revenue equals non-oil revenue plus estimated sustainable income (ESI) from petroleum (Box 1). In essence, Timor-Leste's borrowing capacity depends crucially on petroleum exports, the accumulation of $\mathrm{PF}$ assets, and growth of the non-oil economy.

\footnotetext{
${ }^{1}$ GNI consists of non-oil GDP and total net income from abroad as recorded in the balance of payments. Unless otherwise indicated, GDP throughout the paper (including tables and charts) refers to GNI.
} 


\section{BoX 1. The Estimated Sustainable InCOME}

- $\quad$ The Petroleum Fund (PF) Law defines the estimated sustainable income (ESI) as 3 percent of Timor-Leste's total petroleum wealth, including the current PF balance plus the net present value of future petroleum receipts.

- Rather than a rigid fiscal ceiling, the ESI is a benchmark to guide budget withdrawals from the PF and inform policy discussions. Actual withdrawals can exceed the ESI as long as the government delivers a detailed explanation to parliament why doing so is in the long-run interest of country.

- The ESI is calculated every year based on a set of assumptions on petroleum output and prices. The PF Law requires prudent assumptions to be used, as 70 percent of TimorLeste's oil wealth remains under the sea and there is no diversification across multiple projects.

- Only projects with an approved development plan and firm investment commitment (i.e., the Bayu Undan and Kitan fields) have been included in the ESI calculation. The Greater Sunrise field has been declared a commercial discovery, but does not have an approved development plan, and therefore according to current policy should not be included in ESI. Further, its exclusion appears justified given the material uncertainties surrounding the technical and commercial configuration of the project and the potential for significant delays. However, Sunrise is a confirmed petroleum resource and its exclusion from ESI arguably puts some strain on the credibility of the policy.

- Nevertheless, the Ministry of Finance should begin evaluating its potential effect on petroleum wealth and fiscal financing. Perceived petroleum wealth from Sunrise is already informing wider thinking in government and civil society about sustainable spending levels, and quantification of the potential revenue flows would help to inform and guide that discussion. In particular, this exercise would allow the authorities to manage expectations and encourage a more realistic assessment of the potential addition to petroleum wealth from Sunrise.

- Until 2010, the oil price used for ESI calculation has been the "low" case from the U.S. Energy Information Administration (EIA) Annual Energy Outlook. To address the concern within the government that the low case lies materially below market and other respected forecasters expected prices, the authorities recently explored other alternative methodologies for determining a price forecast more reflective of expectations but still prudent. It came to a conclusion of using the average of the EIA low and reference cases, which balances between being realistic and prudent. For 2011, the average price generates an ESI of \$734 million compared to \$548 million under the EIA low case price. 
6. The DSA is based on the macroeconomic framework discussed with the authorities during the 2010 Article IV Consultations (Box 2). The baseline takes account of the authorities' current policy intentions, which include a significant and front-loaded scaling-up of public investment during the next decade. Financing of the scaling-up is to come from higher withdrawals from the PF, some of which can be substituted by external borrowing. It was also assumed that no new petroleum fields come on stream, such as the Greater Sunrise field, which has a promising potential, but its development plans are currently under negotiation.

\section{Box 2. Baseline Macroeconomic and Petroleum Sector Assumptions (2011-30)}

Real sector: real non-oil GDP is projected to grow at close to 9 percent on average over the medium term before gradually reverting to its potential of 6 percent in the long run, slightly above the historical average of 5 percent during 2002-09. Due to a lapse of one-off factors, such as the agricultural rebound from the 2007 drought, as well as bad weather in 2010 , staff projects non-oil GDP growth to decelerate from an annual average rate of 11 percent during 2007-09 to about 6-7 percent in 2010-11. Medium-term projections reflect staff's assessment that Timor-Leste's weak policy and institutional capacity will undermine the effectiveness of significant front-loaded public investment scaling-up. As a result, medium-term growth is projected at a rate lower than that being contemplated by the authorities. Given the economy's limited absorptive capacity, inflation is expected to remain high at 6 percent throughout the medium-term before declining gradually to 4 percent in the long run.

Fiscal sector: tax revenue is projected to stay broadly stable at $7 \frac{1}{2}-81 / 2$ percent of non-oil GDP. Reflecting front-loaded scaling-up, capital expenditure rises from an average of 33 percent of non-oil GDP in 2009-10 to an average of 54 percent during 2011-15 before declining gradually to 20 percent in the long-run. Despite the government's plans to keep noncapital spending growth in line with inflation growth, staff projects current expenditure to average at 68 percent of non-oil GDP during 2011-15 before declining gradually to about 30 percent by 2030 . Unlike the authorities, staff projections include recurrent cost associated with large public investment. Consequently, staff expects the non-oil fiscal deficit to rise from 92 percent of non-oil GDP in 2009 to an average of 105 percent during 2010-15 before declining to 34 percent in 2030. Financing of prolonged large non-oil deficits will require withdrawals from the PF consistently above the ESI.

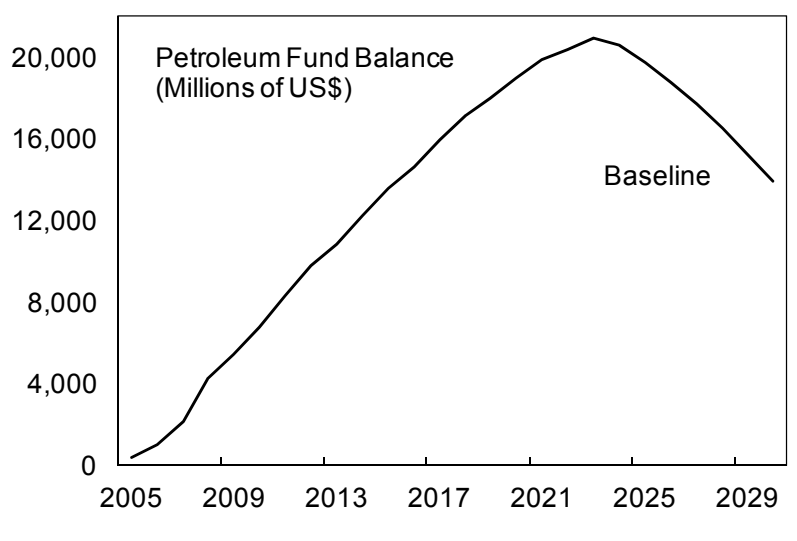

External sector: staff projects exports of non-oil goods and services to grow at an average of about 12 percent annually. However, declining petroleum income will worsen the current 
account balance from a surplus of 245 percent of non-oil GDP in 2009 to a deficit of 26 percent of non-oil GDP by 2024. Current account deficits will be financed largely by withdrawals from the PF.

External financing: for this DSA, it is assumed that Timor-Leste will borrow around \$900 million during 2011-30 from bilateral and multilateral donors to fund its development program. Around 40 percent of the loans will be on highly concessional terms. Concessional loans are projected to wind down because Timor-Leste's per capita GNI is already above the threshold for Low Income Countries that are eligible for highly concessional loans from several institutions. Other loans are assumed to carry a 5 year grace period, 25 year maturity, and an interest rate of $4 \frac{1}{2}$ percent. Eligibility for such loans will be subject to creditworthiness and other assessments depending on the financier. The assumptions are based on very preliminary indications on potential lending and are therefore subject to change.

The financing assumptions in the DSA are driven mostly by Timor-Leste's changing status from Low Income to Middle Income Country, which has implications for access to highly concessional resources. To date Timor-Leste has received only grants from the IFIs (World Bank and Asian Development Bank) on an exceptional basis because of its post-conflict status and fledgling institutions. Ordinarily, as a Low Income Country, Timor-Leste would have received highly concessional credits because the country is not at risk of debt distress. This is likely to be the case from next year. In recent years, however, Timor-Leste's per capita GNI has gone above the eligibility threshold even for highly concessional credits because of the rise in petroleum receipts. The DSA therefore assumes that Timor-Leste will no longer be eligible for highly concessional loans over the medium-term, because the country's GNI per capita makes it a (lower) Middle Income Country.

Petroleum production: projections are based on two active fields, Bayu Undan and the relatively small Kitan field. It is expected that Bayu Undan will cease production by 2025 and Kitan by 2017. Total production is expected to peak at close to 70 million barrels of oil equivalent in year 2012 before winding down by 2025 .

Petroleum price: projections are based on an average of EIA's low case and reference case prices, published in May 2010. Except for a 10 percent decline in 2011 from $\$ 76$ to $\$ 68$ per barrel, crude oil prices are expected to rise quite smoothly at an average rate of between 3 and 4 percent per year in nominal terms.

7. The long-term impact of the rapid scale up in public investment on growth will depend critically on the prioritization and quality of investment. Since 2006/07, capital investment managed and financed by the government has increased by nearly tenfold. All of it was channeled through the national budget. The rapid scale up, however, has stretched the limited investment management capacity of the public and the private sector with negative implications for the quality of projects, their implementation, and ex post monitoring. To 
improve cost and quality control, the government is planning to establish an Infrastructure Fund and a new agency (ADN) that will be responsible for project appraisal, design, and monitoring. Procurement for large projects (more than $\$ 1$ million) will be managed by a Procurement Technical Secretariat. The government intends to staff these new agencies with well qualified professionals and is also looking at different outsourcing options.

\section{The impact of investment on growth will also depend on the quality of}

complementary policies and institutions. ${ }^{2}$ These will affect the ability of the private sector to take advantage of infrastructure development. However, the private sector remains small, relative to the size of planned public sector investment. Timor-Leste also has a very challenging business environment. ${ }^{3}$ Key priorities include simplifying business start up, strengthening contract enforcement and increasing access to finance. Other structural reform priorities include investment in skills in the rapidly growing labor market, improving access to land, ensuring access to energy, and liberalization of the telecommunications sector. Finally, public finance management reforms will also play a major role in promoting investment and growth. ${ }^{4}$ The government is taking steps in all these areas.

\section{EXTERNAL DSA}

\section{Baseline Scenario}

9. The baseline scenario indicates that all debt stock and debt service indicators are below their policy-dependent indicative thresholds throughout the projection period (Figure 1 and Table 1). Specifically, the PV of debt-to-GDP ratio and the PV of debt-torevenue ratio are expected to peak at 17 percent and 68 percent respectively by 2025 , much below their thresholds of 30 percent and 200 percent, respectively. The PV of debt-to-exports ratio is projected to peak at 67 percent in 2026, below the threshold of 100 percent. The two debt service ratios are projected to rise over time but remain considerably below their thresholds.

\section{Standard Alternative Scenarios and Stress Tests}

\section{Stress tests were conducted to assess the robustness of the baseline scenario to} various shocks. The results are presented in Table 2 and illustrated in Figure 1. Notably, only a shock of lower export growth during 2011-12, which can be interpreted as a shock to

\footnotetext{
${ }^{2}$ IMF and World Bank, A Review of Some Aspects of the Low-Income Country Debt Sustainability Framework, August 2009; and Era Dabla-Norris and Felipe Zanna, The Investment and Growth Nexus, IMF, 2010.

${ }^{3}$ IFC and World Bank, Doing Business 2011, November 2010: Timor-Leste ranks 174 out of 183 countries in the overall Ease of Doing Business.

${ }^{4}$ IMF, 2010 Report on the Observance of Standards and Codes (ROSC) Fiscal Transparency Module and Public Financial Management-Performance Report for Timor-Leste.
} 
petroleum production or prices given the dominance of petroleum exports, would result in some ratios breaching thresholds. Specifically,

- Two debt stock ratios are expected to breach their indicative thresholds under a very large shock to exports (B2) generated by the standard DSA template. The PV of debt-to-GDP ratio is projected to peak at 37 percent and breach its threshold during 2018-27, and the PV of debt-to-exports ratio is expected to breach its indicative thresholds from 2024 when oil income winds down.

- $\quad$ All debt ratios remain below their thresholds under standard shocks to GNI growth, deflator, and non-debt creating flows (B1, B3, and B4).

- The stress test of a combination of one-half standard deviation shocks (B5) leads to more favorable debt dynamics. However, deducting one-half standard deviation from large historical averages is unrealistic and leads to overly optimistic assumptions on growth of GNI and exports during 2011-12.

- The historical scenario (A1) indicates extremely favorable debt dynamics relative to the baseline. This is because the baseline scenario expects that petroleum income will steadily wind down by 2025 while the historical scenario applies a counterfactual continuation of large petroleum income from the Bayu Undan field throughout the projection period.

- Under the scenario of less favorable loan terms (A2), all debt burden and debt services indicators worsen relative to the baseline. But the ratios remain below their indicative thresholds throughout the projection period.

- The stress test of a one-time 30 percent depreciation is not applicable due to official dollarization.

\section{Customized Export Shock}

11. The standard DSA stress tests do not take account of Timor-Leste's special situation as a young post-conflict country. These tests are based on historical volatility of the last eight years, which is particularly high and therefore may not be representative. Specifically, the standard export shock would imply an unrealistic negative shock of about 140 percent. Given the substantial reliance on oil, an appropriate size of the export shock would be one that mimics a realistic oil price shock of 30-40 percent during the last 10 years.

12. Under a customized export shock of 60 percent to the baseline, Timor-Leste is found to be at low risk of debt distress. This size of shock represents a worst case scenario because it almost doubles the typical oil price shock observed in the last 10 years. As shown in Figure 1 and Table 3, all debt stock and debt service ratios are projected to remain under their indicative thresholds throughout the projection period (B6). 


\section{Public DSA}

13. With only external borrowing by the government, the results of public debt DSA mirror the external debt DSA. In light of substantial asset accumulation in the PF, TimorLeste's public debt DSA is conducted on a net debt basis (i.e., gross public debt minus the PF assets). Under the baseline scenario (Table 3 and Figure 2), all three debt stock and debt service ratios are projected to rise eventually but stay well within their indicative thresholds.

\section{Timor-Leste's net public debt does not appear to be vulnerable to the} standardized DSA stress tests (Figure 2 and Table 4). This is because the buildup of substantial PF assets provides a significant cushion against shocks. However, Timor-Leste's net public debt is projected to increase significantly around 2025 when oil income winds down. This highlights the importance of keeping borrowing envelope limited and being fiscally prudent with spending from the PF.

\section{Conclusions}

15. With a moderate borrowing envelope, Timor-Leste is at low risk of debt distress. All debt stock and debt service ratios are projected to stay below their indicative thresholds under baseline and stress tests. The public DSA also suggests that Timor-Leste's overall public sector debt dynamics are sustainable with moderate borrowing. Nevertheless, a range of sensitivity analysis reveals Timor-Leste's vulnerability to shocks, particularly those related to petroleum production and prices, reflecting the country's heavy dependence on one single commodity (petroleum). Therefore, to minimize the risk of debt distress, it is advisable for Timor-Leste to take a gradual and moderate approach to borrowing from development partners and be fiscally prudent.

\section{A key challenge for Timor-Leste is to use its petroleum wealth effectively and} sustainably to develop non-oil economy. Given the flexibility embedded in the PF Law and the calculations of the ESI, a major constraint on boosting growth through scaling-up public investment comes from weak policy and institutional capacity rather than from the availability of financing. Therefore, an appropriate level of public expenditure should be commensurate with the government's capacity to plan, implement, and review investment projects, and with the ability of private sector producers to take advantage of public capital. Close cooperation with the World Bank, the Asian Development Bank, and other development partners would help prioritizing, sequencing, and managing expenditures. The decision of how to finance development projects - through borrowing or more withdrawals from the PF-is of second order. A gradual approach to borrowing is advisable to allow time for building up debt management capacity from scratch. Moreover, preference should be given to concessional loans rather than commercial loans to contain the risk of debt distress. 
Figure 1. Timor-Leste: Indicators of Public and Publicly Guaranteed External Debt under Alternatives Scenarios, 2010-2030 1/

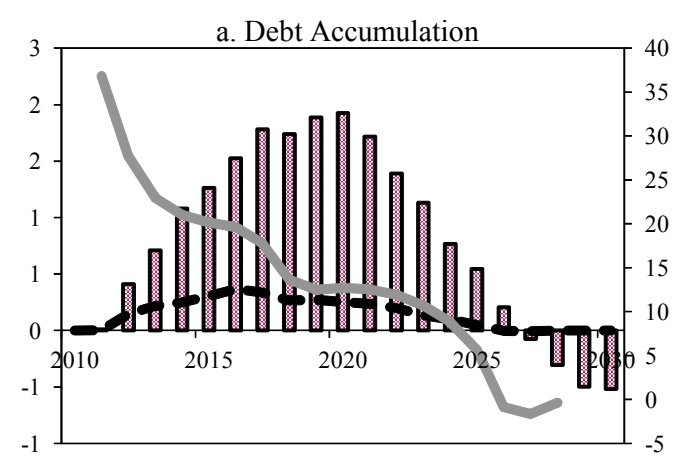

Rate of Debt Accumulation

---.-- Grant-equivalent financing (\% of GDP)

Grant element of new borrowing (\% right scale)
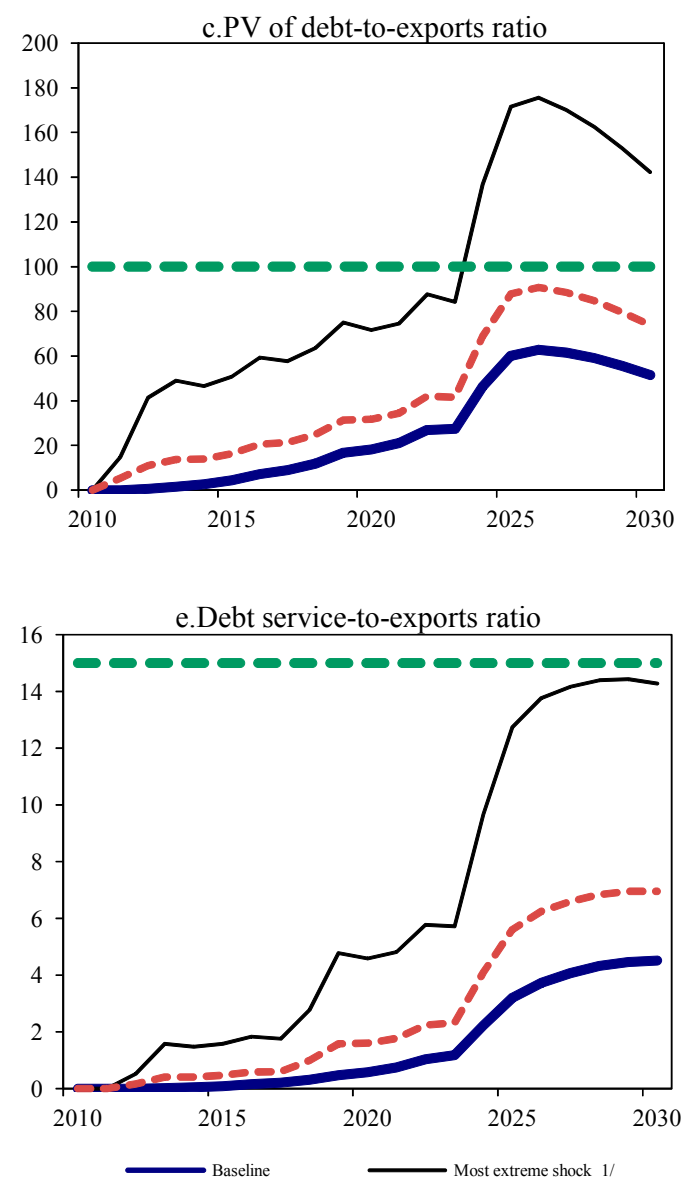
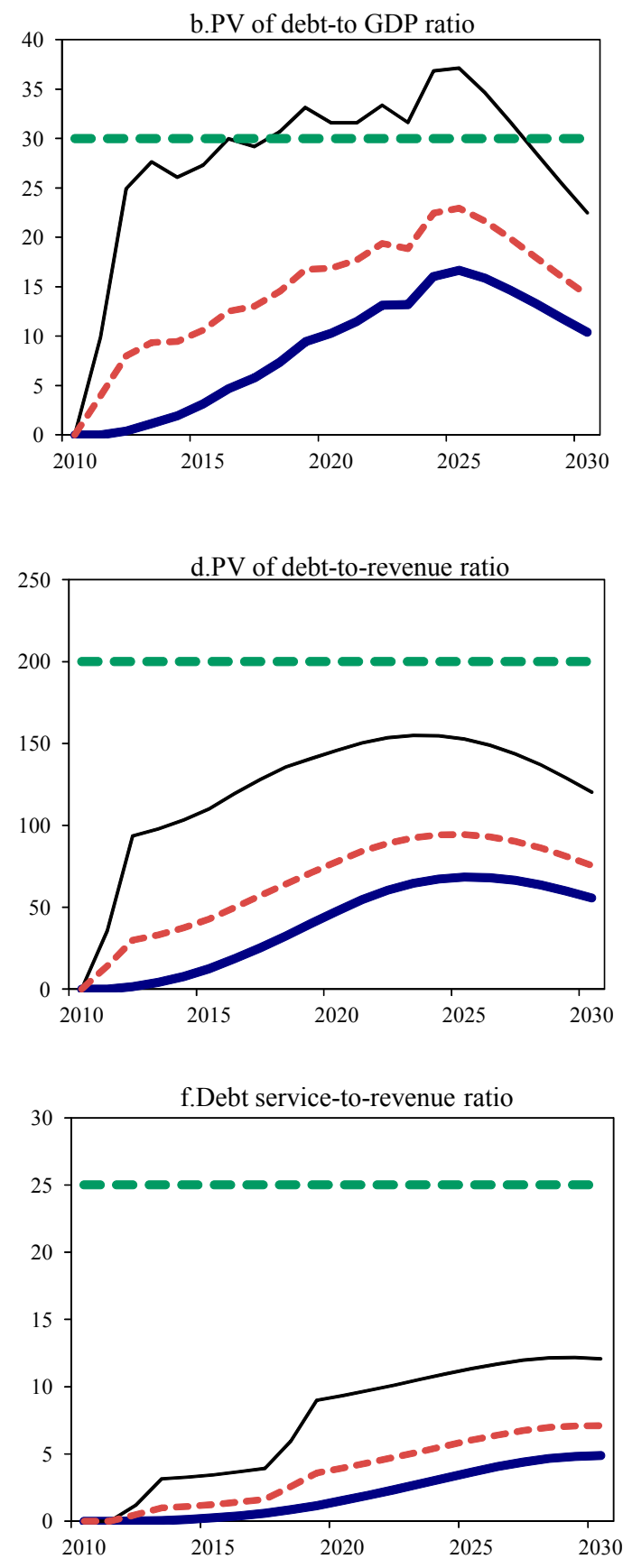

- - - Threshold $\quad-\infty-\infty$ customized export shock

Sources: Country authorities; and staff estimates and projections.

1/ The most extreme stress test is the test that yields the highest ratio in 2020. In figure b. it corresponds to a Exports shock; in c. to a Exports shock; in d. to a Exports shock; in e. to a Exports shock and in figure f. to a Exports shock. 
Figure 2.Timor-Leste: Indicators of Public Debt Under Alternative Scenarios, 2010-2030 1/
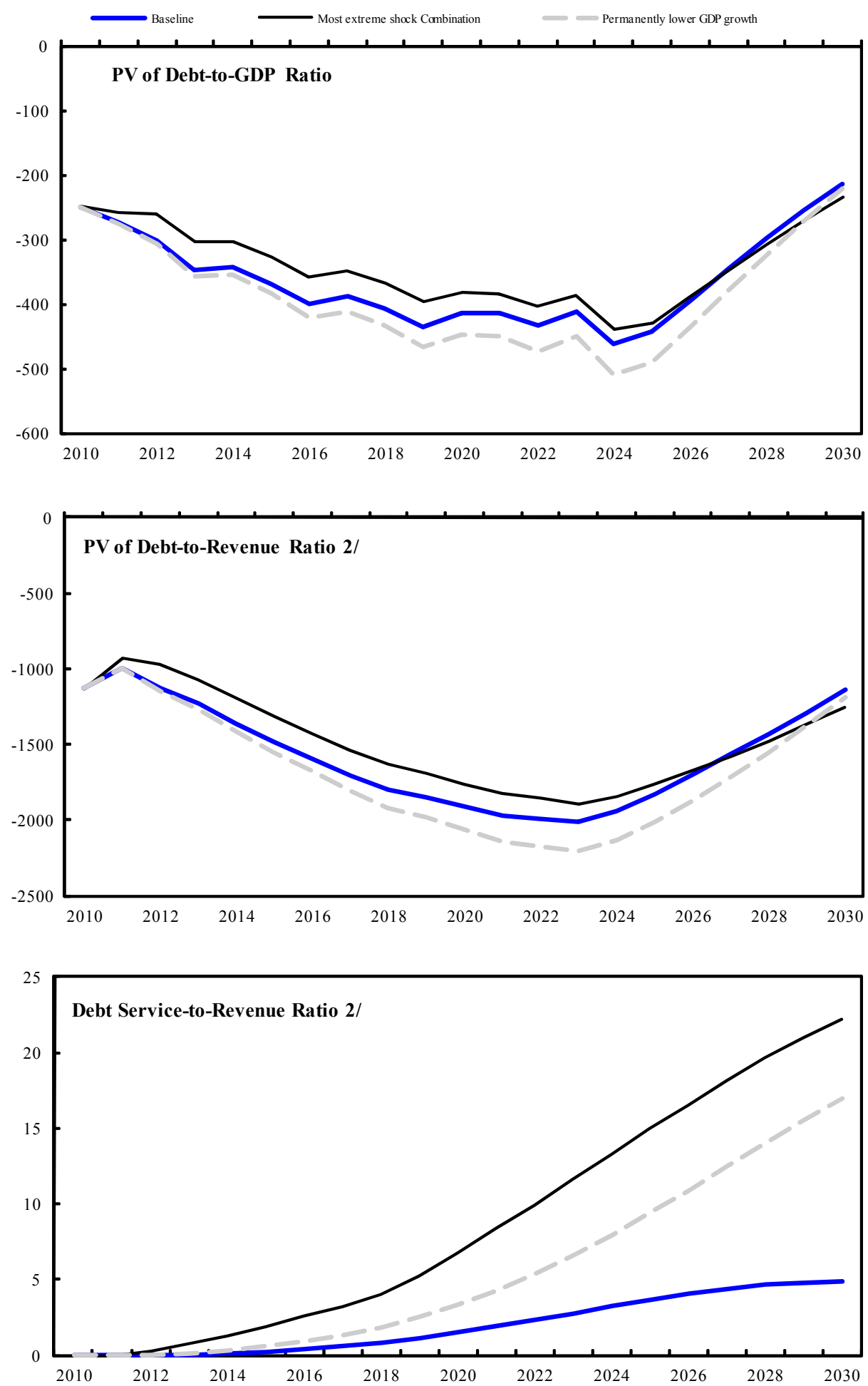

Sources: Country authorities; and staff estimates and projections.

$1 /$ The most extreme stress test is the test that yields the highest ratio in 2020.

2/ Revenues are defined inclusive of grants. 
Table 1.: External Debt Sustainability Framework, Baseline Scenario, 2007-2030 1/ (In percent of GDP, unless otherwise indicated)

\begin{tabular}{|c|c|c|c|c|c|c|c|c|c|c|c|c|c|c|c|}
\hline & \multicolumn{3}{|c|}{ Actual } & \multirow{2}{*}{\multicolumn{2}{|c|}{ 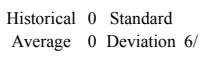 }} & \multicolumn{6}{|c|}{ Projections } & \multirow{2}{*}{\multicolumn{3}{|c|}{$2010-2015$}} & \multirow{2}{*}{$\begin{array}{c}2016-2030 \\
\text { Average }\end{array}$} \\
\hline & 2007 & 2008 & 2009 & & & 2010 & 2011 & 2012 & 2013 & 2014 & 2015 & & & & \\
\hline External debt (nominal) 1/ & 0.0 & 0.0 & 0.0 & & & 0.0 & 0.0 & 0.5 & 1.5 & 2.5 & 4.0 & & 12.1 & 11.6 & \\
\hline 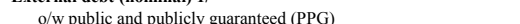 & 00 & 0.0 & 00 & & & 0.0 & 0.0 & 0.5 & 1.5 & 25 & 40 & & 12.1 & 116 & \\
\hline Change in external debt & 0.0 & 0.0 & 0.0 & & & 0.0 & 0.0 & 0.5 & 1.0 & 1.0 & 1.4 & & $\begin{array}{l}21.1 \\
0.9\end{array}$ & -1.6 & \\
\hline Identified net debt-creating flows & & & & & & & -47.4 & -43.0 & -32.6 & -35.3 & -33.9 & & -19.2 & 22.6 & \\
\hline Non-interest current account deficit & -69.7 & -71.0 & -56.8 & -29.4 & 36.4 & -52.7 & -46.2 & 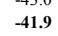 & $\begin{array}{l}-3.2 .0 \\
-31.5\end{array}$ & $\begin{array}{l}-34.3 \\
-34.3\end{array}$ & $\begin{array}{l}-33.0 \\
-33.0\end{array}$ & & -15.2 & 32.3 & 5.4 \\
\hline Deficit in balance of goods and services & -52.1 & -58.2 & -40.0 & & & -40.0 & -34.9 & -31.5 & -23.4 & -29.2 & -28.1 & & -11.6 & 35.0 & \\
\hline Exports & 81.8 & 86.2 & 79.0 & & & 78.9 & 78.6 & 77.1 & 72.1 & 71.5 & 68.7 & & 56.4 & 20.2 & \\
\hline Imports & 29.7 & 28.0 & 39.0 & & & 38.9 & 43.7 & 45.6 & 48.7 & 42.3 & 40.7 & & 44.8 & 55.2 & \\
\hline Net current transfers (negative $=$ inflow) & -16.5 & -12.5 & -16.6 & -50.9 & 46.4 & -11.9 & -10.5 & -9.6 & -7.4 & -4.4 & -4.3 & & -2.6 & -1.0 & -1.8 \\
\hline $\mathrm{o} / \mathrm{w}$ official & -16.6 & -12.4 & -15.1 & & & -11.9 & -10.5 & -9.6 & -7.4 & -4.4 & -4.3 & & -2.6 & -1.0 & \\
\hline Other current account flows (negative $=$ net inflow) & -1.1 & -0.3 & -0.2 & & & -0.8 & -0.8 & -0.8 & -0.7 & -0.6 & -0.7 & & -1.0 & -1.6 & \\
\hline Net FDI (negative = inflow) & -0.5 & -1.4 & -1.3 & -2.9 & 2.9 & -1.3 & -1.2 & -1.1 & -1.1 & -1.0 & -1.0 & & -3.6 & -9.6 & -5.7 \\
\hline Endogenous debt dynamics $2 /$ & ... & $\ldots$ & ... & & & $\ldots$ & 0.0 & 0.0 & 0.1 & -0.1 & 0.1 & & -0.3 & -0.1 & \\
\hline Contribution from nominal interest rate & & & & & & & 0.0 & 0.0 & 0.0 & 0.0 & 0.1 & & 0.3 & 0.4 & \\
\hline Contribution from real GDP growth & 0.0 & 0.0 & 0.0 & & & 0.0 & 0.0 & 0.0 & 0.0 & -0.1 & 0.0 & & -0.5 & -0.5 & \\
\hline Contribution from price and exchange rate changes & 0.0 & 0.0 & 0.0 & & & $\ldots$ & & & & & & & & & \\
\hline $\begin{array}{l}\text { Residual (3-4) } 3 / \\
\text { o/w exceptional financing }\end{array}$ & $\ldots$ & $\ldots$ & $\begin{array}{l}\ldots . \\
. . \\
\text {. }\end{array}$ & & & $\ldots$ & $\begin{array}{r}47.4 \\
0.0\end{array}$ & $\begin{array}{r}43.6 \\
0.0\end{array}$ & $\begin{array}{r}33.6 \\
0.0\end{array}$ & $\begin{array}{r}36.4 \\
0.0\end{array} \quad-10$ & $\begin{array}{r}35.3 \\
0.0\end{array}$ & & $\begin{array}{r}20.0 \\
0.0\end{array}$ & $\begin{array}{r}-24.2 \\
0.0\end{array}$ & \\
\hline PV of external debt $4 /$ & $\ldots$ & $\ldots$ & 0.0 & & & 0.0 & 0.0 & 0.4 & 1.1 & 2.0 & 3.1 & & 10.3 & 10.4 & \\
\hline In percent of exports & $\ldots$ & $\ldots$ & 0.0 & & & 0.0 & 0.0 & 0.5 & 1.6 & 2.7 & 4.5 & & 18.3 & 51.5 & \\
\hline PV of PPG external debt & $\ldots$ & $\ldots$ & 0.0 & & & 0.0 & 0.0 & 0.4 & 1.1 & 2.0 & 3.1 & & 10.3 & 10.4 & \\
\hline In percent of exports & .... & ... & 0.0 & & & 0.0 & 0.0 & 0.5 & 1.6 & 2.7 & $\begin{array}{l}3.1 \\
4.5\end{array}$ & & $\begin{array}{l}10.3 \\
18.3\end{array}$ & $\begin{array}{l}10.4 \\
51.5\end{array}$ & \\
\hline In percent of government revenues & ... & $\ldots .$. & 0.0 & & & 0.0 & 0.0 & 1.5 & $\begin{array}{l}1.0 \\
4.1\end{array}$ & 7.8 & $\begin{array}{l}4.9 \\
12.6\end{array}$ & & $\begin{array}{l}18.3 \\
47.5\end{array}$ & 55.6 & \\
\hline Debt service-to-exports ratio (in percent) & 0.0 & 0.0 & 0.0 & & & 0.0 & 0.0 & 0.0 & 0.0 & 0.0 & 0.1 & & 0.6 & 4.5 & \\
\hline $\begin{array}{l}\text { PPG debt service-to-exports ratio (in percent) } \\
\text { PPG }\end{array}$ & 0.0 & 0.0 & 0.0 & & & 0.0 & 0.0 & 0.0 & 0.0 & 0.0 & 0.1 & & 0.6 & 4.5 & \\
\hline PPG debt service-to-revenue ratio (in percent) & 0.0 & 0.0 & 0.0 & & & 0.0 & 0.0 & 0.0 & 0.0 & 0.1 & 0.2 & & 1.5 & 4.9 & \\
\hline Total gross financing need (Billions of U.S. dollars) & -1.2 & -2.1 & -1.4 & & & -1.5 & -1.4 & -1.4 & -1.0 & -1.2 & -1.2 & & -0.8 & 1.5 & \\
\hline Non-interest current account deficit that stabilizes debt ratio & -69.7 & -71.0 & -56.8 & & & -52.7 & -46.2 & -42.5 & -32.5 & -35.3 & -34.4 & & -16.1 & 33.9 & \\
\hline \multicolumn{16}{|l|}{ Key macroeconomic assumptions } \\
\hline Real GDP growth (in percent) & 57.4 & 51.0 & -24.0 & 27.0 & 29.8 & 5.8 & 6.1 & 1.9 & -8.7 & 8.2 & -1.7 & 2.0 & 5.4 & 4.1 & -0.3 \\
\hline GDP deflator in US dollar terms (change in percent) & 10.4 & 11.8 & 10.8 & 5.4 & 5.0 & 6.4 & 5.2 & 4.8 & 5.1 & 5.0 & 5.1 & 5.3 & 4.7 & 4.2 & 4.2 \\
\hline Effective interest rate (percent) 5/ & & & & & & & 1.2 & 1.2 & 2.2 & 2.4 & 2.5 & 1.9 & 2.8 & 2.9 & 2.8 \\
\hline Growth of exports of G\&S (US dollar terms, in percent) & 103.1 & 77.9 & -22.8 & 58.6 & 63.1 & 12.4 & 11.2 & 4.8 & -10.2 & 12.8 & -0.7 & 5.0 & 10.0 & 3.1 & -3.6 \\
\hline Growth of imports of G\&S (US dollar terms, in percent) & 50.6 & 59.5 & 17.3 & 10.9 & 31.0 & 12.2 & 25.3 & 11.7 & 2.5 & -1.3 & -0.6 & 8.3 & 5.8 & 6.4 & 5.8 \\
\hline Grant element of new public sector borrowing (in percent) & & & & ... & $\ldots$ & 0.0 & 36.8 & 27.7 & 23.0 & 21.0 & 20.2 & 21.5 & 12.7 & 0.0 & 8.2 \\
\hline Government revenues (excluding grants, in percent of GDP) & 20.7 & 16.3 & 20.8 & & & 22.0 & 27.6 & 26.6 & 28.3 & 25.2 & 24.8 & & 21.7 & 18.7 & 22.1 \\
\hline Aid flows (in Billions of US dollars) 7/ & 0.0 & 0.0 & 0.0 & & & 0.0 & 0.0 & 0.0 & 0.0 & 0.0 & 0.0 & & 0.0 & 0.0 & \\
\hline o/w Grants & 0.0 & 0.0 & 0.0 & & & 0.0 & 0.0 & 0.0 & 0.0 & 0.0 & 0.0 & & 0.0 & 0.0 & \\
\hline $\mathrm{o} / \mathrm{w}$ Concessional loans & 0.0 & 0.0 & 0.0 & & & 0.0 & 0.0 & 0.0 & 0.0 & 0.0 & 0.0 & & 0.0 & 0.0 & \\
\hline Grant-equivalent financing (in percent of GDP) $8 /$ & ... & $\ldots$ & $\ldots$ & & & 0.0 & 0.0 & 0.1 & 0.2 & 0.3 & 0.3 & & 0.3 & 0.0 & 0.1 \\
\hline Grant-equivalent financing (in percent of external financing) $8 /$ & .... & $\ldots$ & $\ldots$ & & & 100.0 & 36.8 & 27.7 & 23.0 & 21.0 & 20.2 & & 12.7 & $\ldots$ & 9.5 \\
\hline \multicolumn{16}{|l|}{ Memorandum items: } \\
\hline Nominal GDP (Billions of US dollars) & 1.7 & 2.9 & 2.4 & & & 2.7 & 3.0 & 3.2 & 3.1 & 3.5 & 3.6 & & 4.4 & 6.2 & \\
\hline Nominal dollar GDP growth & 73.7 & 68.8 & -15.8 & & & 12.6 & 11.6 & 6.8 & -4.0 & 13.7 & 3.3 & 7.3 & 10.3 & 8.5 & 3.8 \\
\hline PV of PPG external debt (in Billions of US dollars) & & & 0.0 & & & 0.0 & 0.0 & 0.0 & 0.0 & 0.1 & 0.1 & & 0.5 & 0.6 & \\
\hline (PVt-PVt-1)/GDPt-1 (in percent) & & & & & & 0.0 & 0.0 & 0.4 & 0.7 & 1.1 & 1.3 & 0.6 & 1.9 & -0.5 & 0.9 \\
\hline Gross workers' remittances (Billions of US dollars) & 0.0 & 0.0 & 0.0 & & & 0.0 & 0.0 & 0.0 & 0.0 & 0.0 & 0.0 & & 0.0 & 0.0 & \\
\hline PV of PPG external debt (in percent of GDP + remittances) & $\ldots$ & & 0.0 & & & 0.0 & 0.0 & 0.4 & 1.1 & 2.0 & 3.1 & & 10.3 & 10.4 & \\
\hline PV of PPG external debt (in percent of exports + remittances) & $\ldots$ & $\ldots$ & 0.0 & & & 0.0 & 0.0 & 0.5 & 1.6 & 2.7 & 4.5 & & 18.3 & 51.5 & \\
\hline Debt service of PPG external debt (in percent of exports + remittances) & $\ldots$ & $\ldots$ & 0.0 & & & 0.0 & 0.0 & 0.0 & 0.0 & 0.0 & 0.1 & & 0.6 & 4.5 & \\
\hline
\end{tabular}

Debt service of PPG external debt (in percent of exports + remittances)

Sources: Country authorities; and staff estimates and projections.

$1 /$ Includes only public sector external debt.

Derived as $[\mathrm{r}-\mathrm{g}-\rho(1+\mathrm{g})](1+\mathrm{g}+\rho+\mathrm{g} \rho)$ times previous period debt ratio, with $\mathrm{r}=$ nominal interest rate; $\mathrm{g}=$ real GDP growth rate, and $\rho=$ growth rate of GDP deflator in U.S. dollar term

Assumes that PV of private sector debt is equivalent to its fice

5/ Current-year interest payments divided by previous period debt stock.

6/ Historical averages and standard deviations are generally derived over the past 10 years, subject to data availability. Changing all historical averages to a horizon of 8 years does not affect the magnitude of the shocks and, therefore, the outcome of the 7/

grants, concessional loans, and debt relief.

8/ Grant-equivalent financing includes grants provided directly to the government and through new borrowing (difference between the face value and the $\mathrm{PV}$ of new debt). 
Table 2.Timor-Leste: Sensitivity Analysis for Key Indicators of Public and Publicly Guaranteed External Debt, 2010-2030 (In percent)

\begin{tabular}{l}
\hline \\
\hline Baseline \\
A. Alternative Scenarios \\
A1. Key variables at their historical averages in 2010-2030 1/ \\
A2. New public sector loans on less favorable terms in 2010-2030 2 \\
B. Bound Tests \\
B1. Real GDP growth at historical average minus one standard deviation in 2011-2012 \\
B2. Export value growth at historical average minus one standard deviation in 2011-2012 3/ \\
B3. US dollar GDP deflator at historical average minus one standard deviation in 2011-2012 \\
B4. Net non-debt creating flows at historical average minus one standard deviation in 2011-2012 4/ \\
B5. Combination of B1-B4 using one-half standard deviation shocks \\
B6. Customized export shock
\end{tabular}

PV of debt-to-exports ratio

Baseline

A. Alternative Scenarios

A1. Key variables at their historical averages in 2010-2030 1/

A2. New public sector loans on less favorable terms in 2010-2030 2

B. Bound Tests

B1. Real GDP growth at historical average minus one standard deviation in 2011-2012

B2. Export value growth at historical average minus one standard deviation in 2011-2012 3 /

B3. US dollar GDP deflator at historical average minus one standard deviation in 2011-2012

B4. Net non-debt creating flows at historical average minus one standard deviation in 2011-2012 4/

B5. Combination of B1-B4 using one-half standard deviation shocks

B6. Customized export shock

PV of debt-to-revenue ratio

Baseline

A. Alternative Scenarios

A1. Key variables at their historical averages in 2010-2030 1/

A2. New public sector loans on less favorable terms in 2010-2030 2

Projections

$2010 \quad 2011-2012-2013-2014$

0

0

2

10

10

0

0

$\begin{array}{rrrrrrr}0 & 0 & 1 & 2 & 4 & \mathbf{1 2} & 12 \\ 10 & 25 & 28 & 26 & 27 & \mathbf{3 2} & 22 \\ 0 & 0 & 1 & 2 & 3 & \mathbf{1 1} & 11 \\ 6 & 12 & 14 & 14 & 15 & \mathbf{2 1} & 16 \\ -25 & -63 & -68 & -61 & -60 & \mathbf{- 4 6} & -22 \\ 4 & 8 & 9 & 9 & 11 & \mathbf{1 7} & 14\end{array}$

\section{B. Bound Tests}

B1. Real GDP growth at historical average minus one standard deviation in 2011-2012

B2. Export value growth at historical average minus one standard deviation in 2011-2012 3/

B3. US dollar GDP deflator at historical average minus one standard deviation in 2011-2012

B4. Net non-debt creating flows at historical average minus one standard deviation in 2011-2012 4/

B5. Combination of B1-B4 using one-half standard deviation shocks

B6. Customized export shock 


\section{Debt service-to-exports ratio}

\section{Baseline}

\section{A. Alternative Scenarios}

A1. Key variables at their historical averages in 2010-2030 1/

A2. New public sector loans on less favorable terms in 2010-2030 2

\section{B. Bound Tests}

B1. Real GDP growth at historical average minus one standard deviation in 2011-2012

B2. Export value growth at historical average minus one standard deviation in 2011-2012 3

B3. US dollar GDP deflator at historical average minus one standard deviation in 2011-2012

B4. Net non-debt creating flows at historical average minus one standard deviation in 2011-2012

B5. Combination of B1-B4 using one-half standard deviation shocks

B6. Customized export shock

\section{Debt service-to-revenue ratio}

\section{Baseline}

A. Alternative Scenarios

A1. Key variables at their historical averages in 2010-2030 1/

A2. New public sector loans on less favorable terms in 2010-2030 2

0

\section{B. Bound Tests}

B1. Real GDP growth at historical average minus one standard deviation in 2011-2012

B2. Export value growth at historical average minus one standard deviation in 2011-2012 3 /

B3. US dollar GDP deflator at historical average minus one standard deviation in 2011-2012

B4. Net non-debt creating flows at historical average minus one standard deviation in 2011-2012 4

B Combination of B1-B4 using one-half standard deviation shocks

B6. Customized export shock

Memorandum item:

Grant element assumed on residual financing (i.e., financing required above baseline) 5

0

$\begin{array}{rrrrrrr}0 & 0 & 0 & 0 & 0 & \mathbf{1} & 5 \\ 0 & 1 & 2 & 1 & 2 & \mathbf{5} & 14 \\ 0 & 0 & 0 & 0 & 0 & \mathbf{1} & 5 \\ 0 & 0 & 1 & 1 & 1 & \mathbf{2} & 8 \\ 0 & -1 & -3 & -2 & -2 & \mathbf{- 6} & -11 \\ 0 & 0 & 0 & 0 & 0 & \mathbf{2} & 7\end{array}$

Sources: Country authorities; and staff estimates and projections.

1/ Variables include real GDP growth, growth of GDP deflator (in U.S. dollar terms), non-interest current account in percent of GDP, and non-debt creating flows.

2/ Assumes that the interest rate on new borrowing is by 2 percentage points higher than in the baseline., while grace and maturity periods are the same as in the baseline.

3/ Exports values are assumed to remain permanently at the lower level, but the current account as a share of GDP is assumed to return to its baseline level after the shock (implicitly assuming an offsetting adjustment in import levels).

$4 /$ Includes official and private transfers and FDI.

5/ Applies to all stress scenarios except for A2 (less favorable financing) in which the terms on all new financing are as specified in footnote 2. 
Table 3.Timor-Leste: Public Sector Debt Sustainability Framework, Baseline Scenario, 2007-2030

(In percent of GDP, unless otherwise indicated)

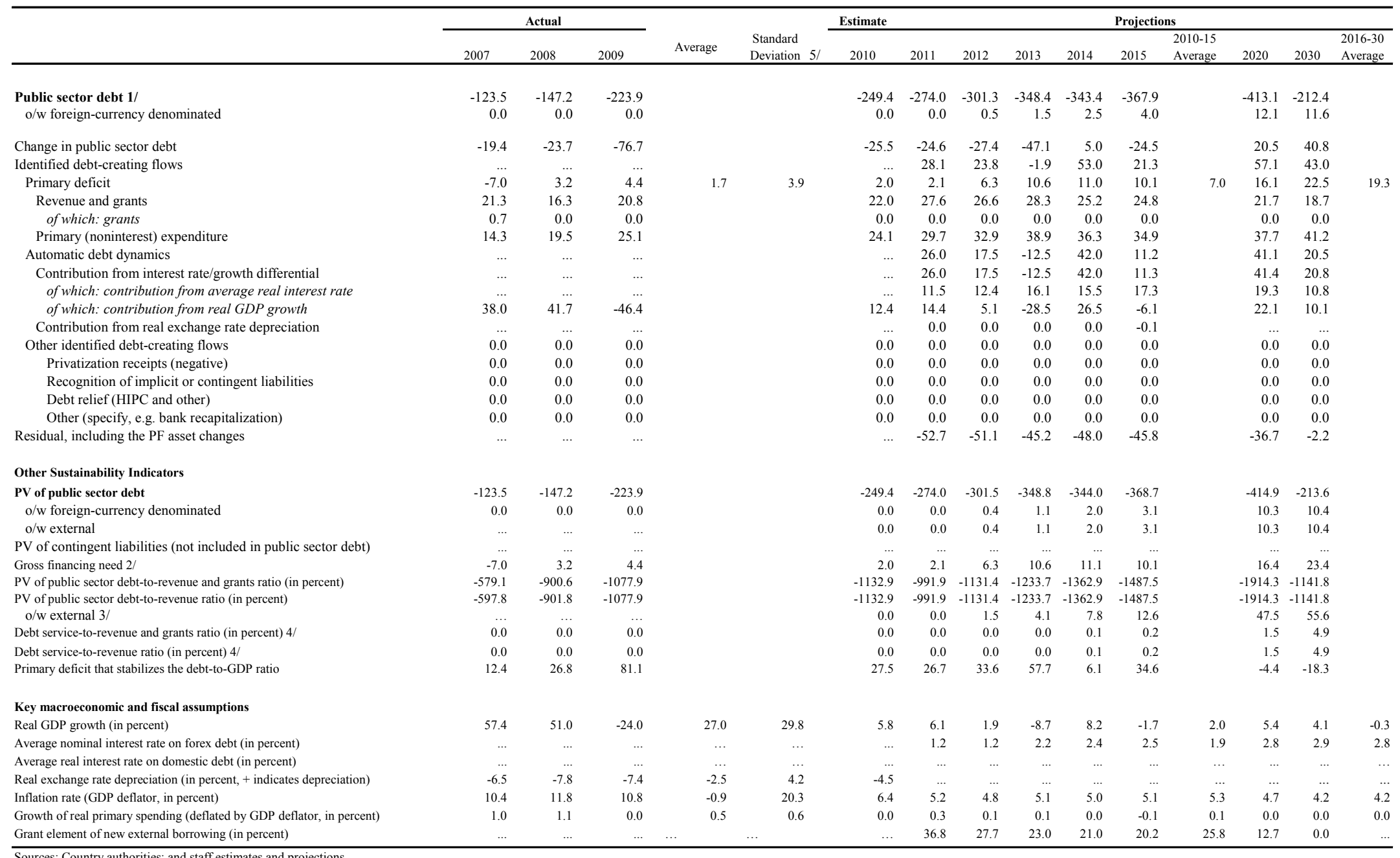

Sources: Country authorities; and staff estimates and projections.

1/ Based on net central government debt (i.e., gross debt minus the PF assets).

2/ Gross financing need is defined as the primary deficit plus debt service plus the stock of short-term debt at the end of the last period.

$3 /$ Revenues excluding grants.

$4 /$ Debt service is defined as the sum of interest and amortization of medium and long-term debt.

$5 /$ Historical averages and standard deviations are generally derived over the past 10 years, subject to data availability. Changing all historical averages to a horizon of 8 years does not affect the magnitude of the shocks and, therefore, the outcome of the analysis. 


\begin{tabular}{|c|c|c|c|c|c|c|c|c|}
\hline & \multicolumn{8}{|c|}{ Projections } \\
\hline & 2010 & 2011 & 2012 & 2013 & 2014 & 2015 & 2020 & 2030 \\
\hline \multicolumn{9}{|c|}{ PV of Debt-to-GDP Ratio } \\
\hline Baseline & -249 & -274 & -301 & -349 & -344 & -369 & -415 & -214 \\
\hline \multicolumn{9}{|l|}{ A. Alternative scenarios } \\
\hline A1. Real GDP growth and primary balance are at historical averages & -249 & -229 & -204 & -173 & -148 & -123 & -42 & 3 \\
\hline A2. Primary balance is unchanged from 2010 & -249 & -274 & -305 & -360 & -363 & -395 & -493 & -456 \\
\hline A3. Permanently lower GDP growth $1 /$ & -249 & -276 & -307 & -358 & -356 & -384 & -448 & -222 \\
\hline \multicolumn{9}{|l|}{ B. Bound tests } \\
\hline B1. Real GDP growth is at historical average minus one standard deviations in 2011-2012 & -249 & -297 & -339 & -389 & -381 & -407 & -447 & -195 \\
\hline B2. Primary balance is at historical average minus one standard deviations in 2011-2012 & -249 & -271 & -299 & -346 & -342 & -367 & -414 & -216 \\
\hline B3. 10 percent of GDP increase in other debt-creating flows in 2011 & -249 & -265 & -293 & -340 & -336 & -360 & -408 & -210 \\
\hline \multicolumn{9}{|c|}{ PV of Debt-to-Revenue Ratio 2/ } \\
\hline Baseline & -1133 & -992 & -1131 & -1234 & -1363 & -1488 & -1914 & -1142 \\
\hline \multicolumn{9}{|l|}{ A. Alternative scenarios } \\
\hline A1. Real GDP growth and primary balance are at historical averages & -1133 & -829 & -767 & -612 & -585 & -498 & -193 & 15 \\
\hline A2. Primary balance is unchanged from 2010 & -1133 & -992 & -1146 & -1275 & -1437 & -1592 & -2277 & -2436 \\
\hline A3. Permanently lower GDP growth $1 /$ & -1133 & -1001 & -1151 & -1266 & -1409 & -1550 & -2066 & -1189 \\
\hline \multicolumn{9}{|l|}{ B. Bound tests } \\
\hline B1. Real GDP growth is at historical average minus one standard deviations in 2011-2012 & -1133 & -1075 & -1273 & -1377 & -1511 & -1640 & -2060 & -1043 \\
\hline B2. Primary balance is at historical average minus one standard deviations in 2011-2012 & -1133 & -981 & -1123 & -1225 & -1354 & -1479 & -1909 & -1153 \\
\hline B3. 10 percent of GDP increase in other debt-creating flows in 2011 & -1133 & -960 & -1099 & -1201 & -1330 & -1454 & -1881 & -1122 \\
\hline
\end{tabular}

Debt Service-to-Revenue Ratio 2/

Baseline

\section{A. Alternative scenarios}

A1. Real GDP growth and primary balance are at historical averages

A2. Primary balance is unchanged from 2010

A3. Permanently lower GDP growth $1 /$

$\begin{array}{rrrrrrrr}0 & 0 & 0 & 0 & 0 & -1 & 0 & 1 \\ 0 & 0 & 0 & 0 & -1 & -2 & -9 & -56 \\ 0 & 0 & 0 & 0 & 0 & 1 & 3 & 17\end{array}$

\section{B. Bound tests}

B1. Real GDP growth is at historical average minus one standard deviations in 2011-2012

B2. Primary balance is at historical average minus one standard deviations in 2011-2012

B3. 10 percent of GDP increase in other debt-creating flows in 2011

$\begin{array}{rrrrrrrr}0 & 0 & 0 & 1 & 1 & 2 & 7 & 22 \\ 0 & 0 & 0 & 0 & 0 & 1 & 2 & 6 \\ 0 & 0 & 1 & 1 & 1 & 1 & 4 & 8\end{array}$

Sources: Country authorities; and staff estimates and projections.

1/ Assumes that real GDP growth is one percentage point below the baseline.

2/ Revenues are defined inclusive of grants. 
Statement by the IMF Staff Representative on the Democratic Republic of Timor-Leste January 19, 2011

1. This statement provides information that has become available since the Staff Report was circulated to the Executive Board on December 3, 2010. The information does not alter the thrust of the staff appraisal.

2. Timor-Leste's economic growth picked up in the second half of 2010. Preliminary data provided by the authorities indicate that GDP growth in 2010 is likely to be higher than the estimate in the Staff Report of 6 percent and closer to the government's estimate of 91/2 percent:

- $\quad$ Bad weather conditions in 2010 appear to have had a more limited impact on agricultural output than expected. Preliminary estimates indicate that, despite unseasonally high rainfalls, total agricultural production grew by $1 / 2$ percent in 2010 , rather than declined by 5 percent as estimated by staff.

- Preliminary government estimates of activity in industry and services also show strongerthan-expected growth of 16 percent in 2010. Activity was particularly strong in the transport and communication, financial services, and the small manufacturing sectors.

3. Government revenue from petroleum was higher than budgeted, because of higher-than-projected oil prices: US $\$ 2.2$ billion was collected in petroleum revenue for 2010, 30 percent above the mid-year 2010 budget estimate. If higher oil prices persist, sustainable income (ESI) from petroleum could be revised up by 3 to 5 percent when the annual recalculation is made in April.

4. The Ministry of Finance has revised upwards its estimate of government cash spending in 2010 to US\$729 million, about 20 percent higher than in 2009. Spending remained below the sum of expected 2010 domestic revenue and the estimated sustainable income (ESI) from petroleum by about US $\$ 100$ million.

5. The Banking and Payments Authority (BPA) reported an increase in CPI inflation for Dili to 9.1 percent year-on-year in November 2010. The rate increased from the 7.5 percent reported in the Staff Report for September.

6. As noted in the staff appraisal, a higher ESI creates room for additional spending. Staff supports government investment in public infrastructure, health and education, and their efforts to reduce administrative and absorptive capacity constraints. Meanwhile, spending at a more measured pace would stand a better chance of realizing high quality investment, crowding in private investment, and thereby achieving balanced and sustainable growth. The higher-thanexpected inflation also underlines the need for fiscal restraint. 


\section{INTERNATIONAL MONETARY FUND}

Public Information Notice (PIN) No. 11/31

FOR IMMEDIATE RELEASE

March 8, 2011
International Monetary Fund

$70019^{\text {th }}$ Street, NW

Washington, D. C. 20431 USA

\section{IMF Executive Board Concludes 2010 Article IV Consultation with the Democratic Republic of Timor-Leste}

On January 28, 2011, the Executive Board of the International Monetary Fund (IMF) concluded the Article IV consultation ${ }^{1}$ with Timor-Leste.

\section{Background}

The Timorese economy has grown fast over the past three years in an environment of improved security following the 2006 civil unrest. Driven by higher oil-financed public spending and a rebound in agriculture from the 2007 drought, non-oil growth averaged 11 percent during 2007-09. A recent estimate by the World Bank also shows a decline of poverty incidence from 50 percent in 2007 to 41 percent in 2009 . With increased public spending, domestic price pressures have recently been rising and are particularly high in some segments of the economy, such as housing and construction.

The fiscal position strengthened in 2009, the non-oil fiscal deficit declined by 17 percentage points to 92 percent of non-oil GDP. This reflected a moderation of

\footnotetext{
${ }^{1}$ Under Article IV of the IMF's Articles of Agreement, the IMF holds bilateral discussions with members, usually every year. A staff team visits the country, collects economic and financial information, and discusses with officials the country's economic developments and policies. On return to headquarters, the staff prepares a report, which forms the basis for discussion by the Executive Board. At the conclusion of the discussion, the Managing Director, as Chairman of the Board, summarizes the views of Executive Directors, and this summary is transmitted to the country's authorities. An explanation of any qualifiers used in summings up can be found here: http://www.imf.org/external/np/sec/misc/qualifiers.htm.
} 
spending growth to 8 percent following a sharp increase of 130 percent in 2008 . A supplemental 2010 budget raised the spending envelop by more than 30 percent to US\$838 million, to be financed by withdrawals of US\$811 million from the Petroleum Fund (PF). However, due to procurement delays actual public spending is expected to be around US $\$ 729$ million, and the non-oil fiscal deficit is projected at about 100 percent of non-oil GDP.

Timor-Leste stands out as the most oil-dependent economy in the world. In 2009, petroleum income accounted for about 95 percent of total government revenue and almost 80 percent of gross national income (GNI). The PF assets reached US\$6.8 billion or about ten times non-oil GDP in October 2010. The current account surplus has been driven by large oil income.

The financial sector remains at an embryonic stage of development. Three foreign bank branches dominate the financial sector, in addition to a small state-owned microfinance institution. A first insurance company has been established. Despite high bank deposit growth, private sector credit has remained stagnant, hampered by a large share of nonperforming loans, weak contract enforcement, and unclear property rights.

The near-term outlook remains positive, supported by social and political stability and government spending. Preliminary data provided by the authorities indicate that GDP growth in 2010 is likely to be higher than the estimate in the Staff Report of 6 percent and closer to the government's estimate of $9 \frac{1}{2}$ percent, potentially pointing to a higher growth projection in 2011. Staff expects GDP growth to stay high in the medium term. Inflation is projected to increase from $4 \frac{1}{2}$ percent to 6 percent in 2011 , higher than the average of neighboring countries. Large uncertainties surround the medium-term outlook, including the path of oil prices and production, the magnitude and quality of public spending, and progress in business-enabling structural reforms.

\section{Executive Board Assessment}

Executive Directors welcomed Timor-Leste's economic performance over the last three years and the progress made on poverty reduction and other social indicators. Following a slowdown caused by adverse weather, the medium-term outlook for growth is positive. Going forward, Timor-Leste's key challenge remains to use its petroleum wealth wisely to build a strong non-oil economy and raise living standards.

Directors welcomed the government's resolve to push ahead with its development policies. They noted that carefully selected infrastructure investment could have sufficiently high returns to justify temporary withdrawals from the PF in excess of the estimated sustainable income (ESI), and that the upward revision in the ESI has created room for additional capital expenditure. Most Directors observed, however, that spending at a slower-than-planned pace, consistent with the absorptive capacity of the economy, would stand a better chance of realizing high quality projects and crowding in private investment. 
Directors praised the government's emphasis on raising the country's ability to plan, evaluate, and implement spending programs, so that any expansion of public works does not overwhelm administrative capacity. They supported plans to create a new agency, reporting directly to the Prime Minister, which will be in charge of major project evaluation and approval. Further improvements in public financial management and budget execution will also be important.

Directors welcomed the government's efforts to make the regulatory environment more business-friendly. They noted that the introduction of a one-stop-shop for start-ups is a step in this direction. Accelerated reforms to enhance property rights, such as the pending land law, would also make it easier for the financial sector to extend credits to local businesses.

Directors observed that the official dollarization has helped keep inflation under control and should be maintained, supported by a fiscal policy that is mindful of external stability. Productivity-enhancing structural reforms and efforts to build labor skills would improve competitiveness in non-oil industries and services. Directors also noted that a slowerthan-envisaged pace of public spending growth would help contain inflation pressures as supply-side limitations are approached.

Directors called for an intensification of the engagement between Fund staff and the authorities. Noting differences between the official and the more conservative staff's growth projections, they encouraged a closer policy dialog and greater use of technical assistance, including to strengthen Timor-Leste's economic statistics. A number of Directors also saw merit in reopening the resident representative office.

Public Information Notices (PINs) form part of the IMF's efforts to promote transparency of the IMF's views and analysis of economic developments and policies. With the consent of the country (or countries) concerned, PINs are issued after Executive Board discussions of Article IV consultations with member countries, of its surveillance of developments at the regional level, of post-program monitoring, and of ex post assessments of member countries with longer-term program engagements. PINs are also issued after Executive Board discussions of general policy matters, unless otherwise decided by the Executive Board in a particular case. The staff report (use the free Adobe Acrobat Reader to view this pdf file) for the 2010 Article IV Consultation with the Democratic Republic of TimorLeste is also available. 
Democratic Republic of Timor-Leste: Selected Economic Indicators, 2005-11

\begin{tabular}{|c|c|c|c|c|c|c|c|}
\hline & 2005 & 2006 & 2007 & 2008 & $\begin{array}{c}2009 \\
\text { Prel. }\end{array}$ & $\begin{array}{c}2010 \\
\text { Proj. }\end{array}$ & $\begin{array}{r}2011 \\
\text { Proj. }\end{array}$ \\
\hline \multicolumn{8}{|l|}{ Output and prices } \\
\hline Non-oil GDP at current prices (US\$ million) & 332 & 327 & 358 & 444 & 556 & 627 & 708 \\
\hline Real non-oil GDP growth (percentage change) & 6.2 & -5.8 & 9.1 & 11.0 & 12.9 & 6.1 & 7.3 \\
\hline Including United Nations $1 /$ & 2.3 & -3.4 & 18.2 & 10.6 & 9.1 & 5.0 & 6.2 \\
\hline \multirow[t]{2}{*}{ Inflation (CPI, percentage change, period average) } & 1.8 & 4.1 & 8.9 & 7.6 & 0.1 & 4.5 & 6.0 \\
\hline & \multicolumn{7}{|c|}{ (In percent of non-oil GDP) } \\
\hline \multicolumn{8}{|l|}{ Investment-saving balance } \\
\hline Gross investment 2/ & 21 & 23 & 29 & 63 & 55 & 48 & 70 \\
\hline Gross national savings & 99 & 188 & 358 & 518 & 301 & 276 & 267 \\
\hline Domestic revenues & 11 & 10 & 12 & 10 & 11 & 11 & 11 \\
\hline Petroleum revenue & 107 & 195 & 367 & 540 & 332 & 328 & 323 \\
\hline Grants & 10 & 0 & 3 & 0 & 0 & 0 & 0 \\
\hline Expenditure (cash basis) & 26 & 32 & 66 & 120 & 103 & 100 & 123 \\
\hline Recurrent expenditure & 19 & 27 & 51 & 81 & 65 & 72 & 73 \\
\hline Capital expenditure & 7 & 5 & 15 & 39 & 38 & 28 & 50 \\
\hline Overall balance & 102 & 174 & 316 & 431 & 239 & 239 & 210 \\
\hline Non-oil fiscal balance & -5 & -21 & -51 & -110 & -92 & -89 & -113 \\
\hline \multicolumn{8}{|l|}{ Combined sources fiscal operations $3 /$} \\
\hline \multirow[t]{2}{*}{ Net domestic assets (end-period) } & -24 & -3 & -48 & -42 & -30 & $\ldots$ & $\ldots$ \\
\hline & \multicolumn{7}{|c|}{ (In millions of U.S. dollars) } \\
\hline \multicolumn{8}{|l|}{ External sector } \\
\hline Current account & 261 & 541 & 1,177 & 2,023 & 1,363 & 1,425 & 1,395 \\
\hline Merchandise exports 2/ & 8 & 9 & 7 & 14 & 9 & 11 & 14 \\
\hline \multirow[t]{2}{*}{ Merchandise imports } & 112 & 101 & 176 & 311 & 385 & 575 & 802 \\
\hline & \multicolumn{7}{|c|}{ (In percent of non-oil GDP) } \\
\hline Current account & 79 & 166 & 329 & 456 & 245 & 227 & 197 \\
\hline Merchandise exports 2/ & 2 & 3 & 2 & 3 & 2 & 2 & 2 \\
\hline Merchandise imports & 34 & 31 & 49 & 70 & 69 & 92 & 113 \\
\hline \multicolumn{8}{|l|}{ Memorandum item: } \\
\hline Petroleum Fund balance (percent of non-oil GDP) 5/ & 112 & 310 & 583 & 945 & 968 & 1,076 & 1,169 \\
\hline
\end{tabular}

Sources: Data provided by the Timor-Leste authorities; and IMF staff estimates.

1 / Includes locally paid compensation of UN peacekeeping mission staff.

2/ Excludes oil/gas sector.

$3 /$ Includes autonomous agencies and quasi-fiscal expenditure by donors outside the central government budget. The revenue decline in 2005

reflects the creation of the Petroleum Fund to which all oil revenue now accrues. Income from the fund and donor assistance finances the deficit.

4/ Excludes currency holdings by the public, for which no data are available.

5/ End-period. Figure for 2004 refers to the Timor-Sea account, which preceded the August 2005 establishment of the Petroleum Fund. 


\section{Statement by Mr. Arrigo Sadun, Executive Director for the \\ Democratic Republic of Timor-Leste \\ and Mr. José Cardoso, Advisor to the Executive Director \\ January 19, 2011}

We thank staff for visiting Timor-Leste. The authorities of Timor-Leste have always highly valuated the dialogue with the IMF, not only under the framework of Article IV consultations, but also in the context of important technical assistance missions.

The authorities of Timor-Leste do not consider the 2010 Article IV staff report to reflect appropriately the most important recent economic and social developments, and also the medium- and long-run prospects of Timor-Leste. The authorities would have preferred a more appropriate guidance of the mission, and consider that the insufficient understanding of Timor-Leste's reality has undermined the quality of the report. Additionally, this is the first Article IV consultation after the closing of the IMF Resident Representative (ResRep) Office in June 2009. In this regard, in the Buff statement for the last Article IV staff report, we had already expressed that "the authorities expect, under the new circumstances, a deeper engagement of the Fund given the extremely challenging endeavors that Timor-Leste faces ahead".

At the last Article IV discussion - and also at the IMF/WB Spring and Annual Meetings, and other bilateral contacts - the authorities have regretted the Fund's decision of closing the ResRep Office. In a fragile and post-conflict country, with less than a decade of existence, the presence of a ResRep Office makes a difference for Timor-Leste, and apparently also for the IMF. We recall the fact that the decision of closing the ResRep Office was motivated, at the time, by financial constrains and in the context of the IMF downsizing. Such motivation no longer persists. Once again, the authorities of Timor-Leste would like to express their interest in the reopening of the ResRep Office in Díli.

The following paragraphs present what the Timor-Leste authorities consider the most important disagreements with the Article IV staff report:

- Front page; point 2: On growth prospects, the staff report says, "in 2010 non-oil GDP growth is expected to slow to around 6 percent due to the impact of adverse weather on harvests". The authorities' estimate of non-oil GDP growth in 2010 is higher at 9.5 percent. Food production has been affected by unseasonal rains, but the share of agriculture in the non-oil GDP is relatively small at 26 percent, and the absence of growth in agriculture has been more than compensated for by buoyancy in the sectors of transport and communications, manufacturing, wholesale and retail trade, and private construction.

- The past experience is that staff projections of non-oil GDP growth in Timor- 
Leste have been on the pessimistic side. For example, following the 2009 Article IV consultation, staff had estimated 7.4 percent for the 2009 growth rate, whereas the authorities had estimated 12.2 percent. The actual outturn for 2009 was higher than the staff's figure, and the authorities' figure has even proved to be slightly on the conservative side. The actual outturn for the 2009 growth rate was (in the 2010 Article IV staff report) 12.9 percent.

- Front page; point 2: The staff report says "there is uncertainty about the size, timing and quality of capital spending". The authorities feel that the finalization of the Strategic Development Plan (SDP) for 2011-2030, and the proposed establishment of the Infrastructure Fund in 2011 will contribute to a reduction in the uncertainties about the future path of public investment. This multi-annual and fungibility fund will allow the authorities to review the proposed projects and Bill of Quantities (BoQs) with the aim to reduce inefficiencies. The government is committed to ensuring high quality of public spending and is in no hurry to implement projects by December 31, if this hurrying can produce inefficiencies.

- Page 4, paragraph 6: The staff report says "given procurement delays, primarily for capital spending, staff suggests that 2010 cash spending will be around $\$ 650$ million, well below the revised spending envelope". Once again, this has proven to be on the pessimistic side, as the actual estimate of cash spending in 2010 is $\$ 731.9$ million, representing a rate of execution of 87.3 percent (even higher than the conservative authorities' estimation). Moreover, the budget execution rate will be, at the end, somewhat higher, because according to the prevailing international standards, closure of accounts is only fully booked two months after the end of the concerned financial year. It is important to highlight that, over the last few years, the budget execution rate has been rising steadily.

- Page 5, paragraph 9: According to the staff report, "GDP growth is expected to increase to around $7 \frac{1}{3}$ percent in 2011 ". The authorities expect close to double-digit growth in 2011 based on the recovery of agriculture, rapid buildup in capital spending, and continued buoyancy in the private sector especially in construction, trade and transport, wholesale and retail trade, financial services, and public utilities.

- Page 5, paragraph 9: The staff report says that "non-oil growth is to gradually converge to around 6 percent in the long-run". The authorities expect a higher growth rate to be sustainable in the long-run once the productive capacity of the economy has been substantially augmented by the planned investments in infrastructure and human capital development. Coupled with contemplated improvements in the business environment, this will provide for the emergence of a strong private sector, both domestic and foreign, beyond 2015. Thus, a double digit growth can be sustained in the next two decades. 
- Page 6, paragraph 11: The authorities had suggested to staff to provide upper and lower bounds for projections, namely regarding economic growth. These projections are given on page 8 in the two tables on Medium-Term Fiscal Outlook. For 2011, the range of the projected growth rate is 6.1-7.3 percent, and the average for 2012-2015 is 7.8-9.0 percent. The authorities' analysis, based on the reasons presented above, is that the upper bound is a double-digit growth rate in this period, as well as beyond 2015.

- Page 8, Table on Medium-Term Fiscal Outlook: Given the new basis for the estimated sustainable income (ESI), the staff report projects ESI at \$734 million in 2011 , rising to $\$ 740$ million by 2015 in the Baseline Scenario, and to $\$ 771$ million in the Alternative Scenario. These projections appear to be predicated on very conservative assumptions about the future outlook for oil prices. According to Table 7, crude oil prices are expected to be at $\$ 68$ per barrel in 2011, rising to $\$ 81$ by 2015 . It is important to highlight that oil prices have already approached $\$ 90$ per barrel, and that, as the world economy recovers, they could rise even further.

- Page 8, paragraph 16: The staff report says "Within current spending, there is room to restrain wage growth, phase out subsidies, including on rice and electricity and to better target transfers to the most needy". The authorities would like to indicate that a reduction is proposed in the level of public transfers in the budget for 2011, while emphasizing that the policy of social protection is important from the viewpoint of containing poverty, and preserving peace and stability, through support that targets the most vulnerable groups, such as veterans, the elderly, internally displaced persons (IDPs), widows, and invalids.

- Page 9, paragraph 17: The staff report highlights the low domestic tax to non-oil GDP ratio of only 8 percent. The authorities fully recognize the need for reforms, both in tax administration and tax policy, not only from the viewpoint of mobilizing more resources, but also for redistributive purposes. A phased strategy is being implemented with an initial focus on improvements in tax administration, taxpayer education, and measures to improve compliance, including expansion in the regime of withholding tax. Beyond this, it is proposed to broaden the tax base, enhance the tax system, and raise some tax rates, namely on luxury goods and services.

- Page 9, paragraph 20: The staff report indicates that "significant gaps remain in PFM". The authorities have placed great emphasis on improvements in public financial management (PFM) in recent years, which has led to a substantial enhancement in the budget execution rate. A Strategic Plan for PFM was finalized in early 2010 and is being implemented in key areas related to accounting systems, procurement practices, internal audit, staff capacity building, etc. Strong anticorruption mechanisms are being put in place. Two important facts should have been highlighted: (i) it is reassuring that the Transparency International Corruption 
Perceptions Index of 2010 shows an improvement of 19 positions by Timor-Leste in the past 12 months; and (ii) Timor-Leste was the third country worldwide to achieve the status of full compliance with the Extractive Industry Transparency Initiative.

- Page 10, paragraph 21: The staff report says that "ambiguity as to who will be the manager of the Petroleum Fund is likely to create uncertainty". We highlight the fact that the authorities propose to provide flexibility in the Petroleum Fund Law as to which institution would be the Operational Manager of the Petroleum Fund in the future. The Banking and Payments Authority (BPA) of Timor-Leste is currently assigned to conduct this task, but the government proposes that it may be the future Central Bank or any other public entity established by the National Parliament in the future. This entity will be independent from the government, and there will be a clear division of responsibilities between the overall manager of the Petroleum Fund (the government) and the Operational Manager.

- Page 15, Box 1: The staff report says "In the medium run, the current account surplus is projected to remain below its sustainable level by about 30 percent of the GDP". This seems to be a biased and non-clear normative approach for estimating the "sustainable" level, especially when the surplus remains high at over 100 percent of the non-oil GDP up to 2015.

- Page 25: Table on Medium-Term Outlook: A number of projections have been made in the staff report of the Medium-Term Outlook for Timor-Leste during the period, 2011-2015. The authorities have the following views on the projections:

(a) Non-oil GDP growth rate: The average growth rate projected for the next five years is 8.7 percent. However, the authorities are confident that attaining an average of a double-digit growth rate over the period is feasible in light of the fact that growth will start from a low base in a relatively small economy, and that plans, policies, and institutions will be put in place to achieve this growth rate. The growth rate will be driven by public capital spending, the increase of agricultural productivity, and continued high growth in the industry and services sector.

(b) Domestic revenue: This is shown as remaining, more or less, constant at 11 percent of GDP up to 2015. The authorities are working with the target of 20 percent of GDP in the next five years.

(c) Capital expenditure: Central government capital expenditure is projected to reach a peak of 62 percent of the GDP in 2013 and then fall sharply to 44 percent by 2015 . The authorities feel that the level of public investment as a percentage of the non-oil GDP will decline less rapidly and that it will be sustained by larger financing through domestic revenues. In addition, the better prospects for private investment due to improved infrastructure and a business- 
friendly environment will preserve the growth momentum.

(d) Crude oil price: As already mentioned, the forecast for crude oil price, as highlighted above, is conservative at $\$ 68$ per barrel in 2011 , rising to $\$ 81$ by 2015. The price is already close to $\$ 90$ per barrel and the outlook is bullish given recovery in the world economy. A higher price of oil than projected will, of course, mean a higher ESI, a larger balance in the Petroleum Fund, and a higher surplus in the current account.

Overall, the authorities appreciate the fact that the staff report for the 2010 Article IV Consultation recognizes the post-conflict strong economic recovery in this fragile state since 2006, with significant reduction in the incidence of poverty and major strides in key social indicators. However, the report is (again, proven by existing data) excessively biased on the pessimistic side regarding the strong potential for a double-digit growth rate in the medium- to long-run. The current momentum will be boosted by infrastructure and human capital development (financed mostly by oil revenues), which will also be promoted by the emergence of the nascent and dynamic private sector, especially the industry and services. As this potential gets recognition by the international markets, foreign direct investment could also flow into Timor-Leste. The transition from conflict to development that has taken place amidst the recently found peace, stability, and growth holds out the prospects that Timor-Leste will join the rank of upper middleincome countries within the next two decades, as envisioned by the SDP.

To conclude, we would like to express two very positive and encouraging assessments. First, the UNDP 2010 Human Development Report has recently been released, and it now ranks Timor-Leste at 120 out of 169 countries, based on 2009/2010 data. This represents a spectacular improvement in Timor-Leste's relative position (from 162 out of 182 countries, based on 2007 data, in UNDP 2009 Human Development Report). In the process, Timor-Leste has made an important transition from a low level of human development to a medium level of human development. Second, the recent MEASURE Demographic and Health Surveys (DHS) 2009-10 project — funded by the US Agency for International Development (USAID) - reveals a significantly more positive assessment on key social and human development indicators.

These two positive assessments further encourage the authorities' revealed determination to continue the implementation of a very ambitious reform agenda in order to develop the country and profit both the current and future generations, and reveals that this strategy is already paying off. Good results only mean that it is possible to reach the end of the path faster. The recent achievements in poverty reduction and growth are very encouraging, but there is still a challenging path ahead, and, therefore, the authorities of Timor-Leste invite the international community's involvement in the current strong momentum. 